\title{
Naturalis
}

Epidemiología de las parasitosis transmitidas por moluscos en el NE de Argentina : Puerto Iguazú sitio de intercambio en la triple frontera

\section{Valente, Romina}

Doctor en Ciencias Naturales

Dirección: Navone, Graciela T.

Co-dirección: Díaz, Julia I.

Facultad de Ciencias Naturales y Museo

2017

Acceso en:

http://naturalis.fcnym.unlp.edu.ar/id/20180418001574

\section{(c) (i) (2) \\ BY NC SA}

Esta obra está bajo una Licencia Creative Commons

Atribución-NoComercial-Compartirlgual 4.0 Internacional

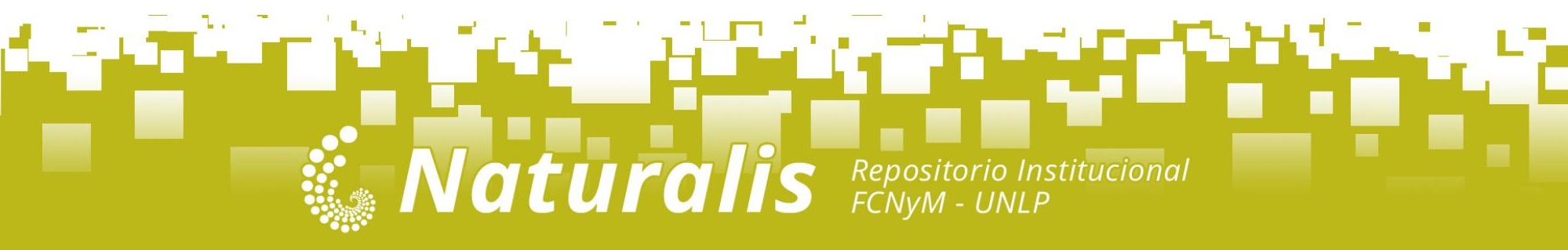




\section{EPIDEMIOLOGÍA DE LAS PARASITOSIS TRANSMITIDAS POR MOLUSCOS EN EL NORESTE DE ARGENTINA: PUERTO IGUAZÚ SITIO DE INTERCAMBIO EN LA TRIPLE FRONTERA}

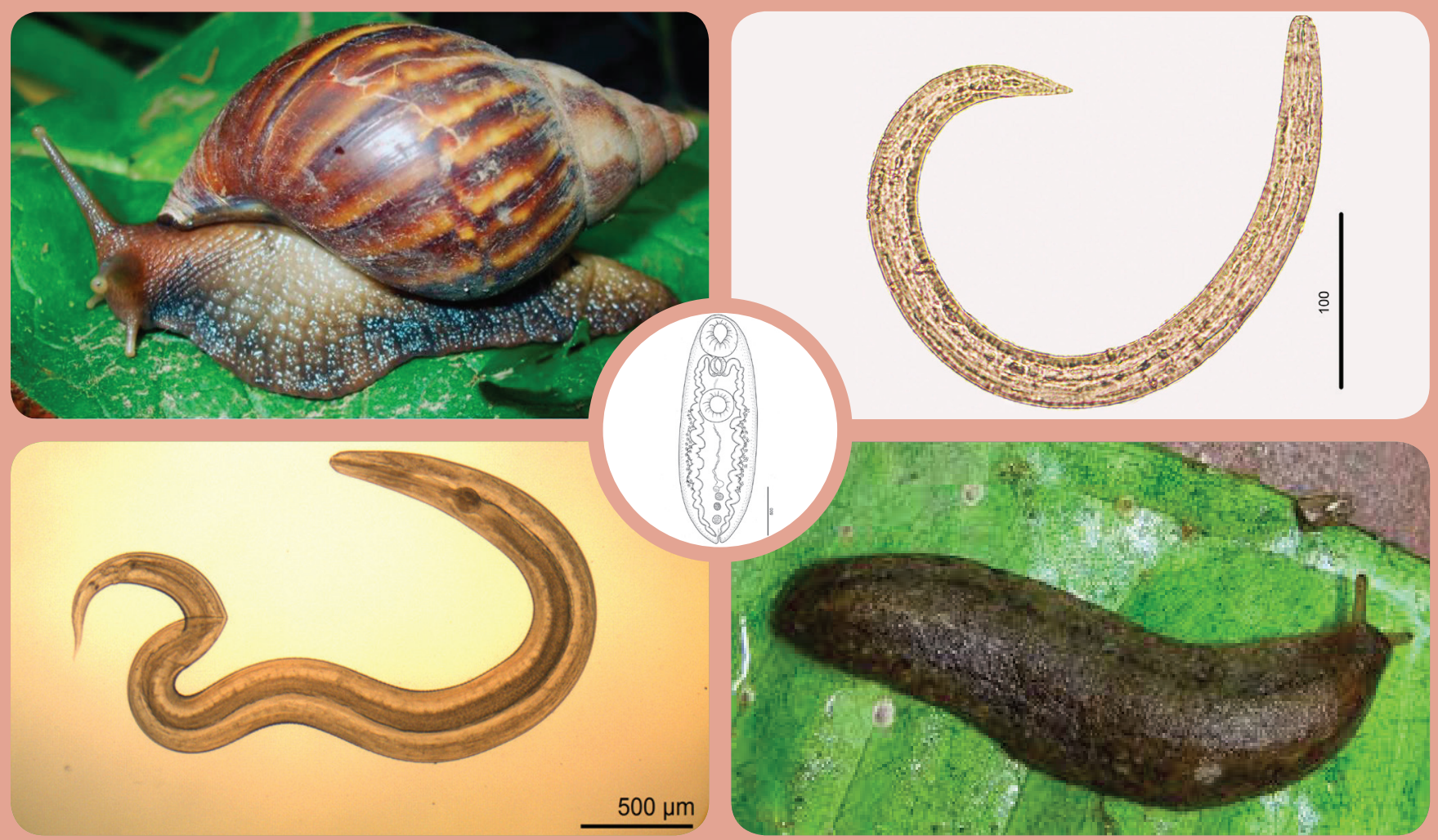

Tesis Doctoral

\section{LIC. ROMINA VALENTE}

DIRECTORAS:

DRA. GRACIELA T. NAVONE DRA. JULIA I. DIAZ

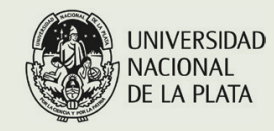


A mi papas, por enseñarme a luchar por mis sueños, demostrándome que con honestidad, sacrificio y paciencia todo se puede.

A mis hermanos Gimena y Pablo por creer en mí. Porque nunca me permitieron bajar los brazos. Gracias por calmar los momentos de angustia y desesperación.

A mis sobrinos que son el motor de mi vida.

A Sergio, por su Amor. 
* Al Consejo Nacional de Investigaciones Científicas y Técnicas (CONICET) por el otorgamiento de la Beca Doctoral con la cual se realizó esta tesis.

* Al Consejo Nacional de Investigaciones Científicas y Técnicas (CONICET), la Agencia Nacional de Promoción Científica y Tecnológica (ANPCyT) y la Universidad Nacional de La Plata (UNLP) por el otorgamiento de subsidios que facilitaron la realización de este trabajo.

* Al Instituto Nacional de Medicina Tropical (INMeT) y al Centro de Estudio Parasitológicos y de Vectores (CEPAVE), por brindarme el espacio y la infraestructura donde desarrollar este trabajo.

* A Graciela por haberme acompañado, apoyado y estimulado a seguir creciendo. Me enseñaste amar esta profesión y a seguir luchando a pesar de los obstáculos. Me demostraste que trabajando con honestidad, empeño y alegría todo tiene su fruto. Gracias por tus consejos, y por todas las palabras de aliento.

* A Julia por abrirme las puertas del Laboratorio cuando más lo necesitaba, enseñandome a dar mis primero pasos en este camino. Por aconsejarme y guiarme. Por brindarme la tranquilidad, seguridad y confianza para seguir adelante. Por darme esas palabras de aliento para no caer. Gracias por estar siempre. Gracias a las dos por su dedicación, confianza y libertad.

* A Daniel por haberme abierto las puertas del Instituto.

* A mis amigas del INMeT Romi y Angie que hicieron mi estadía en Iguazú más amena.

* A mis compañeras/os del CEPAVE: Guille, Ceci E, Agus, Tati, Cailo, Ro, Brunito, Naty, Sofi, Eze, Eli, Bruno, Pao, Andre S, Andre F, Vicky, Lore, Ceci C, Julisan y Marie por su aliento incondicional.

* A mi amiga Pao por esas lindas charlas entre mates, por sus palabras de apoyo y contención.

* A Nilso, Martín, Emilio, Popy, Sole C, Marisol, Jorge, Rodrigo y Sergio Miquel porque en algún momento dedicaron su tiempo en ayudarme. Laura gracias por la hermosa portada. Graciela agradezco tu dedicación y esfuerzo en enseñarme a comprender la estadística. Gracias Kabe por los dibujos.

* A Romi y al Carly por refugiarme en su hogar cada vez que me sentía sola. Fueron mi contención, mi apoyo, y supieron aconsejarme cuando más lo necesite. Romi, gracias por todo, y principalmente por tu amistad.

* A mis amigos que conforman diferentes grupos: Lory, Mary, Vero, Solana, Solange, Marie, Sole, Ro, Naty, Gise, Naty C, Celes, Vale, Yami y Debo.

* A mi amada familia Leguiza por ser otro sostén importante en mi vida. Sus palabras, sus abrazos y su amor me dieron la familia que había perdido. Gracias Irma por ser mi mamá del corazón. 
* A mi hermana de la vida Legui, que me sostuvo en el peor momento de mi vida, y no me dejo caer nunca. Festejaste mis alegrías, y seguís acompañándome incondicionalmente. Gracias por tu fiel y eterna amistad. A Marian, Tizi y Asti por demostrarme tanto amor y por compartir tantos momentos hermosos.

* A mis hermanos Gimena y Pablo que me sostuvieron y contuvieron siempre. Nunca me dejaron rendirme. Sus consejos y palabras permitieron que logre llegar hasta acá.

* A mi cuñada Ceci con la que cuento siempre. A mis sobrinos Isa, Mateo, Cami y Emma que son los amores de mi vida.

A Sergio, por amarme pacientemente, por aparecer en mi vida cuando menos lo esperaba pero cuando más lo necesitaba. A Sofi y Valen por su cariño.

* Y a MI PAPÁ. Espero que sea donde estés te sientas orgulloso de la persona en que me convertí. Estas en mi corazón siempre. Te amo infinitivamente.

Muy especialmente quiero agradecer a todas las personas de la ciudad de Puerto Iguazú por abrirme las puertas de su casa desinteresadamente, y siempre con una sonrisa, sin ellos no podría haber realizado este trabajo. La portada se la dedico a ellos con el rojo de la tierra colorada y el verde de la selva Misionera 
Los gasterópodos pueden actuar como hospedadores intermediarios de ciclos de nematodes Metastrongyloidea (Strongylida) de importancia en sanidad humana y animal. Los metastrongylidos incluyen cuatro especies de importancia sanitaria a nivel mundial: Angiostrongylus cantonensis, Angiostrongylus costaricensis, Angiostrongylus vasorum y Aelurostrongylus abstrusus.

Lissachatina fulica fue detectado en la ciudad de Puerto Iguazú, Misiones en el año 2010 y es una especie que está en simpatría con las especies nativas Phyllocaulis variegatus y Latipes erinaceus. En el Noreste Argentino (NEA) aún no existen estudios parasitológicos en gasterópodos terrestres. El objetivo de la presente investigación fue evaluar la distribución, dispersión y parasitofauna del caracol gigante africano L. fulica, desde su detección hasta la actualidad y de las babosas nativas con el fin de determinar su rol en la transmisión de helmintos parásitos, con especial énfasis en los nematodes metastrongylidos.

Se analizaron un total de 995 ejemplares de L. fulica, 120 de P. variegatus y 14 de L. erinaceus, colectados en el área urbana de Puerto Iguazú los cuales fueron medidos para posteriormente ser clasificados en cuatro intervalos de tallas. Los hospedadores fueron procesados utilizando dos técnicas diferentes: evisceración y digestión artificial.

Se identificaron tres especies de helmintos parásitos: Brachylaima sp. (Trematoda, Brachylaimidae), Strongyluris sp. (Nematoda, Heterakidae) y Aelurostrongylus abstrusus (Nematoda, Angiostrongylidae). A pesar de los bajos valores de prevalencia, abundancia media e intensidad media hallados, los resultados mostraron una relación significativamente positiva entre la carga parasitaria y el tamaño de los hospedadores. Así mismo, se observó que las variables ambientales no influyen sobre la distribución de las especies parásitas.

El análisis de distribución espacial de L. fulica desde su detección en la Argentina hasta la actualidad se incrementó, encontrándose así nuevas áreas focos dentro de la ciudad de Puerto Iguazú. En contraparte la distribución de P. variegatus y L. erinaceus disminuyó a lo largo del tiempo de muestreo.

Los mapas predictivos de distribución de nicho ecológico muestran a futuro un cambio radical en la distribución espacial de L. fulica, alcanzando una expansión hacia nuevas áreas geográficas actualmente insospechadas. Se observó que las áreas más 
comprometidas para el futuro son el este, oeste y sur de Sudamérica, incluida casi la totalidad de la de Argentina.

Por otra parte se observó que los factores ambientales influyen en el tamaño corporal de L. fulica, observándose una significativa asociación entre las tallas más pequeñas $[0-3 \mathrm{~cm}$ ) y períodos con escasas precipitaciones-bajas temperaturas (período de hibernación), y entre las tallas más grandes [6-12 cm) y períodos con temperaturas promedio de 20-25 $\mathrm{C}^{\circ}$, y precipitaciones continuas (períodos postestivación). En relación a las babosas Veronicellidae las tallas intermedias [3-9 cm) fueron las más abundantes en el bimestre octubre-noviembre, período en el que se observa un incremento en las lluvias y descenso de las temperaturas, aspecto importante para organismos desnudos que respiran a través de su tegumento.

La continuidad de estos estudios sobre la parasitofauna de gasterópodos terrestres y en particular de L. fulica permitirá conocer su rol en la transmisión de parásitos de importancia sanitaria en Puerto Iguazú y en las áreas de expansión de distribución prevista para los próximos años, dado que representan un escenario epidemiológico preocupante. 
The gastropods can act as intermediate hosts of cycles of Metastrongyloidea nematodes (Strongylida) of importance in human and animal health. Metastrongylid includes four species of sanitary importance worldwide: Angiostrongylus cantonensis, Angiostrongylus costaricensis, Angiostrongylus vasorum and Aelurostrongylus abstrusus.

Lissachatina fulica was detected in 2010 in the Puerto Iguazú city, Misiones. It is a mollusc species sympatric with the native species $P$. variegatus and $L$. erinaceus. In the Northeast of Argentina (NEA) there are no parasitological studies on terrestrial gastropods. The aim of the present investigation was to evaluate the distribution, dispersion and parasitofauna of the giant African snail L. fulica, from its detection in Argentina to the present, and the sympatric native slugs, in order to determine their role in the transmission of parasitic helminths, with special emphasis on the metastrongylid nematodes.

A total of 995 specimens of L. fulica, 120 of P. variegatus and 14 of L. erinaceus were collected in the urban area of Puerto Iguazú. These hosts were measured, clasificated into 4 size intervals, and processed using two different techniques: evisceration and artificial digestion.

Three species of parasitic helminths were identified: Brachylaima sp. (Trematoda, Brachylaimidae), Strongyluris sp. (Nematoda, Heterachidae) and Aelurostrongylus abstrusus (Nematoda, Angyostrongilidae). Despite the low values of prevalence, mean abundance and mean intensity found, the results showed a significantly positive relationship between size of the hosts and parasitic parameters. Likewise, it was observed that environmental variables do not influence the distribution of parasitic species.

The spatial distribution of L. fulica from its detection in the Argentina to the present time increased, finding new focus areas inside Puerto Iguazú city. In contrast, the distribution of $P$. variegatus and $L$. erinaceus decreased over the time of sampling. 
The predictive maps of ecological niche distribution show a radical change in the spatial distribution of L. fulica in the future, reaching an expansion towards new geographical areas currently unsuspected. It was observed that the most committed areas for the future are the east, west and south of South America, with almost all of Argentina.

On the other hand, it was observed that environmental factors influence the body size of L. fulica, observing a significant association between smaller sizes [0-3 cm) and periods with low rainfall-low temperatures (hibernation period), and between the largest sizes $\left[6-12 \mathrm{~cm}\right.$ ) and periods with average temperatures of $20-25 \mathrm{C}^{\circ}$, and continuous rainfall (post-test period). In relation to slugs Veronicellidae the intermediate sizes [3-9 $\mathrm{cm}$ ) were the most abundant in October-November, period in which an increase in rainfall and decrease in temperatures is observed, an important aspect for naked organisms that breathe through his tegument.

The continuousness of these studies on the parasitic fauna of terrestrial gastropods, and in particular of L. fulica, will allow us to know their role in the transmission of parasites of sanitary importance in Puerto Iguazú and in the areas of distribution expansion expected for the coming years, since they represent a worrisome epidemiological scenario. 


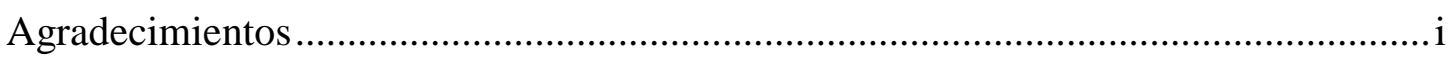

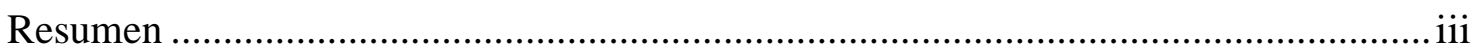

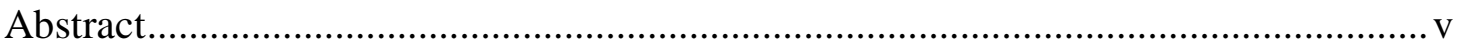

\section{CAPÍTULO 1: INTRODUCCIÓN GENERAL}

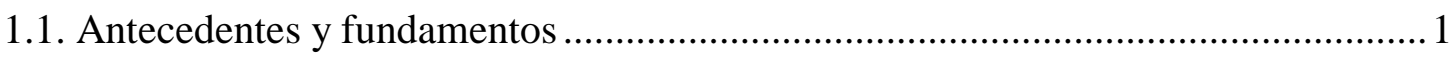

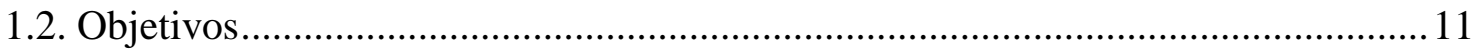

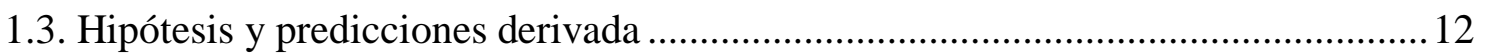

\section{CAPÍTULO 2: METODOLOGÍA}

2.1. Caracterización del área de estudio ................................................................. 13

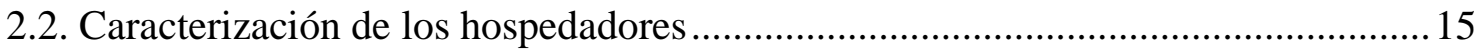

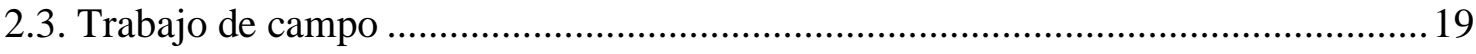

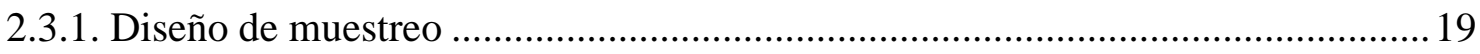

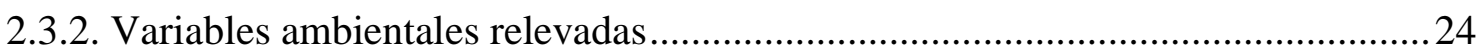

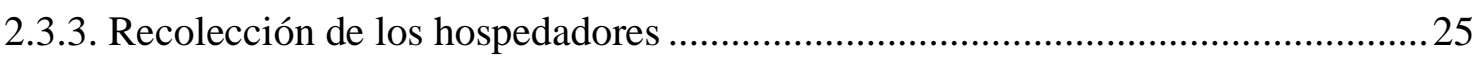

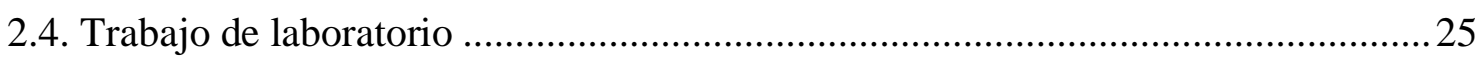

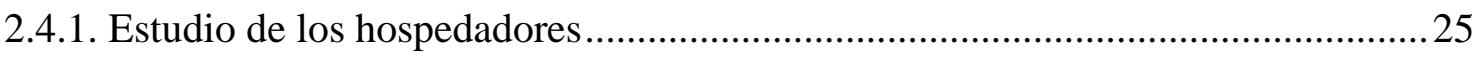

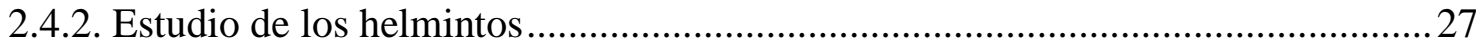

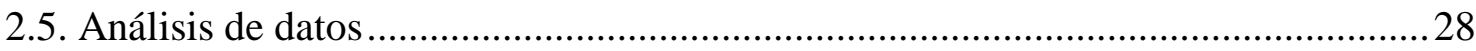

\section{CAPITULO 3: RESULTADOS Y DISCUSIÓN}

\section{Parte I: Los hospedadores}

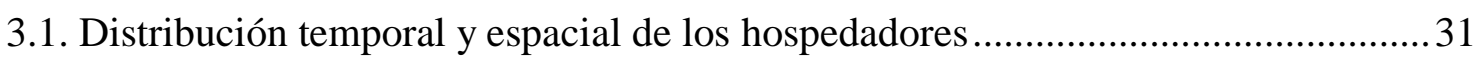

3.2. Distribución de tallas de los hospedadores en función del tiempo y las variables

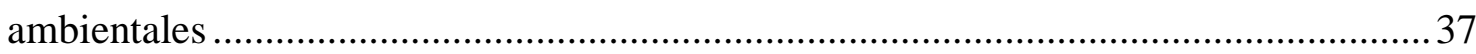

3.3. Mapas predictivos de distribución de nichos ecológicos de L. fulica ...................... 44

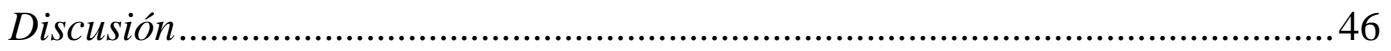




\section{Parte II: Los parásitos}

3.4. Identificación taxonómica de los helmintos 51

Comentarios acerca de los resultados obtenidos a partir de las metodologías

aplicadas..... 59

3.5. Parámetros poblacionales y su relación con las tallas de los hospedadores 60

3.6. Influencia de los componentes temporal y ambiental en la distribución parasitaria. 68

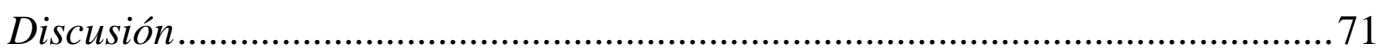

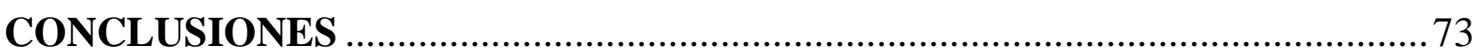

ANEXOS

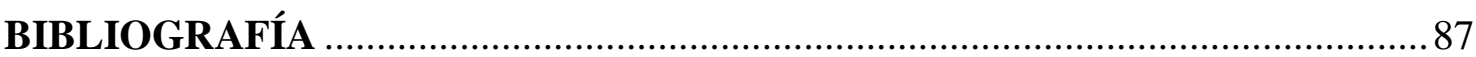




\section{Introducción General}

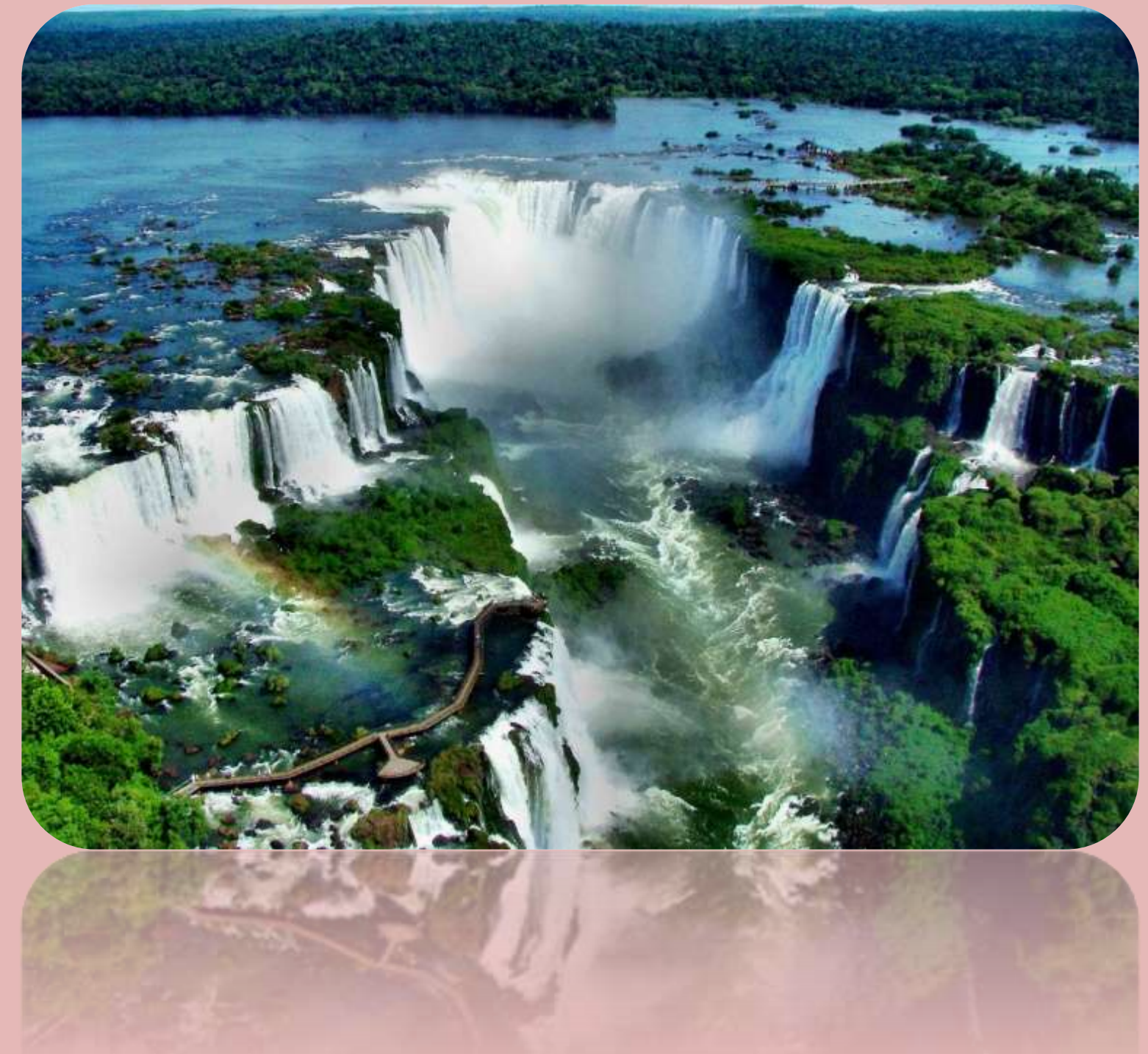




\section{INTRODUCCION GENERAL}

\subsection{ANTECEDENTES Y FUNDAMENTOS}

El parasitismo es un tipo de relación simbiótica en la cual uno de los integrantes (el parásito) presenta una dependencia metabólica con el otro (el hospedador) que determina la obligatoriedad de su relación (Wisnivesky, 2003). En esta interacción interespecífica, puede ocurrir que coexistan varias especies parásitas en una población hospedadora, pero ¿cuál o cuáles serán los determinantes que permitan la expansión o extinción de una especie parásita en una población hospedadora? La teoría epidemiológica proporciona un marco para abordar estas cuestiones (Poulin \& Morand, 2004).

Los modelos epidemiológicos generalmente asumen que el hospedador está biológicamente preparado para tolerar este vínculo, permitiendo que las poblaciones parásitas sobrevivan y se reproduzcan dentro de una población hospedadora. Es importante considerar el intercambio de hospedadores, donde los parásitos "saltan" a otros hospedadores que tienen una estrecha relación filogenética. Teniendo en cuenta estas características, los modelos epidemiológicos intentan explicar la dinámica de las transmisiones parasitarias (Poulin \& Morand, 2004).

En este contexto, la complejidad de la dinámica de las poblaciones parásitas puede describirse a partir del análisis de ciertas características epidemiológicas tales como la tasa de dispersión de los parásitos en sus poblaciones hospedadoras, el número de hospedadores requeridos para que el parásito logre una estabilidad poblacional, y el nivel promedio de infestaciones parasitarias en las poblaciones hospedadoras (Anderson \& May, 1979; May \& Anderson, 1979).

A la interacción entre el hospedador, el agente infeccioso y el ambiente, se la conoce como triada epidemiológica. Esta interacción a veces involucra a un vector $\mathrm{u}$ hospedador intermediario, organismo capaz de portar y transmitir un agente infeccioso, y muchas veces puede culminar en enfermedad. Aquellos factores que pueden afectar la triada epidemiológica están vinculados a las características del hospedador, al grado de infectividad del agente patógeno y los factores ambientales (Ortiz et al., 2004). De este modo, en el proceso de transmisión parasitaria una especie parásita pasa de un hospedador a otro para asegurar su dispersión y facilitar su reproducción, con 
eventuales adaptaciones fisiológicas que aumentan la probabilidad de transmisión (Wisnivesky, 2003).

En este marco es importante comprender el concepto de ecoepidemiología (Ortiz et al., 2004), que se define como el estudio de los determinantes de la dinámica de transmisión, distribución y abundancia de patógenos en sistemas naturales/silvestres, y de su impacto sobre las poblacionales hospedadoras, permitiendo entender la influencia del ambiente en la interacción hospedador-parásito. En esta interacción hospedadorparásito se tienen en cuenta aspectos genéticos, biológicos y ecológicos de ambos miembros de la relación (Esch et al., 2002; Bush et al., 2011). Por lo tanto, la ecoepidemiología postula que para analizar las infestaciones a nivel poblacional es necesario abordar el estudio del sistema de interacciones parásito/hospedador/ambiente en su conjunto (Smith et al., 2005).

Si bien el hospedador definitivo (HD) es importante en la expansión de la infección debido a su mayor capacidad de trasladarse (Haseeb \& Fried, 1997) y porque en él ocurre la maduración y reproducción sexual del parásito, el hospedador intermediario juega un rol fundamental en el ciclo de transmisión, porque en muchos casos el parásito se multiplica en él y aumenta en forma exponencial el número de larvas. Entre los nematodes, algunas especies tienen larvas de vida libre que buscan activa o pasivamente a sus hospedadores. En este caso la dispersión se realiza cuando las larvas penetran activamente o son consumidos por el HI, en el cual se desarrolla el estadio infectivo (Wisnivesky, 2003), el cual luego ingresa al HD a través de la dieta o activamente. En este marco, resulta esencial conocer la biología de los hospedadores definitivos e intermediarios, para poder interpretar los mecanismos que tienen lugar en la transmisión parásito/hospedador/ambiente.

Teniendo en cuenta lo expuesto, es importante el estudio de los moluscos gasterópodos (caracoles y babosas), ya que resultan ser hospedadores intermediarios de parásitos que afectan la salud humana y animal, tales como algunos nematodes (e.g. Metastrongylidae) y trematodes (e.g. Schistosomatidae, Fasciolidae) (Thiengo \& Amato, 1995; Marquardt et al., 2000; Borda et al., 2006; Ohlweiler et al., 2010; Virgillito, 2012), los cuales se distribuyen ampliamente en América del Sur. 
Rol de Lissachatina fulica y Veronicellidae como hospedadores intermediarios de helmintos de importancia sanitaria en Argentina

Los endoparásitos están expuestos indirectamente a condiciones externas por un período de tiempo en el que permanecen dentro del hospedador. Factores extrínsecos como la temperatura pueden tener un impacto en ellos, modificando la fisiología de los hospedadores, y causando un efecto indirecto sobre los parásitos. Esta situación es más perjudicial cuando el hospedador no posee una regulación interna (efecto buffer) de las condiciones externas, como es el caso de los hospedadores invertebrados (Poulin, 2007).

Entre los hospedadores invertebrados, algunas especies de caracoles pueden actuar como hospedadores intermediarios de nematodes de importancia en sanidad humana y animal, entre las que se encuentra el "caracol gigante africano" Lissachatina fulica (Férrusac, 1821) (= Achatina fulica) (Carvalho et al., 2003; Thiengo et al., 2010). Esta especie pertenece a la familia Achatinidae (Mollusca: Stylommatophora), un grupo de caracoles terrestres nativos de África, en particular Sub-Sahara (países del continente africano que no limitan con el Mar Mediterráneo) (Raut \& Barker, 2002). La dispersión de $L$. fulica alrededor del mundo comenzó a principios del siglo XIX expandiéndose hacia la Costa Este de África, posteriormente a Madagascar, Mauritius y Seychelles Este molusco ingresó a la India en 1847, expandiéndose luego a casi todo el continente asiático, llegando a Taiwán, Malasia, Japón, Sumatra y Filipinas a fines de 1930 (Cross, 2007).

Lissachatina fulica fue introducido en Hawaii en 1936, por una pobladora que provenía de Japón. Posteriormente se expandió en las Islas Pacíficas entre las décadas del '70 al '90 (Alicata, 1991).

Lissacathina fulica fue introducido en el continente americano, Florida (USA), en 1966 desde Hawaii y fue reportado oficialmente en 1984 (Duffy et al., 2004). A fines de 1988 se estableció en la Isla Martinica, para luego introducirse en Barbados y Santa Lucia (islas del Caribe). Posteriormente fue introducido en Brasil, a principio de 1980, pero no fue reportado hasta 1997, el mismo año en que se registró en Venezuela. Entre 2005 y 2013 se notificó su presencia en Ecuador, Colombia, Galápagos, Bolivia y Paraguay (Thiengo et al., 2007; Vogler et al., 2013) (Figura 1) (Tabla 1).

En el año 2010 se registró por primera vez a L. fulica en la Argentina en la ciudad de Puerto Iguazú, Misiones (Gutiérrez Gregoric et al., 2011). Posteriormente, se 
observó en la ciudad de Corrientes (Diaz et al., 2013; Gutiérrez Gregoric et al., 2013). En muchos países el propósito de su introducción fue su utilización como carnada para pesca o para consumo personal en reemplazo del Cornu aspersum Müller, 1774. A escala mundial L. fulica constituye una de las 100 especies invasoras más peligrosas del mundo (Lowe et al., 2004).

El éxito de L. fulica se debe a su alta tasa de reproducción que ocasiona un crecimiento poblacional explosivo, otorgándole un potencial invasor altamente desarrollado y pudiendo constituir una verdadera "plaga". Los factores ambientales determinan su crecimiento corporal y biomasa. Es así que las condiciones climáticas pueden inducir a períodos de letargo, principalmente si la temperatura y la humedad alcanzan valores bajos. Una vez que transcurre este lapso de tiempo, pueden aparecer cambios fisiológicos en L. fulica que afectan su desarrollo (Albuquerque et al., 2007). Además posee diversas estrategias de protección y capacidad adaptativa frente a condiciones ambientales desfavorables. Esta situación se ve favorecida por el aumento desproporcionado de alimento y/o por la falta de depredadores que actúen como agentes naturales de control. En áreas tropicales y subtropicales L. fulica ocasiona daños considerables en los sistemas agrícolas ya que es un herbívoro/polífago que ataca gran variedad de cultivos, entre los cuales se encuentran de cacao, maíz, algodón, cambur, cítricos, fruta de pan, lechuga, melón, piña (Matinella et al., 2009).

Otras especies de gasterópodos que actúan como hospedadores intermediarios de nematodes de importancia en sanidad humana y animal, son las babosas de la familia Veronicellidae (Mollusca: Systelommatophora). Estos son gasterópodos estrictamente terrestres, que se distribuyen en áreas tropicales y subtropicales. De los 23 géneros distribuidos mundialmente, cinco se encuentran en la Argentina: Angustipes, Phyllocaulis, Latipes, Heterovaginina y Saranisula (Santin \& Miquel, 2015). Las especies de Veronicelidos que se encuentran en el Noreste Argentino son cinco: Angustipes difficilis (Colosi, 1921), Phyllocaullis soleiformis (Orbigny, 1935), Phyllocaulis variegatus (Semper, 1885), Latipes erinaceus (Colosi, 1921) y Saranisula linguaeformis (Semper, 1885) (Santin \& Miquel, 2015). 


\section{$\underline{\text { Referencias }}$}

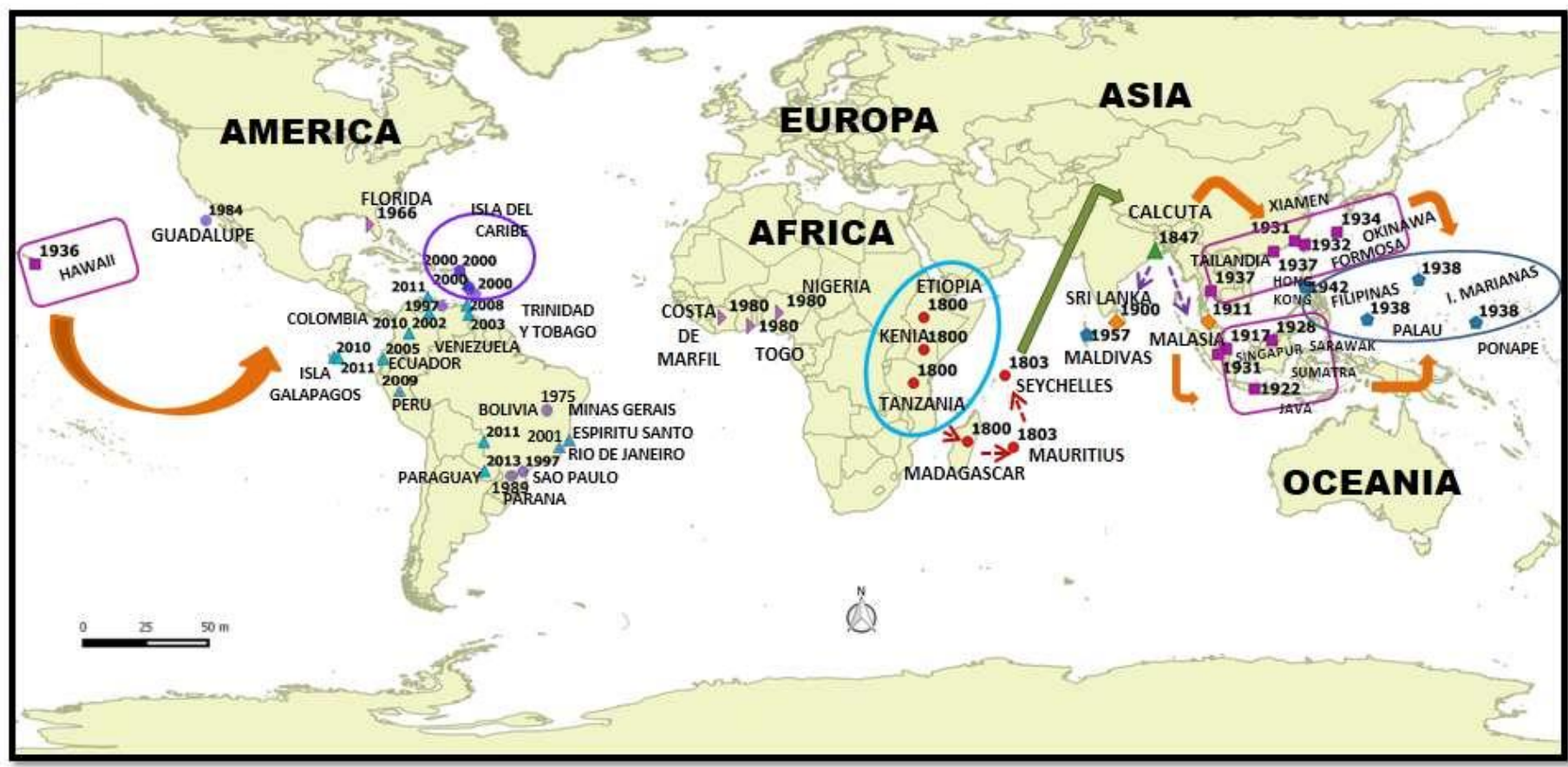

Períodos de colonización de L. fulica en el mundo

- 1800-1803

1847

- 1900-1911

1917-1937

1938-1957

1966-1980

1984-2000

- 2001-2013

Figura 1. Distribución mundial de Lissachatina fulica desde 1800 hasta 2013. 
Tabla 1. Distribución actual de Lissachatina fulica

\begin{tabular}{|c|c|c|c|}
\hline $\begin{array}{l}\text { Año del } \\
\text { primer } \\
\text { registro }\end{array}$ & Sitio geográfico & Continente & Literatura consultada \\
\hline 1800 & $\begin{array}{c}\text { Kenia; Etiopía; Tanzania; } \\
\text { Madagascar }\end{array}$ & África & Mead, 1961 \\
\hline 1803 & Mauritius, Seychelles & África & Mead, 1961 \\
\hline 1847 & Calcuta & Asia & Mead, 1961 \\
\hline 1900 & Sri Lanka & Asia & Mead, 1961 \\
\hline 1911 & Península de Malasia & Asia & Mead, 1961 \\
\hline 1917 & Singapur & Asia & Mead, 1961 \\
\hline 1922 & Java & Asia & Mead, 1961 \\
\hline 1928 & Borneo, Malasia & Asia & Mead, 1961 \\
\hline 1931 & $\begin{array}{l}\text { Xiamen, China; } \\
\text { Sumatra }\end{array}$ & Asia & Mead, 1961 \\
\hline 1932 & Formosa, Taiwán & Asia & Mead, 1961 \\
\hline 1934 & Okinawa & Asia & Mead, 1961 \\
\hline 1936 & Hawaii, USA & América & Mead, 1961 \\
\hline 1937 & Tailandia & Asia & Mead, 1961 \\
\hline 1937 & Hong Kong, China & Asia & Mead, 1961 \\
\hline 1938 & Ponape & Asia & Mead, 1961 \\
\hline 1938 & Palau & Oceanía & Mead, 1961 \\
\hline 1938 & Islas Marianas, USA & América & Mead, 1961 \\
\hline 1942 & Filipinas & Asia & Mead, 1961 \\
\hline 1957 & Maldivas & Asia & Mead, 1961 \\
\hline 1966 & Florida, USA & América & $\begin{array}{c}\text { Mead, } 1961 \\
\text { Duffy et al.2004 }\end{array}$ \\
\hline 1975 & Minas Gerais & América & Zanol et al. 2010 \\
\hline 1980 & $\begin{array}{l}\text { Nigeria; Togo; } \\
\text { Costa de Marfil }\end{array}$ & África & Raut \& Barker, 2002 \\
\hline 1984 & Guadalupe & América & Ciomperlik et al. 2013 \\
\hline 1989 & Paraná, Brasil & América & Teles \& Fontes, 2002 \\
\hline 1988 & Isla Martinica, Mar Caribe & América & Ciomperlik et al. 2013 \\
\hline 1990 & Samoa, Tailandia & Asia & Ciomperlik et al. 2013 \\
\hline 1997 & São Paulo & América & Thiengo et al. 2007 \\
\hline 1997 & Caracas, Venezuela & América & Ciomperlik et al. 2013 \\
\hline 2000 & $\begin{array}{c}\text { Barbados/Santa Lucia/Anguilla, } \\
\text { Mar Caribe }\end{array}$ & América & Ciomperlik et al. 2013 \\
\hline 2001 & Rio Janeiro, Espiritu Santo & América & Thiengo et al. 2007 \\
\hline 2002 & Guanare, Venezuela & América & Martínez-Escarbassiere et al. 2008 \\
\hline 2003 & Delta Amacuro, Venezuela & América & Martínez-Escarbassiere et al. 2008 \\
\hline 2005 & Esmeralda, Ecuador & América & Ciomperlik et al. 2013 \\
\hline 2007 & República Dominicana & América & Ciomperlik et al. 2013 \\
\hline 2008 & Trinidad y Tobago & América & Ciomperlik et al. 2013 \\
\hline 2009 & Perú & América & Borrero et al. 2009 \\
\hline 2010 & $\begin{array}{l}\text { Isla Santa Cruz, Ecuador; } \\
\text { Colombia; Argentina }\end{array}$ & América & $\begin{array}{c}\text { Borrero et al. } 2009 \\
\text { Gutiérrez Gregoric et al. 2011, } \\
2013\end{array}$ \\
\hline 2011 & Isla San Cristóbal, Ecuador & América & Borrero et al. 2009 \\
\hline 2011 & Aruba, Mar Caribe & América & Ciomperlik et al. 2013 \\
\hline 2011 & Puerto Suarez, Bolivia & América & Correoso \& Coello, 2009 \\
\hline 2013 & Misiones, Paraguay & América & Ciomperlik et al. 2013 \\
\hline
\end{tabular}




\section{Nematodes Metastrongyloidea}

Los nematodes parásitos de la superfamilia Metastrongyloidea poseen gran importancia en sanidad humana y animal, ya que utilizan como hospedadores definitivos a mamíferos, pudiendo afectar al hombre y animales domésticos. Los ejemplares de la familia Angiostrongylidae utiliza en sus ciclos de vida a gasterópodos terrestres tales como L. fulica, babosas Veronicellidae, Subulina octona (Bruguière, 1798) y Bradybaena similaris (Férussac, 1821) (Dorta-Contreras et al., 2007; Sabina Molina et al., 2009). Los individuos de la familia Angiostrongylidae tienen como hospedadores definitivos silvestres a roedores, insectívoros, marsupiales y carnívoros, e incluye cuatro especies de importancia sanitaria: Angiostrongylus cantonensis (Chen, 1935), Angiostrongylus costaricensis Morera y Céspedes, 1971, Angiostrongylus vasorum (Baillet, 1866) y Aelurostrongylus abstrusus (Railliet, 1898) (Anexo I).

\section{Angiostrongylus cantonensis}

Este nematode fue descrito inicialmente como Pulmonema cantonensis (Chen, 1935), a partir de pulmones de ratas infectadas de forma natural (Rattus norvegicus Berkenhout, 1769 y Rattus rattus Linnaeus, 1758) recolectados en Cantón, China en 1935. Esta especie se conoce comúnmente como "gusano pulmonar" debido a que en su etapa adulta se halla en las arterias pulmonares o en el ventrículo derecho del hospedador definitivo que en general son roedores tales como (e.g. Rattus spp.), cánidos y marsupiales (Spratt, 2015, Robles et al., 2016). Cuando las larvas alcanzan accidentalmente al hombre pueden causar meningoencefalitis eosinofílica (ME). Las patologías/sintomatologías asociadas son: inflamación de meninges del encéfalo, formación de granulomas, afección en los nervios craneales produciendo como consecuencia la parálisis de las extremidades superiores, convulsiones, visión doble, etc (Miyazaki, 1991).

La distribución de A. cantonensis estaba limitada a países de Asia como China y Japón, pero a partir de la II Guerra Mundial (1939-1945), ésta comenzó a ampliarse llegando al sudeste asiático, Islas del Pacífico y Australia. A partir de 1950 comenzaron a aparecer casos de ME en distintos países asiáticos, luego en Hawaii, llegando más adelante al continente americano (Cross, 2004, 2007; Ping Wang et al., 2008). 
El primer reporte de ME en América fue en Cuba en 1981, a partir de allí la enfermedad se expandió por diferentes países de América. Una de las hipótesis indicaría que A. cantonensis fue introducido a la isla cubana por roedores del género Rattus provenientes de Asia (Aguiar et al., 1981; Alicata, 1991; Raccurt et al., 2003; Caldeira et al., 2007; Dorta-Contreras et al., 2007; Valente et al., 2018) (Anexo II) (Figuras 2-3).

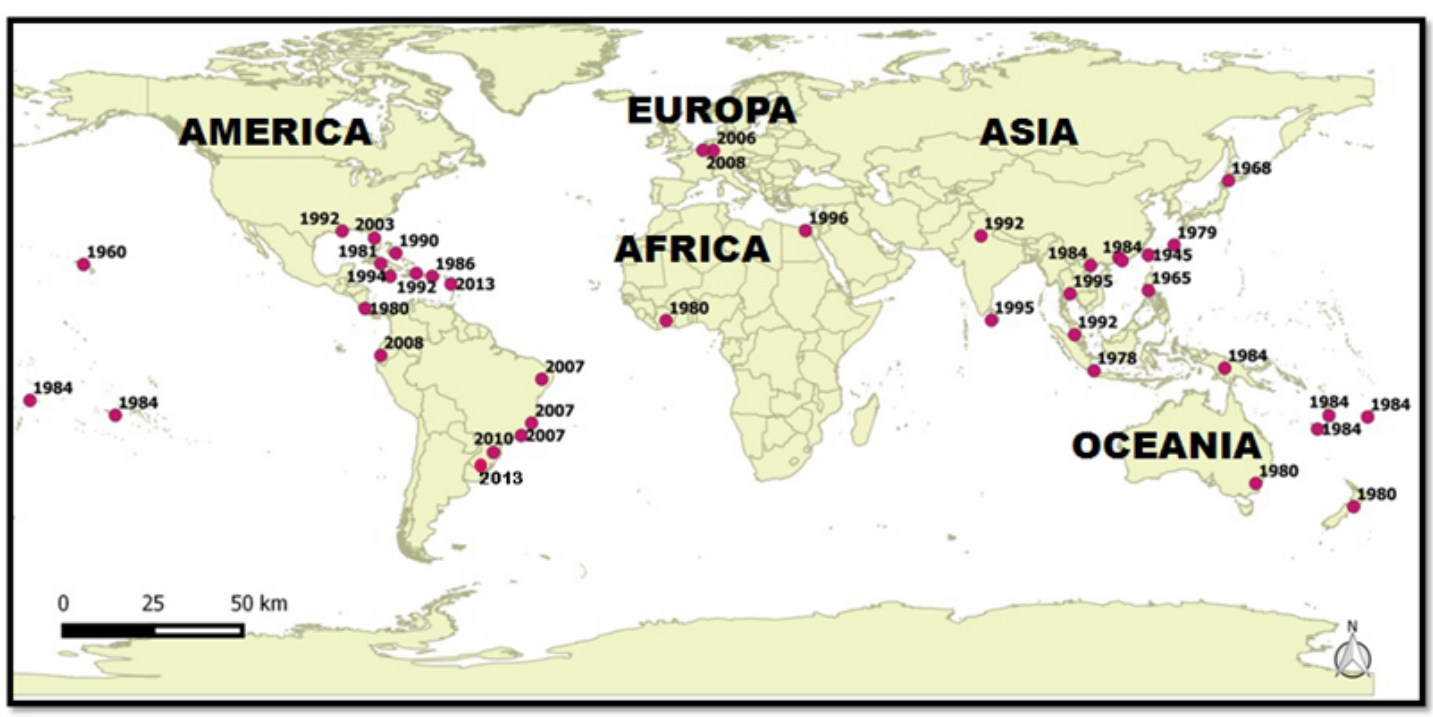

Figura 2. Primeros reportes a nivel mundial de meningoencefalitis eosinofílica transmitida por Angiostrongylus cantonensis.

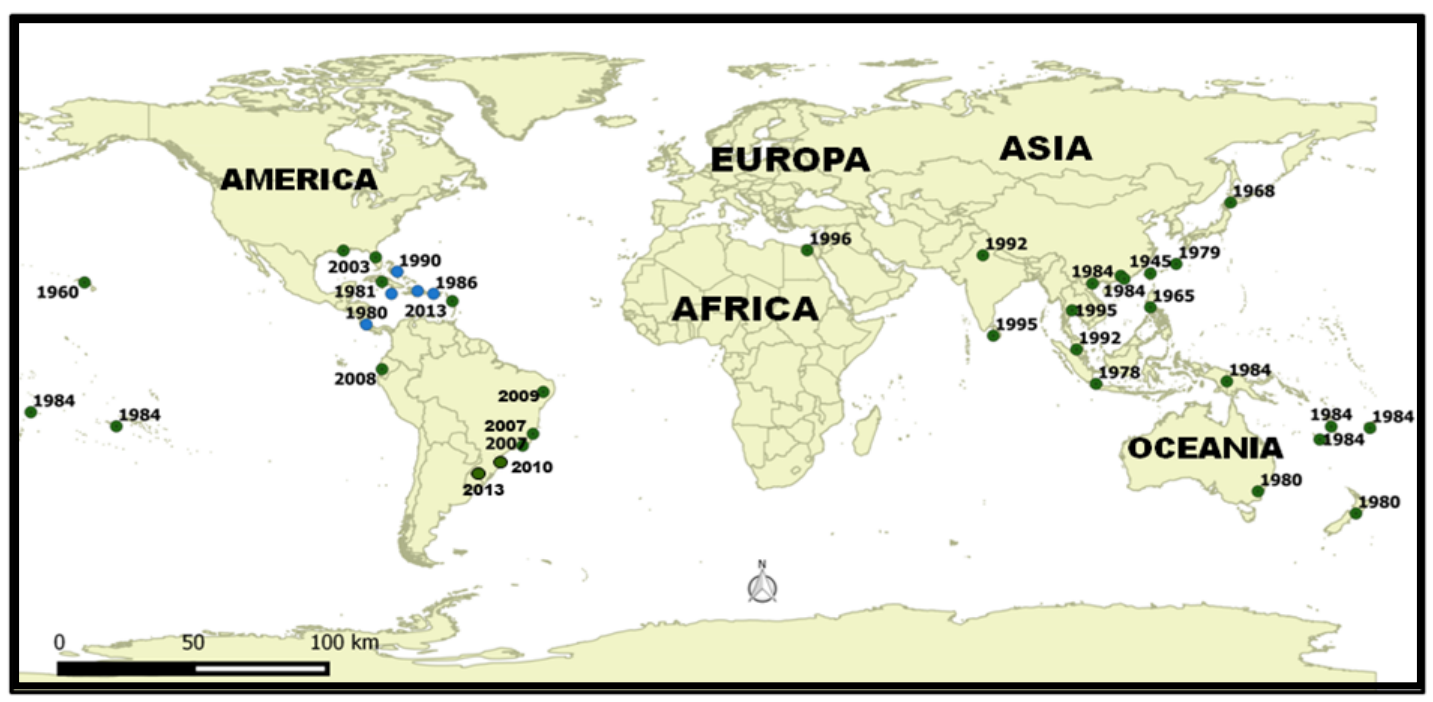

Primeros reportes a nivel mundial de meningoencefalitis eosinofílica transmitida por Angiostrongylus cantonensis, asociada a Lissachatina fulica.

Primeros registros a nivel mundial de meningoencefalitis eosinofílica transmitida por Angiostrongylus cantonensis, no asociada a Lissachatina fulica.

Figura 3. Distribución de meningoencefalitis eosinofílica en el mundo. 


\section{Angiostrongylus costaricensis}

Esta especie es endémica del continente americano y es el agente etiológico de la angiostrongyliasis abdominal (AA). Esta enfermedad fue reportada por primera vez en humanos por Céspedes et al (1967) y Morera (1967) en Costa Rica. Este nematode en su fase adulta se halla en las vías mesentéricas del hospedador definitivo que en general son roedores, cánidos y marsupiales, entre los hospedadores más frecuentes (Robles et al., 2016, Valente et al., 2018). Se distribuye desde los Estados Unidos hasta el Norte de Argentina, con reportes de casos humanos en diferentes países de América (Kaminsky et al., 1995; Maldonado Jr et al., 2012; Valente et al., 2018). Hasta el presente no se han reportado casos fuera del continente americano (Anexo III) (Figura 4).

Las patologías/sintomatologías de AA en el hospedador accidental son: gran dolor abdominal que se asocian a nauseas, formación de tumores a nivel de la región ileocecal, ulceraciones, etc (Miyazaki, 1991).

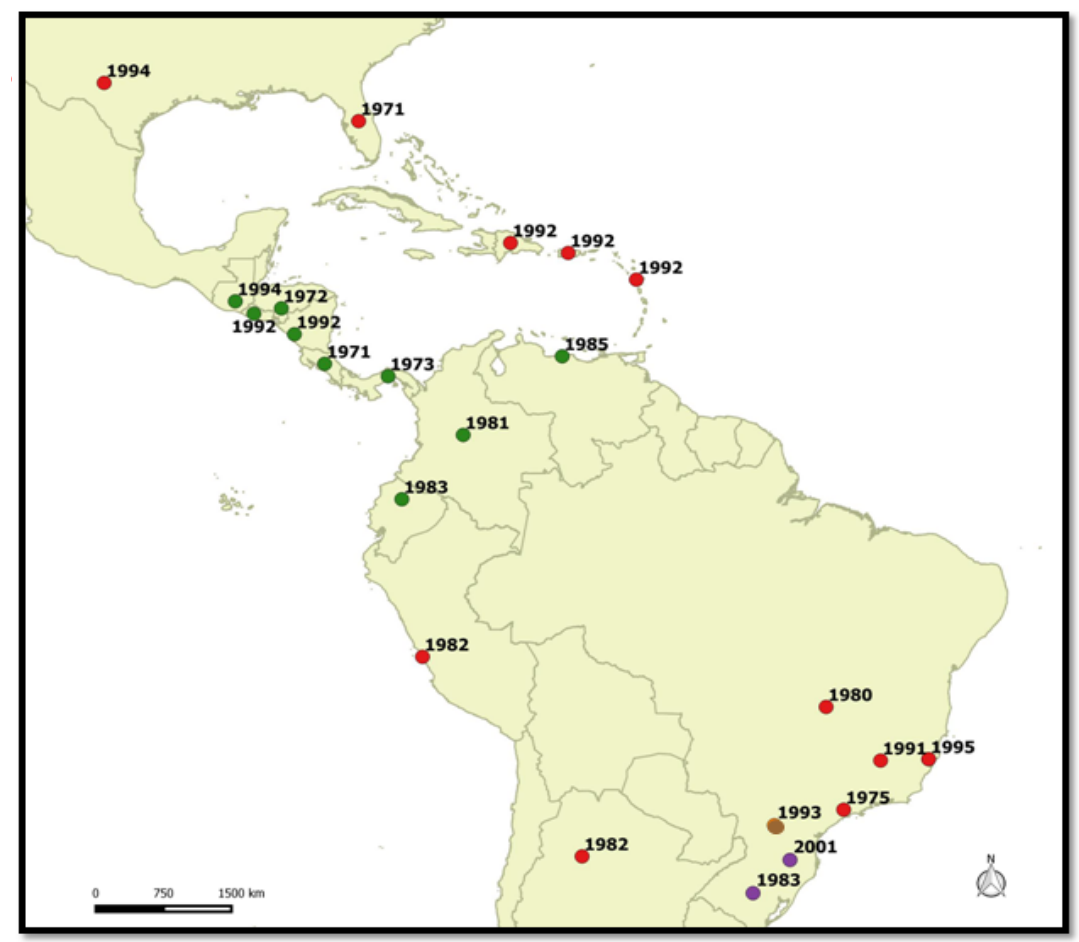

Primeros registros de angiostrongyliasis abdominal en el área endémica asociadas a Veronicellidae (babosas nativas).

Primeros registros de angiostrongyliasis abdominal en el área endémica asociadas a babosas exóticas y nativas.

Primeros registros de angiostrongyliasis abdominal en el área endémica asociadas a babosas exóticas.

Solo registro de enfermedad.

Figura 4. Primeros registros de angiostrongyliasis abdominal en el área endémica transmitido por Angiostrongylus costaricensis. 


\section{Angiostrongylus vasorum}

Se lo halló por primera vez en Francia en el siglo XIX. Debido a que en esa época se lo consideraba una especie endémica se lo llamo "gusano del corazón francés". En la actualidad se lo encuentra en diversos países europeos como Italia, España, Alemania y Portugal (Thiengo et al., 2008; Traversa et al., 2010; Barutzki \& Schaper, 2013). En el continente americano la presencia de esta especie es controversial, debido a que la especie hallada en Brasil (Duarte et al., 2007) sería diferente de la observada en Europa, infiriéndose que la presencia de A. vasorum en América, es el resultado de un antiguo acontecimiento evolutivo que dio lugar a una especiación (Jefferies et al., 2010).

Esta especie de nematode Metastrongyloidea tiene importancia veterinaria debido a que causa diversas patologías a su hospedador definitivos que son los cánidos, produciendo la enfermedad denominada "pulmonía canina". Los síntomas más comunes son: obstrucción respiratoria, trastornos de la coagulación, neumonía e inflamación verminosa, náuseas, tos, disnea y problemas cardiacos (Koch \& Willesen, 2009).

\section{Aelurostrongylus abstrusus}

Tiene una distribución mucho más amplia que A. vasorum, que incluye Francia, España, Alemania, Portugal, Australia, Sudamérica, Norteamérica, y esporádicamente Asia y África (Barutzki \& Schaper, 2013). En América del Sur se lo registró en Brasil y Argentina, con reportes tanto en HI como en HD (Valente et al., 2017). La enfermedad que producen en sus hospedadores definitivos (félidos) se la conoce como "aelurostrongyliasis", también llamada comúnmente neumonía de los gatos. Produce severas patologías tales como bronconeumonía, anorexia, hipertrofia de los alvéolos pulmonares.

En el Noreste Argentino (NEA) se desarrollaron estudios parasitológicos de gasterópodos dulceacuícolas pertenecientes a las familias Planorbidae y Lymnaeidae, hospedadores intermediarios de Trematodes, agentes etiológicos de la esquistosomiasis y fasciolasis (Rumi et al., 2008; Prepelitchi, 2009) pero aún no existen estudios en gasterópodos terrestres del NEA. Teniendo en cuenta las particularidades biológicas y ecológicas de los Achatinidae en el continente sudamericano y la capacidad de dispersión de la especie L. fulica y los Veronicelidos simpátricos, es relevante avanzar 
en el conocimiento de los moluscos que cumplen el rol de hospedadores intermediarios de especies parásitas haciendo énfasis en el estudio de L. fulica y las babosas Veronicellidae que adquieren importancia por su implicancia en la sanidad humana y animal, además de su impacto ambiental por afectar las economías regionales.

\subsection{OBJETIVOS}

\section{Objetivos Generales:}

- Evaluar la distribución y dispersión de Lissachatina fulica (Gasterópoda, Achatinidae) desde su introducción en el área de Puerto Iguazú, Misiones hasta la actualidad.

- Determinar el rol que cumple en la transmisión de helmintos parásitos, con especial énfasis en los nematodes metastrongylidos.

Para alcanzar estos objetivos generales se plantearon los siguientes objetivos específicos:

En relación a los hospedadores:

a) Relevar la presencia de L. fulica en la ciudad de Puerto Iguazú.

b) Complementar el estudio con el relevamiento y análisis de las distintas especies de Veronicellidae simpátricas de L. fulica.

c) Determinar la distribución temporal y espacial de los hospedadores relevados.

d) Analizar y evaluar la distribución de tallas de los especímenes relevados en función del tiempo y de las variables ambientales.

e) Elaborar mapas de distribución de nichos ecológicos de L. fulica.

En relación a los parásitos:

f) Identificar y describir los helmintos parásitos de los gasterópodos de las familias Achatinidae y Veronicellidae en la ciudad de Puerto Iguazú.

g) Calcular los parámetros ecológicos para cada especie parásita.

h) Analizar la relación entre los parámetros poblacionales y las tallas de los hospedadores.

i) Analizar la influencia de los componentes temporal y ambiental en la distribución parasitaria. 


\subsection{Hipótesis y Predicciones derivadas}

En relación a los hospedadores:

Hipótesis 1: La expansión de L. fulica se relaciona directamente con el lapso de tiempo transcurrido.

Predicción 1: Anualmente se identificarán nuevas áreas foco de L. fulica, incrementando así la distribución espacial aceleradamente.

Hipótesis 2. Los factores ambientales (e.g. temperatura y precipitaciones) influyen en el crecimiento corporal de los hospedadores.

Predicción 2. Las cohortes tendrán un mayor crecimiento luego de los períodos de hibernación y estivación.

En relación a los parásitos:

Hipótesis 3: Lissachatina fulica y los Veronicelidos (e.g. Phyllocaulis variegatus) se comportan como hospedadores intermediarios de nematodes Metastrongyloidea.

Predicción 3: Los hospedadores analizados se encontrarán parasitados por nematodes Metastrongyloidea.

Hipótesis 4: La distribución parasitaria es dependiente de la talla de los hospedadores.

Predicción 4: Las tallas mayores de hospedadores mostrarán prevalencias e intensidades medias de infección más altas.

Hipótesis 5: La distribución parasitaria está influenciada por la biología del hospedador y las características del ambiente.

Predicción 5: Las prevalencias e intensidades medias en los hospedadores, serán mayores luego de los períodos de hibernación y estivación. 

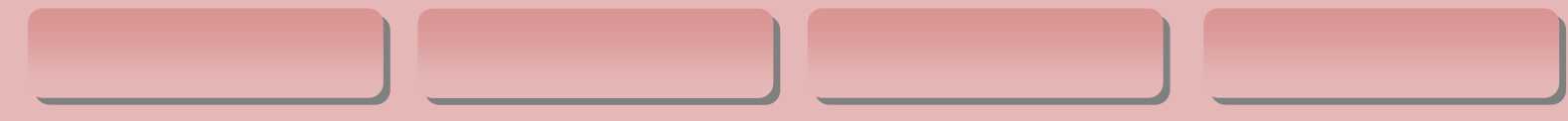

\section{Metodología}

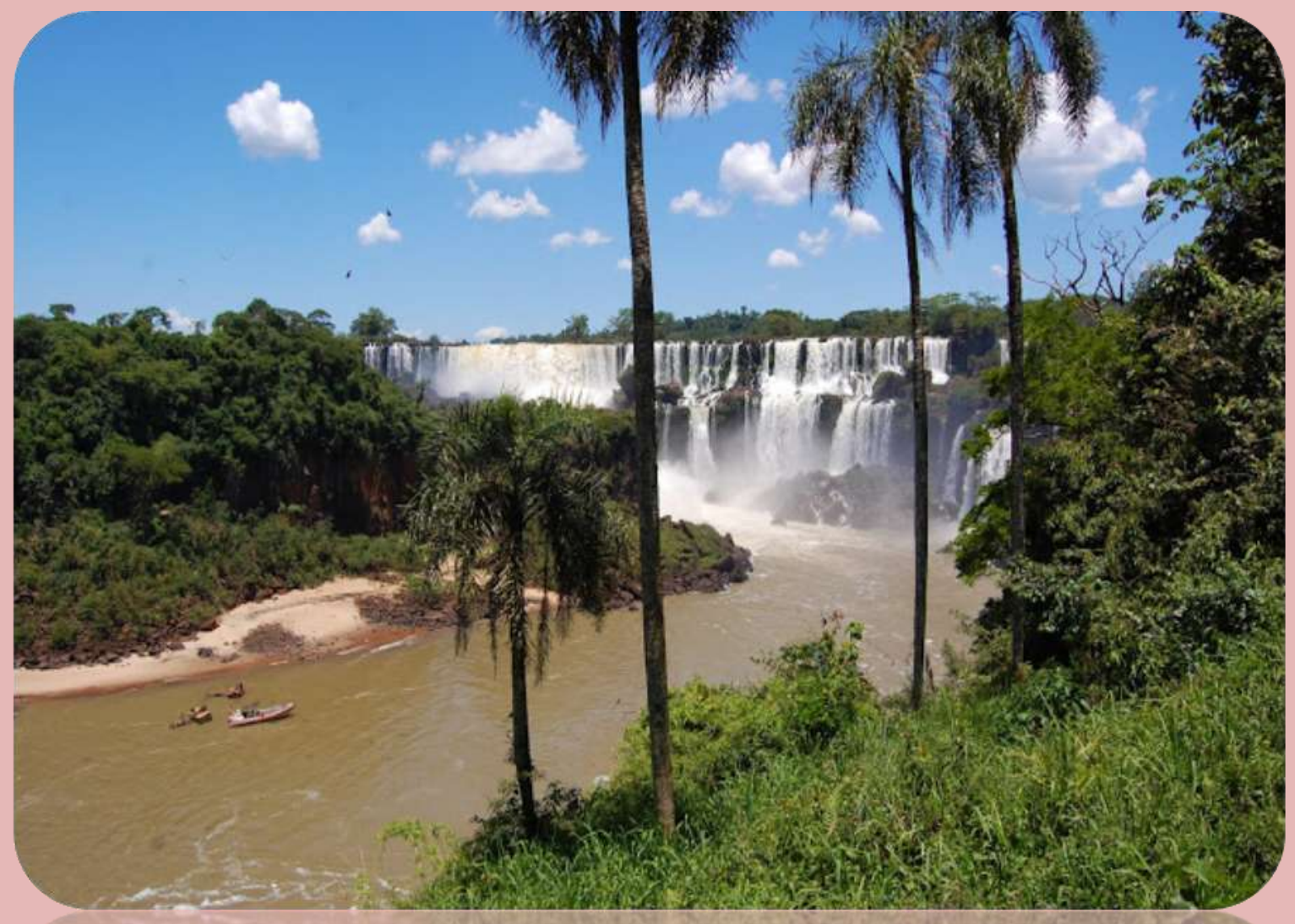




\section{METODOLOGÍA GENERAL}

\section{1. ÁREA DE ESTUDIO}

\section{Caracterización}

El Bosque Atlántico (BA) es un complejo de ecorregiones terrestres que abarcan la costa atlántica de Brasil, el este de Paraguay y el noreste de la Argentina (Provincia de Misiones). Debido a su gran extensión latitudinal y a las diferentes condiciones geomorfológicas, el BA puede dividirse en 15 ecorregiones boscosas, que en su conjunto, están relativamente aisladas de otras áreas boscosas de Sudamérica. De las 15 regiones ecológicas en las que se divide el BA, la región más extensa es el Bosque Atlántico de Alto-Paraná o Selva Paranaense que ocupa alrededor de 471.204 km² (Di Bitetti et al., 2003).

La ecorregión Selva Paranaense se caracteriza por su clima subtropical debido a la lejanía de la costa oceánica. Presenta veranos muy cálidos e inviernos con intenso frío en un corto período de tiempo, y precipitaciones abundantes. La temperatura media anual es de $16-22^{\circ} \mathrm{C}$. En el sur de la ecorregión, las heladas son comunes durante los meses de invierno (junio-agosto), especialmente a elevadas altitudes. Las precipitaciones oscilan entre 1.000-2.200 $\mathrm{mm}$ por año. Estos altos registros pluviométricos son el resultado de los vientos húmedos provenientes del Océano Atlántico, siendo la humedad relativa entre 75 y $100 \%$ con importantes rocíos nocturnos (Di Bitetti et al., 2003).

La Selva Paranaense ha sufrido una reducción de su superficie en los últimos años, ocupa actualmente un 35\% del territorio de Misiones, y las causas más graves de su reducción son la deforestación indiscriminada y la quema para llevar a cabo prácticas agrícolas.

El área de estudio seleccionada en la provincia de Misiones fue la ciudad de Puerto Iguazú ( $\left.25^{\circ} 36^{\prime} \mathrm{S}, 5^{\circ} 35^{\prime} \mathrm{O}\right)$, la cual se encuentra circundada por dos grandes ríos, el Paraná al oeste y el Iguazú al norte (Figura 5. A-B). La ciudad de Puerto Iguazú comprende zonas periurbanas y rurales, y una amplia superficie cubierta por áreas destinadas a la conservación del bosque nativo (parques y reservas). Internamente corren varios cursos de agua entre los que se encuentran los arroyos Tacuara, Mariposa y Panambi, que desembocan en el río Paraná, y los arroyos Cañadón y San Ramón que desembocan en el río Iguazú (Ministerio de Turismo de Misiones). 


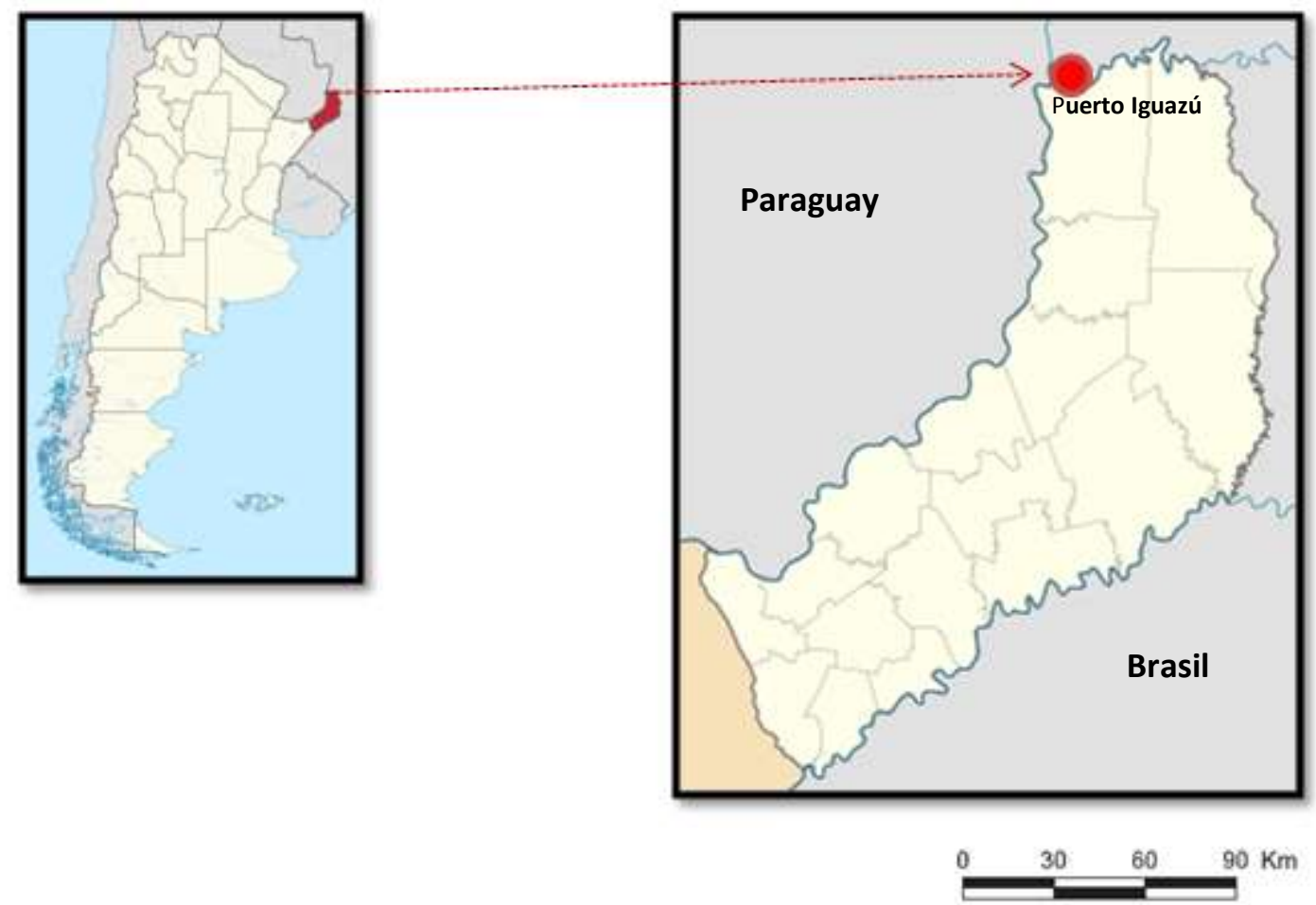

(B)

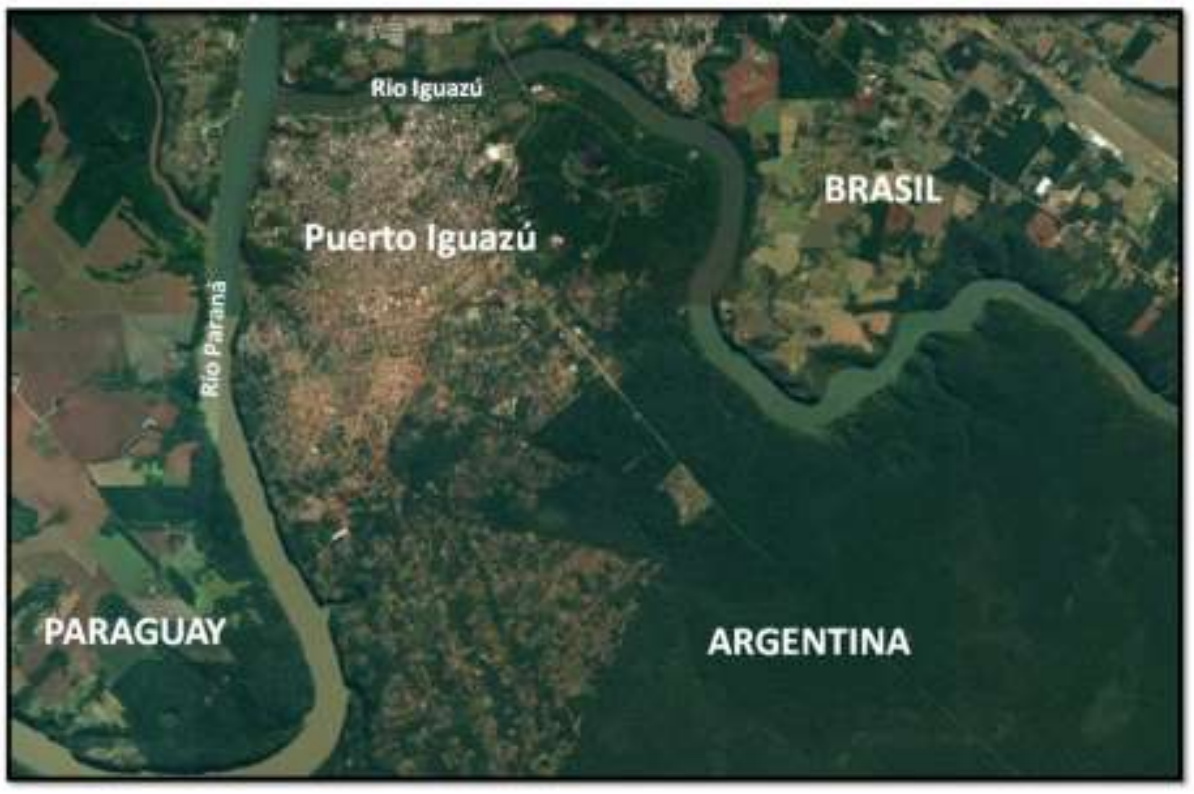

Figura 5. A) Ubicación geográfica de la ciudad de Puerto Iguazú. B) Imagen satelital de Puerto Iguazú. 


\subsection{HOSPEDADORES}

\section{Caracterización de las especies hospedadoras}

\section{Lissachatina fulica}

Tiene una concha de forma cónica puntiaguda con la espira alargada, generalmente hay 7-9 verticilos y rara vez 10. Es de color pardo con marcas o bandas longitudinales oscuras e irregulares, los juveniles son más claros con bandas amarillas. Pueden llegar a medir hasta $208 \mathrm{~mm}$ de alto y $160 \mathrm{~mm}$ de diámetro y en promedio la concha en adultos es de $120 \mathrm{~mm}$ de longitud, con un diámetro de $60 \mathrm{~mm}$ (Correoso Rodríguez, 2006). Es la especie de moluscos terrestre más grande del mundo. La mayor parte del crecimiento promedio es de $5 \mathrm{~mm}$, y posee un crecimiento mensual de $15 \mathrm{~mm}$, que se produce en los primeros seis meses, pero continúa creciendo durante un año (Fischer et al., 2010). Pueden vivir hasta 10 años bajo condiciones controladas, pero usualmente tienen un promedio de vida de 5-7 años (Fischer \& Costa, 2010). Llegan a alcanzar un peso de hasta 600 gramos y hay un polimorfismo evidente. Una de las características más importantes para la identificación de L. fulica es la columella, que se tuerce o termina abruptamente (Skelley et al., 2010).

Las condiciones ambientales, pueden inducir a la hibernación y estivación, lo cual puede promover a cambios fisiológicos en L. fulica y afectar su desarrollo (Albuquerque et al., 2007).

Es una especie hermafrodita protándrica, después de la cópula los especímenes son capaces de almacenar esperma, posibilitando sucesivas puestas de huevos después de un solo apareamiento, hasta seis puestas por año. Pueden poner hasta varios cientos de huevos por puesta (30-1.000) a $25 \mathrm{~cm}$ de profundidad de la tierra, su color es blancoamarillo, con forma ovalada. La incubación depende de la temperatura, las crías nacen entre los 5 a 21 días luego de la puesta y una vez que eclosionan se disponen en forma agregada alrededor de un recurso alimenticio (Fischer \& Costa, 2010).

Este molusco es de hábitos nocturnos, aunque en épocas de humedad se lo puede ver de día. Vive en refugios, a los cuales vuelve cuando termina de alimentarse. Ante condiciones desfavorables produce una membrana mucosa y transparente, el epifragma, que cubre la abertura y lo aísla del medio, entrando en periodo de hibernación o estivación, hasta que las condiciones vuelvan a ser favorables (Gutiérrez Gregoric et al., 2011). 
Lissachatina fulica consume básicamente vegetación pero puede llegar a desarrollar la coprofagia. Aunque es una especie de zonas cálidas y semiáridas puede adaptarse a cualquier tipo de hábitat, desde zonas intervenidas por el hombre para desarrollar la agricultura y la ganadería, hasta pantanos y zonas urbanas donde exista vegetación. Esta especie prefiere sitios no expuestos directamente a la luz solar, con alta humedad ambiental (70\%) y temperatura entre 18 y $20^{\circ} \mathrm{C}$ (Matinella et al., 2009). Se distribuye mayormente en plantaciones de cultivos, jardines, parques, hojarascas en descomposición y orillas de cursos de agua con vegetación (Correoso Rodríguez, 2006) (Figura 6).
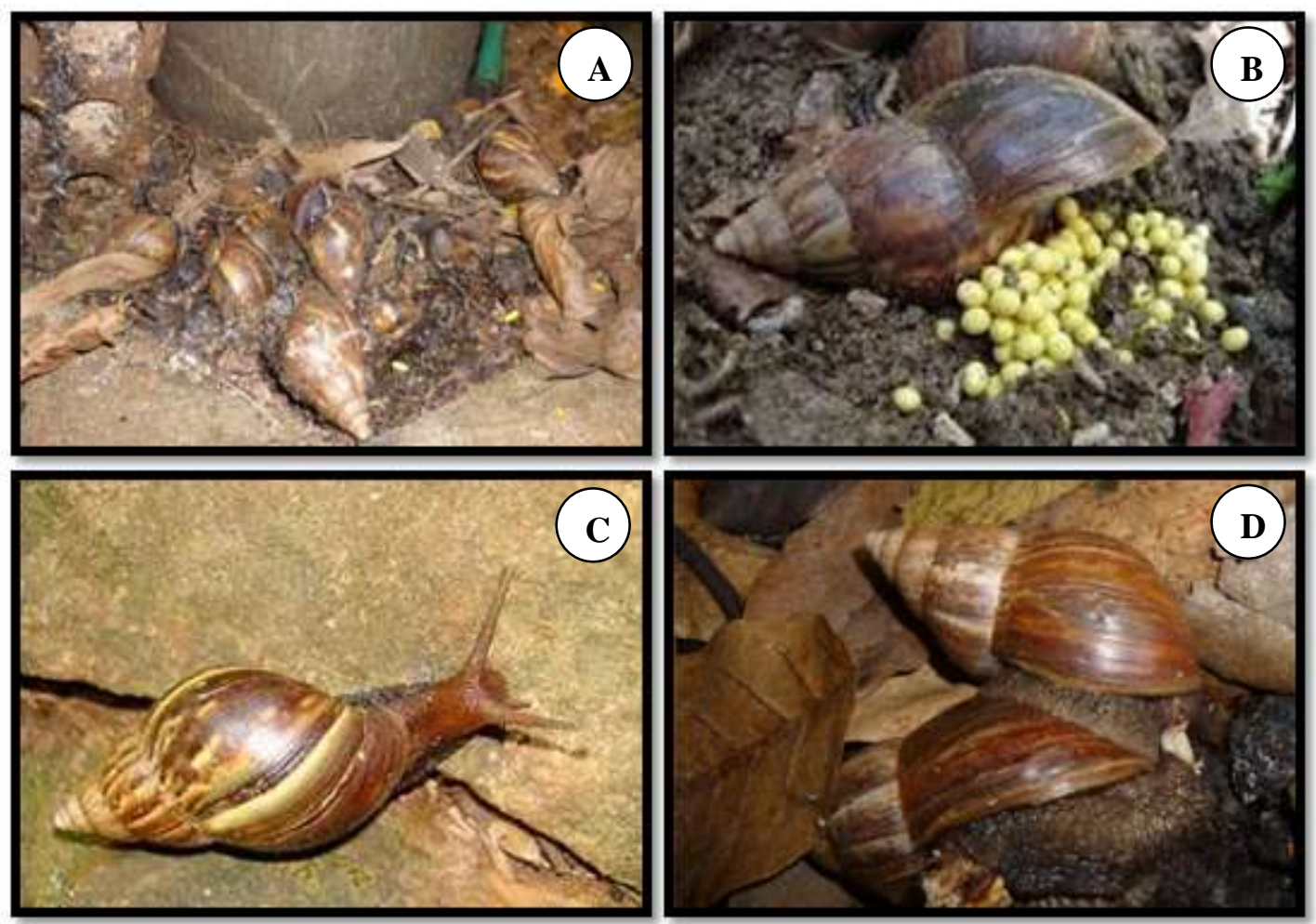

Figura 6. Lissachatina fulica: A). Grupo de ejemplares al pie de un árbol. B) Puesta de huevos. C) Ejemplar de un adulto juvenil. D) Ejemplares en cópula.

\section{Babosas Veronicellidae}

Son gasterópodos terrestres desprovistos totalmente de concha y poseen el cuerpo achatado dorso-ventralmente. La respiración se produce a través del tegumento. Poseen dos pares de tentáculos, los inferiores son cortos y bifurcados y los tentáculos superiores son largos contráctiles y en su parte superior se encuentran los ojos (Rodrigues Gomes, 2007; Ohlweiler et al., 2010). 
Son hermafroditas con fecundación cruzada simultánea, alternada o autofecundación. Su ciclo de vida, la velocidad de reproducción y su crecimiento corporal son condicionados por las condiciones climáticas, la luz y la comida disponible.

Su hábito es permanecer en lugares sombríos y húmedos junto a las viviendas. Pueden encontrarse enterrados en troncos de árboles y hojarascas en avanzado estado de descomposición. Tienen hábitos nocturnos o crepusculares evitando así las altas temperaturas del día (Rodrigues Gomes, 2007; Ohlweiler et al., 2010). Sin humedad, la babosa no puede producir moco y por lo tanto no puede desplazarse. En épocas secas se refugia en el suelo para esperar la vuelta de las lluvias. Algunas especies producen una membrana de aspecto parecido a una cáscara debajo de su tegumento superior para prevenirlas de desecarse por completo (Rodrigues Gomes, 2007).

La clasificación a nivel de especie se basa principalmente en la morfología del sistema reproductor masculino: aspecto general del pene y glándulas penianas accesorias. Las aberturas genitales masculinas y femeninas están separadas, el gonoporo masculino se encuentra cerca de la base de los tentáculos inferiores, y el femenino en la región ventrolateral del cuerpo (Santin \& Miquel, 2015). La oviposición se realiza en sustratos húmedos, y los huevos translúcidos forman cordones gelatinosos.

\section{Phyllocaulis variegatus}

El patrón de pigmentación del tegumento va desde una coloración marrón clara a oscura. Pueden llegar a superar los $10 \mathrm{~cm}$ de longitud total. Presenta un pie bien desarrollado a comparación de otras babosas de otras familias. El pene es ancho, deprimido, con un borde dentado, rodeado de un elemento accesorio que es la "espata" de aspecto liso, con bordes espinados y extremo algo acuminado. Presenta glándulas penianas con túbulos diferenciados (Santin \& Miquel, 2015).

Actualmente se encuentra distribuida en Buenos Aires, Entre Ríos, Misiones y Ciudad Autónoma de Buenos Aires (Santin \& Miquel, 2015) (Figura 7. A-B).

\section{Latipes erinaceus}

Esta especie generalmente presenta un tegumento de color marrón amarillento con manchas negras dispersas irregularmente. Su tamaño corporal no supera $\operatorname{los} 7 \mathrm{~cm}$ de largo. A diferencia de $P$. variegatus presenta un pie estrecho. 
El sistema reproductor presenta un pene cordiforme de base ancha, con su extremo dirigido hacia abajo donde se disponen una serie de tubérculos pequeños, la estructura accesoria "espata" está ausente. Las glándulas penianas presentan túbulos cortos (Santin \& Miquel, 2015).

Se encuentra distribuida en Tucumán, Corrientes y Misiones (Santin \& Miquel, 2015) (Figura 7. C-E).
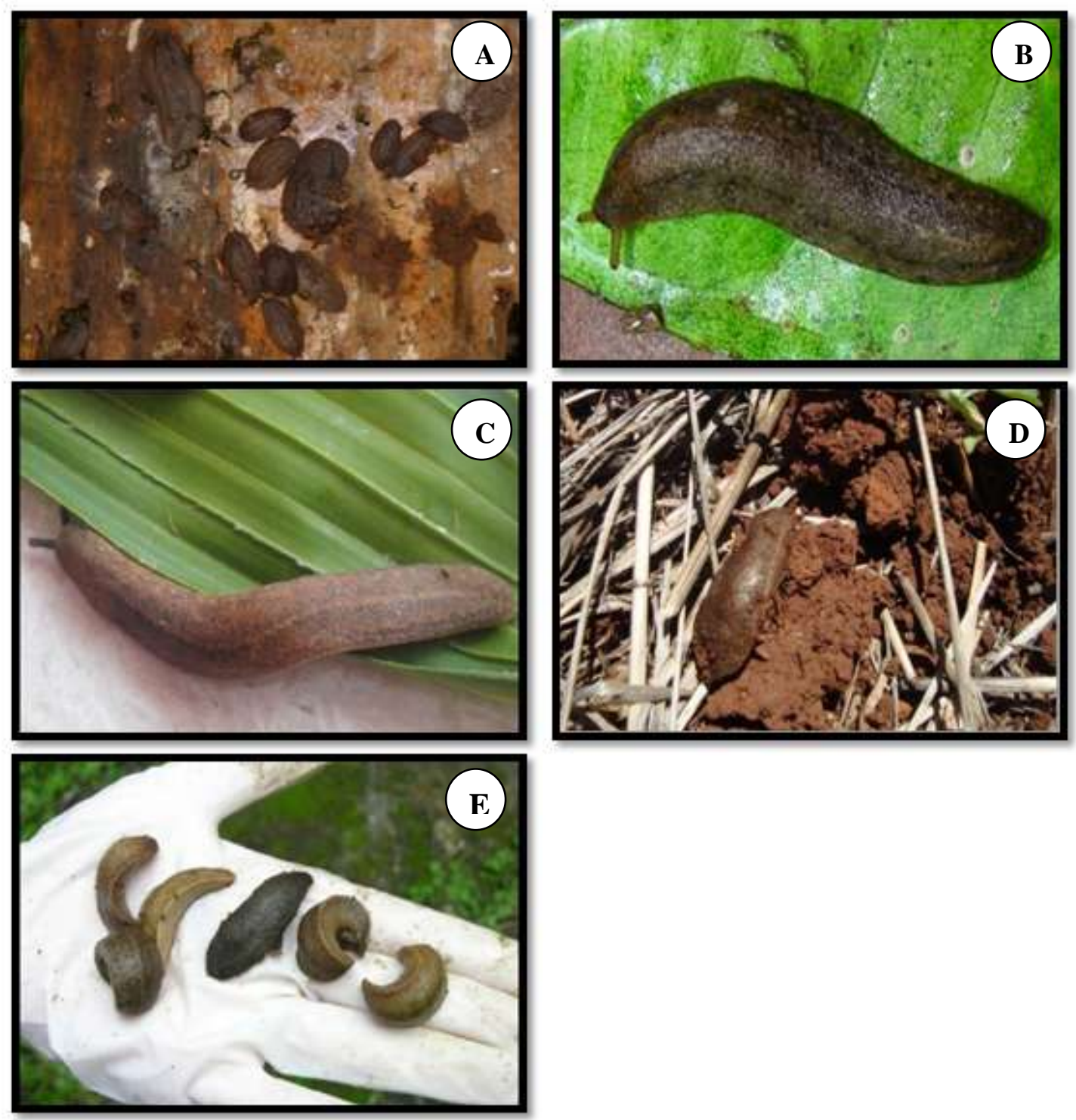

Figura 7. A) Phyllocaulis variegatus agrupados. B) Ejemplar de Phyllocaulis variegatus. C) Adulto de Latipes erinaceus. D) Latipes erinaceus sobre la tierra misionera. E) Ejemplares de Latipes erinaceus. 


\subsection{Trabajo de campo}

\subsection{Diseño de muestreo}

Se realizaron muestreos exploratorios desde mayo a septiembre de 2013, en distintas áreas de la ciudad de Puerto Iguazú, con el fin de detectar la presencia de $L$. fulica y de babosas Veronicellidae, y poder seleccionar las áreas de muestreo.

La ciudad de Puerto Iguazú fue dividida en una grilla de 200 x 200 m (cada uno de los cuadrados que conforman la grilla está formado por cuatro manzanas, y cada punto azul es el sitio donde se muestreo), contabilizando un total de 1.442 celdas (Figura 8. A-B). En la zona urbana se contaron 200 celdas, de las cuales se seleccionaron al azar 12 celdas (la celda 13 y 14 fueron incorporadas el último año, debido a la aparición del caracol gigante africano en esos barrios) cercanas a cursos de agua, utilizando la herramienta Hawths tools de Arcgis 10.1 (Figura 9).

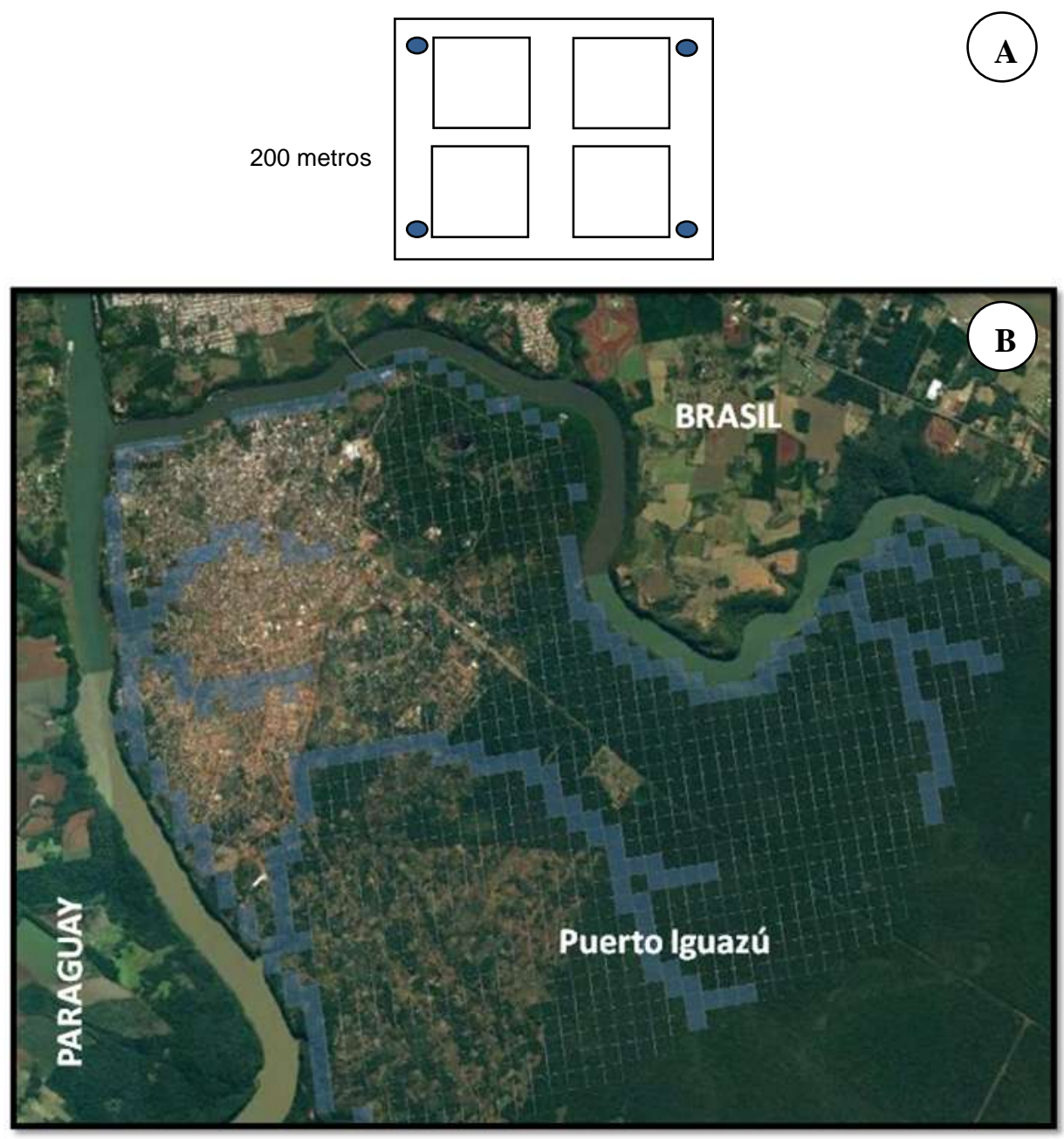

Figura 8. A) Caracterización de la grilla de 200 por 200. B) Mapa de la ciudad de Puerto Iguazú con las grillas. 
El área control está conformada por zonas de quintas y granjas, donde se contabilizaron un total de 120 celdas. Para evaluar la dispersión de las especies se seleccionaron 5 celdas de 200 x 200 en una zona no urbana distante $2 \mathrm{~km}$ aproximadamente de los sitios urbanos. Se trazó una transecta entre el punto más cercano y más distante a la zona urbana (Figura 10).

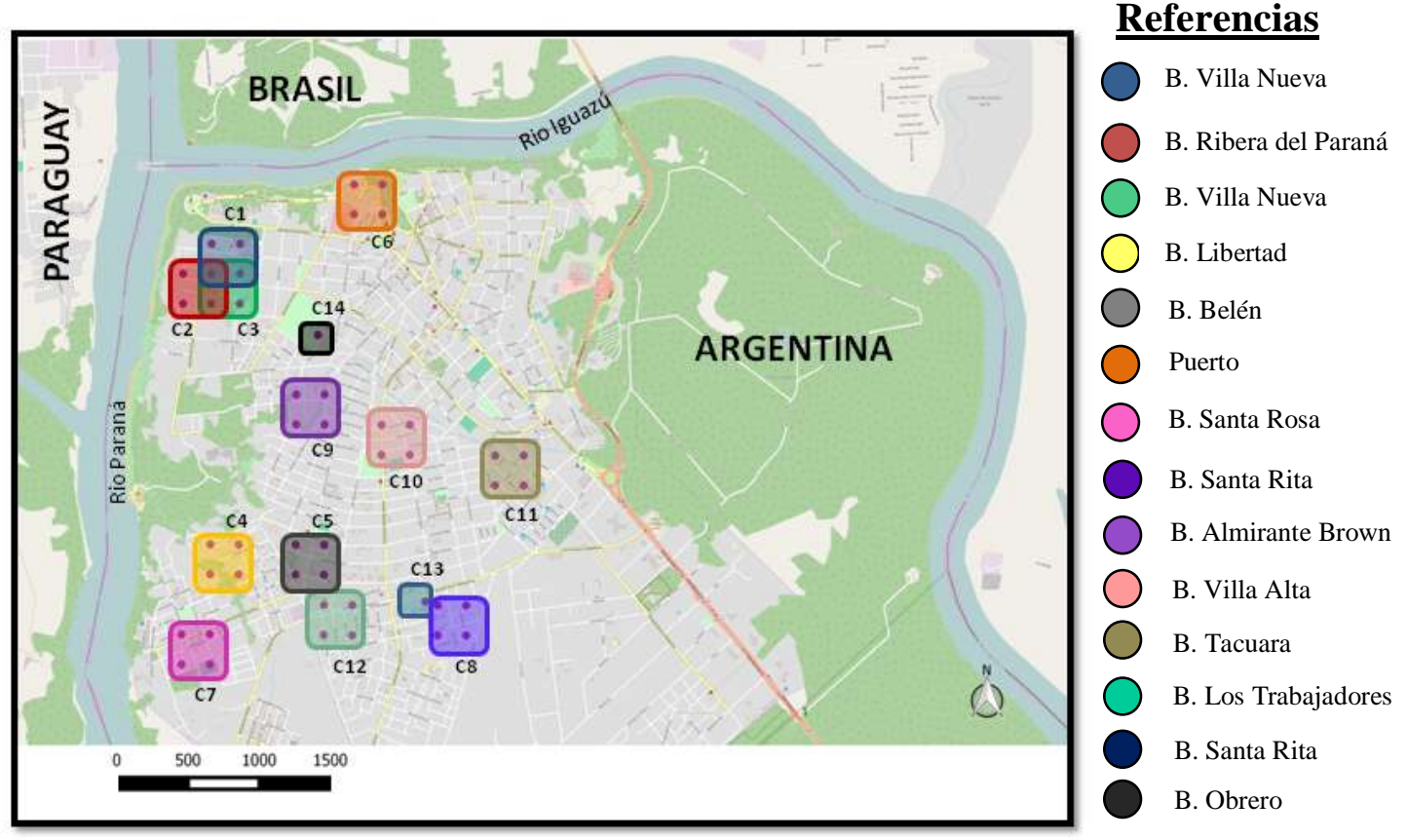

Figura 9. Mapa de la ciudad de Puerto Iguazú mostrando las celdas seleccionadas.

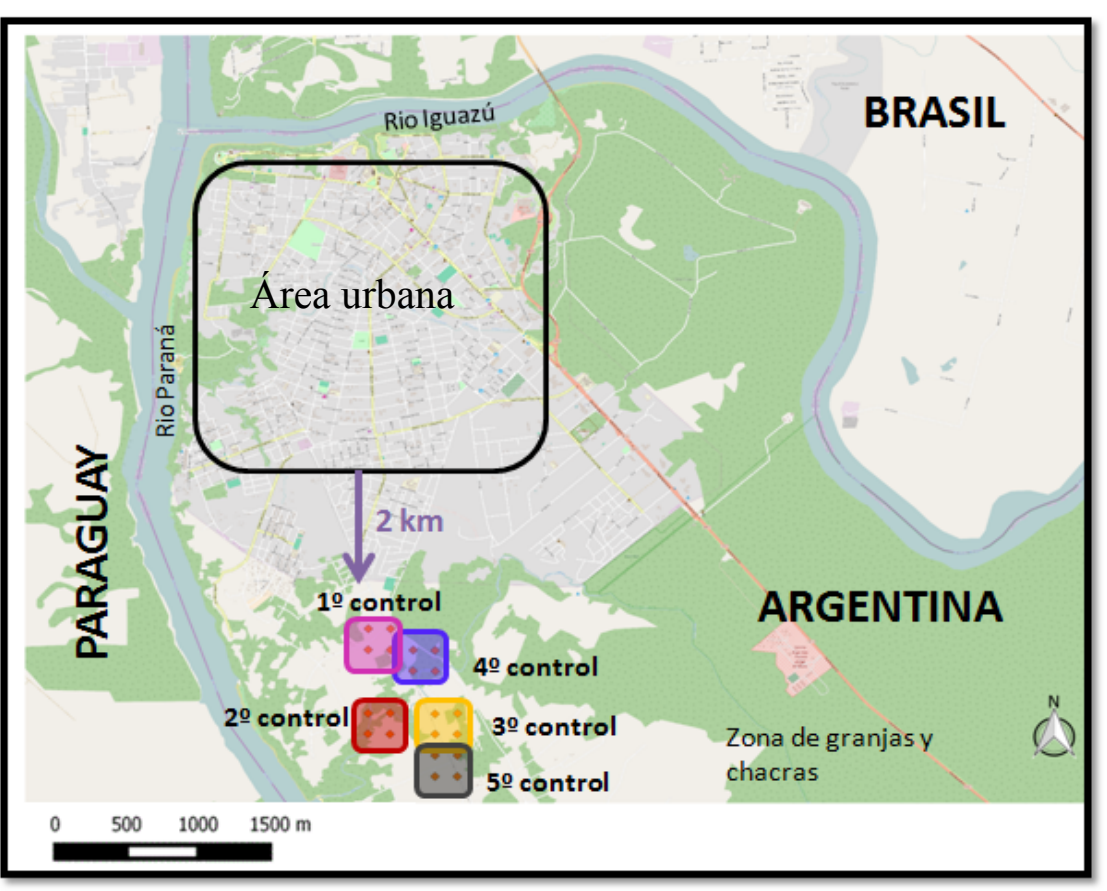

Figura 10. Mapa de la ciudad de Puerto Iguazú mostrando las celdas de control. 


\section{Sitios de muestreos en el área urbana}

\section{Celda 1}

Se encuentra situada en el barrio Villa Nueva, cercano al arroyo Mariposa que desemboca en el río Paraná. Este barrio fue unos de los primeros focos de aparición de L. fulica en el 2010. Las viviendas son en su mayoría de material, rodeadas de vegetación abundante típica de selva y bosque en galería (Figura 11. A).

\section{Celda 2}

Este sitio se encuentra localizado en el barrio Ribera del Paraná delimitado por uno de los márgenes laterales del río Paraná. Fue citado foco de aparición de L. fulica en el año 2010. La vegetación de los alrededores es más densa, con lugares impenetrables. La orilla del río Paraná, se encuentra a unos 200 metros de esta área en la cual la pesca es habitual entre los habitantes de la ciudad. Se observó un área descuidada por la falta de recolección de residuos que se depositan en terrenos baldíos (Figura 11. B).

\section{$\underline{\text { Celda } 3}$}

Área ubicada en el barrio Villa Nueva, lugar donde se encuentra la escuela primaria $\mathrm{N}^{\circ} 164$, que se vio afectada por la presencia de gran cantidad de L. fulica. La problemática observada causó preocupación por parte de la comunidad educativa, lo que motivó la realización de campañas de concientización para la detección del caracol gigante africano y sobre medidas de prevención necesarias por riesgo sanitario (Figura 11. C).

\section{$\underline{\text { Celda } 4}$}

Este sitio se encuentra en las cercanías del arroyo Panambí, que desemboca en el río Paraná. Es el barrio Libertad que corresponde a zonas de chacras. Sólo se realizó un muestreo dado el difícil acceso por el deterioro de los caminos.

\section{Celda 5}

Esta celda está localizada en el barrio Belén, muy próximo al Instituto Nacional de Medicina Tropical. Las casas en su mayoría son de material rodeadas de abundante vegetación. Este área corresponde a la zona interna de la ciudad de Puerto Iguazú. 


\section{Celda 6}

Esta zona corresponde al área portuaria de la ciudad. Cercano a la misma se encuentra el edificio de la Administración de Parques Nacionales, Aduana, y Prefectura Naval Argentina.

\section{Celda 7}

Corresponde al barrio Santa Rosa, que se encuentra en los márgenes del río Paraná. Las casas de madera están situadas sobre áreas con pendientes, por lo cual las intensas lluvias periódicas hacen de ésta un área inundable. La población del lugar pesca habitualmente.

\section{Celda 8}

Pertenece al barrio Santa Rita. Este área tiene casas de material con escasa vegetación circundantes.

\section{Celda 9,10 y 11}

Estas celdas se encuentran sobre el arroyo Tacuara, sin embargo ese barrio fue modificado cuando comenzó el entubamiento del arroyo, y las casas fueron removidas del lugar para ser reubicadas en otro sitio de Puerto Iguazú. (Figura 11. D)

\section{Celda 12}

Se encuentra en el Barrio Los Trabajadores. Esta área tiene casas de material con escasa vegetación circundantes.

\section{$\underline{\text { Celda } 13}$}

Esta celda incluye puntos de muestreo en el barrio Santa Rita que fueron incluidos como nuevos puntos de aparición de L. fulica en el año 2015. Las casas son de material y escasa vegetación circundante.

\section{Celda 14}

Los puntos de muestreo se encuentran en el barrio Obrero, y fueron incluidos como nuevos puntos de aparición de L. fulica en el año 2015 (Figura 11. E-F). 

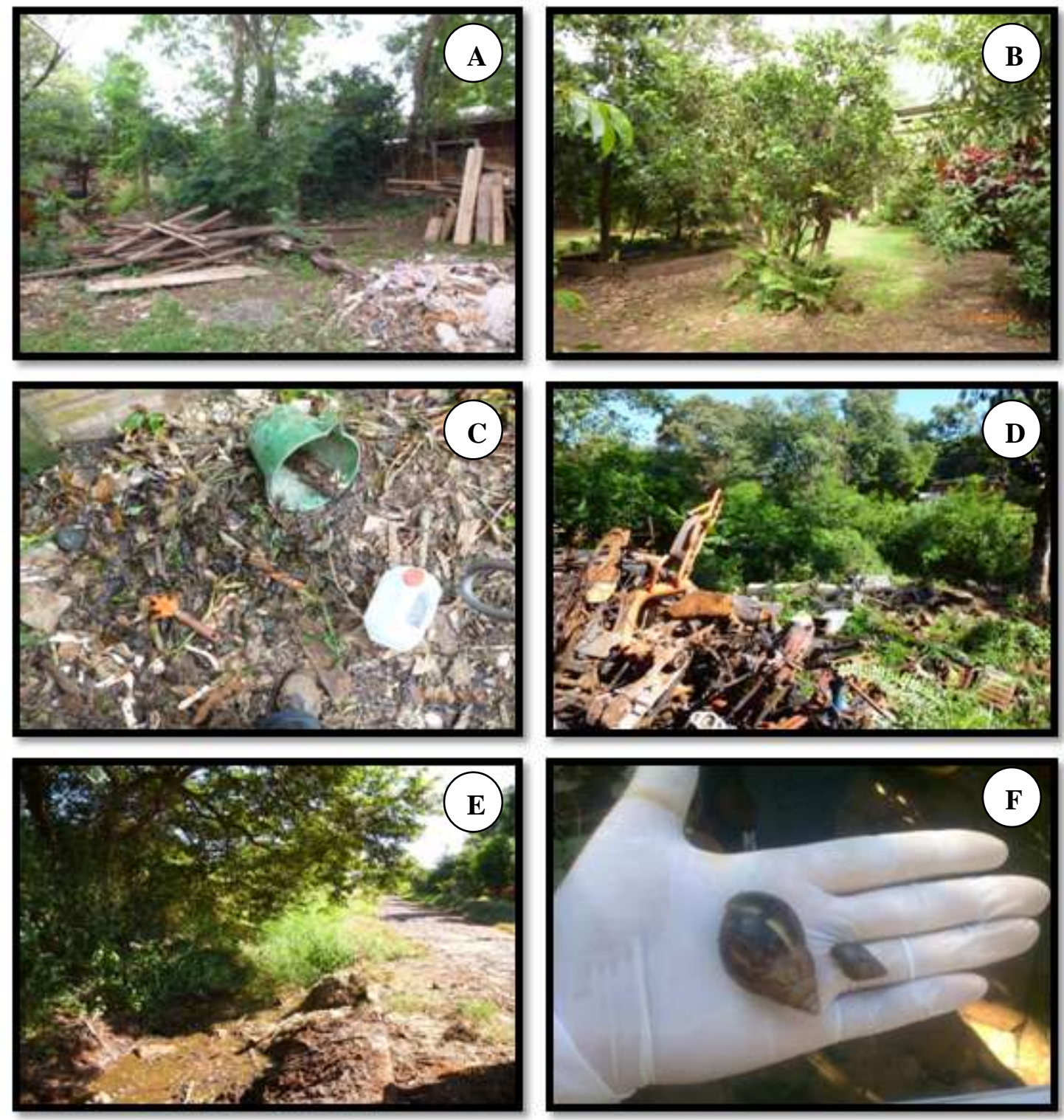

Figura 11. A) Sitio de muestreo correspondiente a celda 1. B) Sitio de muestreo correspondiente a celda 2. C) Sitio de muestreo correspondiente a celda 3. D) Sitio de muestreo correspondiente a las celdas 9,10 y 11. E) Sitio de muestreo que corresponde a celda 14. F) Ejemplar de Lissachatina fulica recolectado en celda 14.

\section{Sitios de muestreos en el área control}

El área control se localiza en la zona de granja y quinta de la ciudad, donde hay plantaciones. Este área se encuentra alejada de la zona urbana, ya que sus caminos son de tierras, y se hallan muy deteriorados, lo que imposibilitan el paso hacia esta zona. Las lluvias frecuentes en ciertos periodos del año empeoran este escenario, por lo cual los muestreos se pudieron realizar una vez al año en el bimestre de febrero-marzo (Figura 12. A-F). 

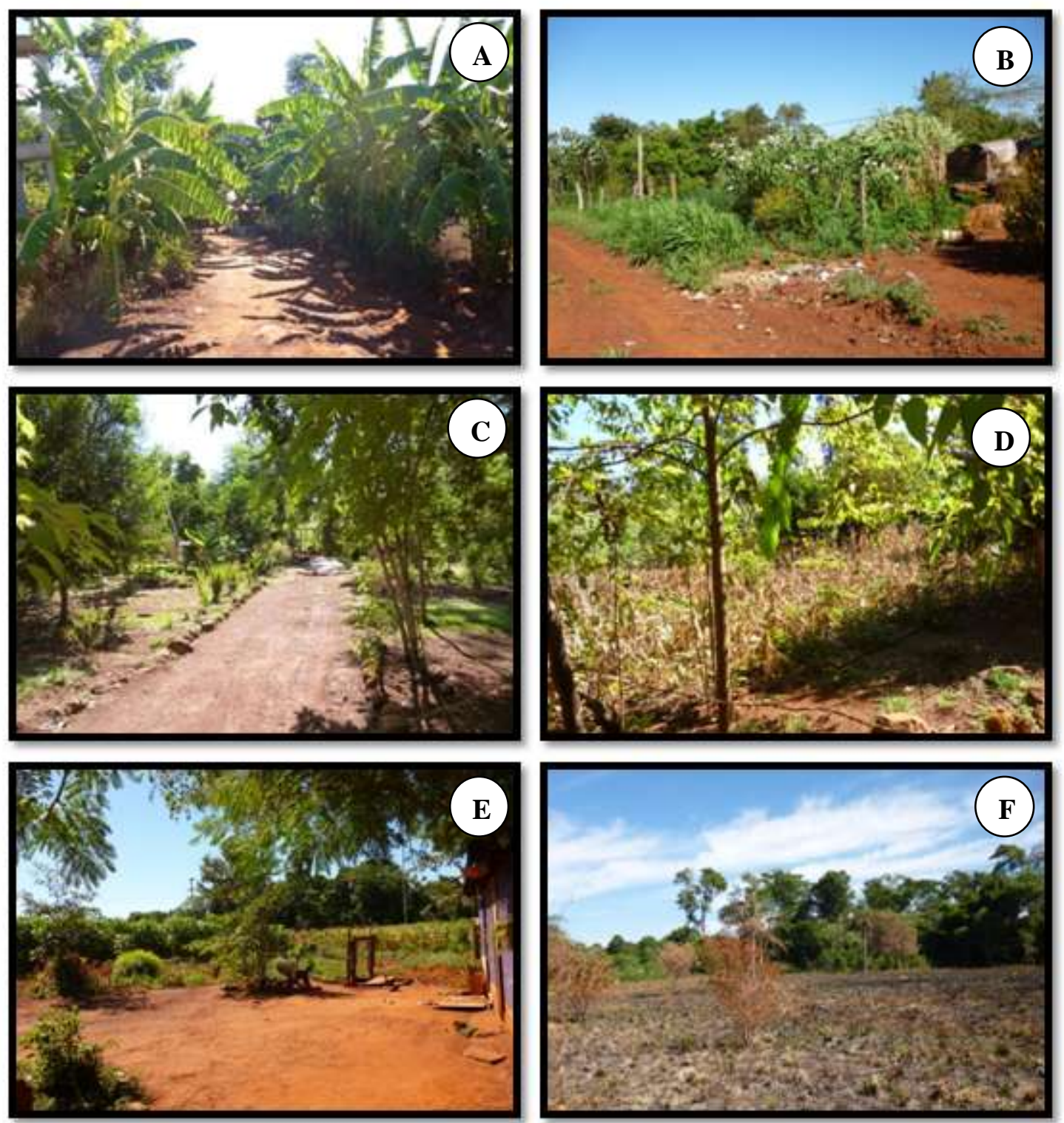

Figura 12. A-B) Primer área control. C) Segunda área control. D) Tercer área control. F) Cuarta área control. F) Quinta área control.

\subsubsection{Variables ambientales relevadas}

Las variables abióticas consideradas fueron las medias bimestrales de la temperatura (mínima y máxima), precipitaciones y humedad relativa (máxima y mínima) de cada año de muestreo, la cuales fueron calculadas a partir de las variables ambientales proporcionadas por el Servicio Meteorológico Nacional, Aéreo Iguazú (Dr. Oscar Salomon - Instituto Nacional de Medicina Tropical - Expte $N^{\circ} 150.139$ ) (Anexo IV). 


\subsubsection{Recolección de los hospedadores}

Los hospedadores fueron colectados por dos personas siguiendo el método de captura por remoción (Rabinovich, 1980) mediante colecta manual, ayudados por elementos de jardinería tales como rastrillos y palas, en la época de altas y bajas temperaturas (período de estivación: febrero-marzo; período de hibernación: juniojulio) ya que los mismos se encuentran enterrados hasta $15 \mathrm{~cm}$ de la superficie del suelo. Los muestreos se realizaron durante las primeras horas de la mañana (8 a 10 am), dada la imposibilidad de acceder a las viviendas en horario nocturno.

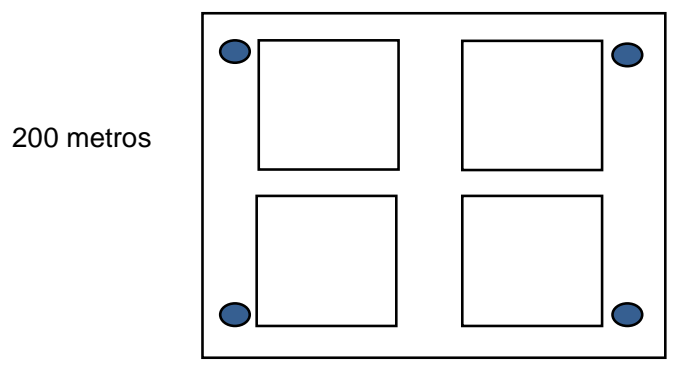

Figura 13. Caracterización de la grilla de 200 por 200.

Los extremos o vértices (Figura 13) de cada una de las 12 celdas fueron recorridos en contra de las manecillas del reloj. En cada punto fueron tomadas las coordenadas con un GPS. El esfuerzo máximo de muestreo en cada punto fue de 15 minutos. Se realizaron muestreos cada dos meses, desde octubre del 2013 a noviembre de 2015 (octubre-noviembre, febrero-marzo y junio-julio), que fueron denominados bimestres, con bimestres intermedios de reposo (diciembre-enero, abril-mayo, agostoseptiembre) en donde no se realizaron relevamientos, estos "períodos de reposo" fueron arbitrariamente seleccionados para permitir la recuperación poblacional.

Los ejemplares colectados en cada una de las celdas fueron colocados en recipientes de plástico etiquetados, y llevados al laboratorio para ser procesados. Fueron colectados un total de 995 L. fulica y 134 babosas de la familia Veronicellidae.

\subsection{LABORATORIO}

\subsubsection{Estudio de los hospedadores}

En el laboratorio, después de $48 \mathrm{hs}$ los hospedadores de cada celda fueron separados en 2 grupos para ser procesados utilizando dos técnicas diferentes: 
1) evisceración y 2) digestión artificial. Los caracoles fueron medidos (desde el ápice hasta el extremo posterior de la concha) con un calibre de $0,1 \mathrm{~mm}$ de precisión. Las babosas fueron medidas con una regla de $0,1 \mathrm{~mm}$ de precisión. Se establecieron cuatro rangos de talla de $3 \mathrm{~cm}$ : 1) [0-3) $\mathrm{cm} \mathrm{2)} \mathrm{[3-6)} \mathrm{cm} \mathrm{3)} \mathrm{[6-9)} \mathrm{cm} \mathrm{4)} \mathrm{[9-12)} \mathrm{cm} \mathrm{(Figura} 14$. A).

\section{1) Evisceración}

Los caracoles y babosas fueron colocados en tarros etiquetados con fechas, que contenían cristales de mentol; una vez colocados allí se llenó todo el recipiente con agua corriente. Los tarros fueron llevados a heladera durante $48 \mathrm{hs}$ para acelerar el proceso de relajación. Pasado ese tiempo los caracoles fueron fijados durante $24 \mathrm{hs}$ en formaldehido al $10 \%$, y posteriormente conservados en alcohol al $70 \%$ para luego ser examinados. Las babosas fueron conservadas solo en alcohol al 70\%. Para extraer las vísceras de los caracoles, se procedió a romper la concha con un martillo de madera. Se separó la cavidad del manto, estómago, hepatopáncreas, intestino y los órganos reproductivos, todos ellos fueron colocados en cápsulas de Petri y luego se observaron bajo microscopio estereoscópico Trino Arcano ZTX (6.3-40 X). Los helmintos hallados en cada órgano se contabilizaron y luego se conservaron en alcohol 70\% para identificaciones posteriores.

Las babosas fueron colocadas en posición lateral en cápsulas de Petri, se realizó un corte longitudinal en el margen lateral donde se encuentra el gonoporo femenino, para extraer los órganos. La cavidad del cuerpo fue examinada bajo microscopio estereoscópico Trino Arcano ZTX en búsqueda de parásitos (Pritchard \& Kruse, 1982).

\section{2) Digestión artificial}

Los moluscos fueron procesados sin previa fijación. Para remover las vísceras, pie y cabeza de los caracoles, se procedió a romper la concha con un martillo de madera (Figura 14. B). Las babosas fueron colocadas en su totalidad en la solución de digestión.

Se prepararon $1000 \mathrm{ml}$ de solución de digestión utilizando $993 \mathrm{ml}$ de agua destilada y $7 \mathrm{ml}$ de ácido clorhídrico puro, posteriormente $5 \mathrm{mg}$ de pepsina fueron disueltos en la solución (Wallace \& Rosen, 1969). Para que la mezcla sea homogénea se procedió a colocarla en un agitador magnético a $37^{\circ} \mathrm{C}$ durante 30 minutos (Figura 14. C). Cada caracol y babosa viva fue colocada en vasos de precipitado que contenían 15 ml de la solución de digestión. Para acelerar el proceso de digestión, fueron llevados a 
un agitador magnético con una temperatura constante de $37^{\circ} \mathrm{C}$, durante 4 o 5 horas dependiendo del tamaño del individuo. Para recuperar las larvas, la solución digerida fue sometida al método de Baermann modificado (Figura 14. D). El objetivo de esta técnica consiste en filtrar el contenido digerido: la solución digerida se pasa por un embudo (sostenido en un porta embudo enganchado a un soporte universal) que contiene un colador con poros de $1 \mathrm{~mm}$, y sobre ella se encuentra una gasa que es la que retendrá las vísceras no digeridas. Ese embudo está conectado a una manguera de caucho de $15 \mathrm{~cm}$ de longitud que en el final posee una pinza de Mohr.

El contenido se volcó en el embudo hasta llegar a su tope y se dejó decantar 24 hs. Al día siguiente se tomaron $10 \mathrm{ml}$ de la solución estacionada, y se los centrifugo tres veces a $1.500 \mathrm{rpm}$, en cada paso se descartó el sobrenadante y completándolo con agua destilada hasta los $10 \mathrm{ml}$ del tubo. Luego de estos tres lavados se procedió a observar el contenido en un microscopio óptico Olympus BX51 (40-1000 X).

\subsubsection{Estudio de los helmintos}

Los nematodes fueron aclarados con alcohol glicerinado. Los digeneos fueron teñidos con diferentes técnicas de coloración: carmín clorhídrico o carmín acético, con la adecuada deshidratación en una serie gradual de alcoholes (70, 80, 90, 96 y 100), diafanizados en metil salicilato, y montados en bálsamo de Canadá natural. Las observaciones se realizaron en un microscopio Olympus BX51 equipado con DIC, cámara fotográfica, ocular micrométrico y tubo de dibujo (Figura 14. E). Posteriormente fueron identificados utilizando bibliografía específica (Ash, 1970; Yamaguti, 1975; Gibson et al., 2001; Anderson et al., 2010). Para los estudios taxonómicos, las medidas se aportan en micrómetros.

Los helmintos fueron depositados en la Colección Helmintológica del Museo de La Plata (CHMLP). 

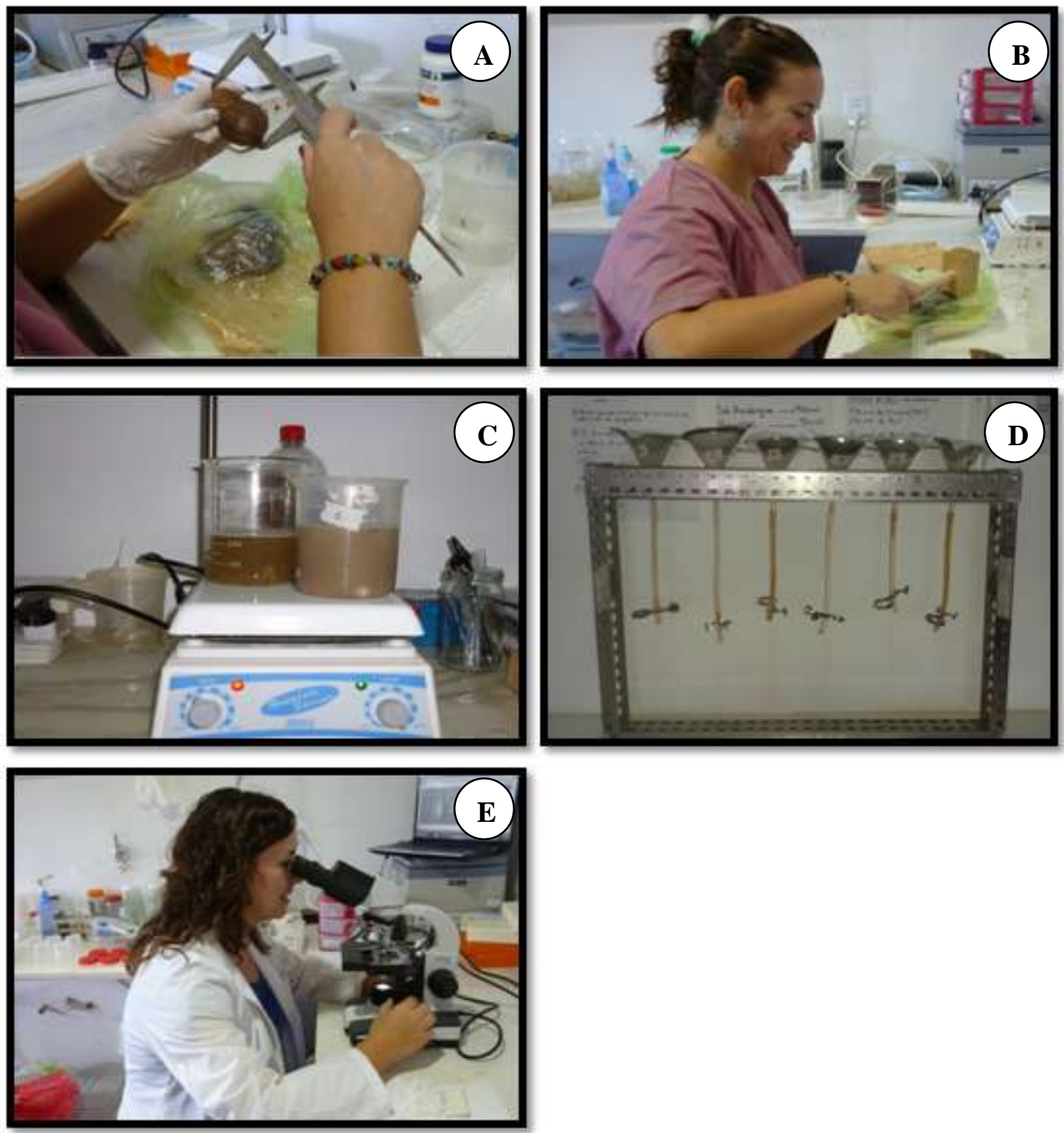

Figura 14. A) Medición de ejemplares de Lissachatina fulica. B) Rompiendo concha de Lissachatina fulica. C) Digestión artificial en agitador magnético. D) Método de Baermann modificado. E) Observación al microscopio óptico (MO).

\subsection{ANÁLISIS DE LOS DATOS}

- Para determinar la distribución temporal y espacial de los hospedadores se contemplaron cada una de las coordenadas que fueron tomadas por celda, en cada bimestre de cada año. Estos datos fueron respaldados por mapas y gráficos.

- La distribución de tallas de los hospedadores en función del tiempo y de las variables ambientales, fueron examinadas por análisis de correspondencia. 
- Se calcularon los parámetros ecológicos prevalencia, abundancia media e intensidad media siguiendo las definiciones de Bush et al. (1997):

Prevalencia (P): número de hospedadores infectados/número de hospedadores analizados.

Abundancia: número de individuos parásitos en un individuo hospedador (puede ser cero).

Abundancia Media (AM): número de individuos parásitos/número de hospedadores analizados.

Intensidad: número de individuos parásitos en un hospedador infectado.

Intensidad Media (IM): número de individuos parásitos/número de hospedadores parasitados.

- Para analizar las relaciones entre: i) la $\mathrm{P}$ total de infección $v s$. el tamaño del hospedador (largo total de la concha), ii) IM vs. el tamaño del hospedador; se utilizó el test no paramétrico de correlación por ranking de Spearman ( $r \mathrm{~s})$. Se midió la prevalencia total de infección considerando intervalos de clases de tamaño del hospedador con un rango $3 \mathrm{~cm}$.

- Para analizar la influencia de las variables ambientales sobre la carga parasitaria se calcularon: i) la $\mathrm{P}$ total de infección vs. cada bimestre, ii) IM vs. cada bimestre; se utilizó el test no paramétrico de correlación por ranking de Spearman ( $r \mathrm{~s}$ ).

- Los mapas de distribución de nicho ecológico se realizó sólo para el caracol africano L. fulica, ya que es la especie hospedadora invasora. Se analizó la distribución geográfica de este hospedador relacionando su presencia-ausencia en Sudamérica, con las características del ambiente (e.g. suelo, clima, condiciones hidrográficas, pendientes del relieve, características de la vegetación).

Ello permitió realizar un análisis geoespacial utilizando el software libre QuantumGIS. Los datos de distribución geográfica (las presencias se obtuvieron de diferentes fuentes bibliográficas y páginas web oficiales) (Anexo V) se observarán en el mapa con un punto negro. Las presencias, más las variables ambientales (Worldclim: Global Climate Date) permitieron elaborar mapas de nichos ecológicos de esta especie hospedadora. Este análisis se realizó utilizando el algoritmo MaxEnt (MaxEnt v.3.3.3; Phillips et al., 2006). El modelado de la distribución potencial de las especies es entonces el proceso de construir una representación espacial de los requerimientos 
ecológicos fundamentales para una especie y extrapolarlos dentro de una región geográfica, constituyendo así el nicho ecológico fundamental de la especie (Scheldeman \& Van Zonneveld, 2011).

Las variables ambientales utilizadas fueron las presentes (1970-2000), y futuras bajo condiciones de cambio climático (2041 a 2060, con un promedio de años de 2050). Los análisis se realizaron sobre cuatro escenarios climáticos denominados RCP (se obtiene del valor de forzamiento relativo $\mathrm{W} / \mathrm{m} 2$, que corresponde con la energía solar media absorbida por metro cuadrado sobre la tierra=emisión de dióxido de carbono), que representan un mayor efecto invernadero en las emisiones de gas en todo el siglo XXI que conducen a altas concentraciones de gases de efecto invernadero a través del tiempo. Estos cuatro escenarios son: RCP 2.6, RCP 4.5, RCP 6.0, RCP 8.5. El RCP 2.6 es un escenario optimista con poca emisión de dióxido de carbono. Los RCP 4.5 y 6.0 son un escenario de emisiones intermedias de dióxido de carbono. El RCP 8.5 es aumento considerable en las emisiones de carbono.

El criterio de selección de las variables es en función del porcentaje de aporte de información por lo cual se realizo un test de correlación (Pearson $>0,75$ ), por lo cual de las 19 solo fueron utilizadas 4 variables bioclimáticas: bio1 (temperatura media anual), bio4 (estacionalidad en la temperatura), bio 7 (amplitud térmica) y bio12 (precipitación anual) a una resolución espacial de 5 arcominutos $\left(170 \mathrm{~km}^{2}\right)$.

El parámetro utilizado para evaluar la capacidad de predecir de los modelos generados por Maxent es la curva ROC que contempla al mismo tiempo el Área por Debajo de la Curva (AUC). También serán medidas la contribución de cada variable en los modelos predictivos. 


\section{Resultados}

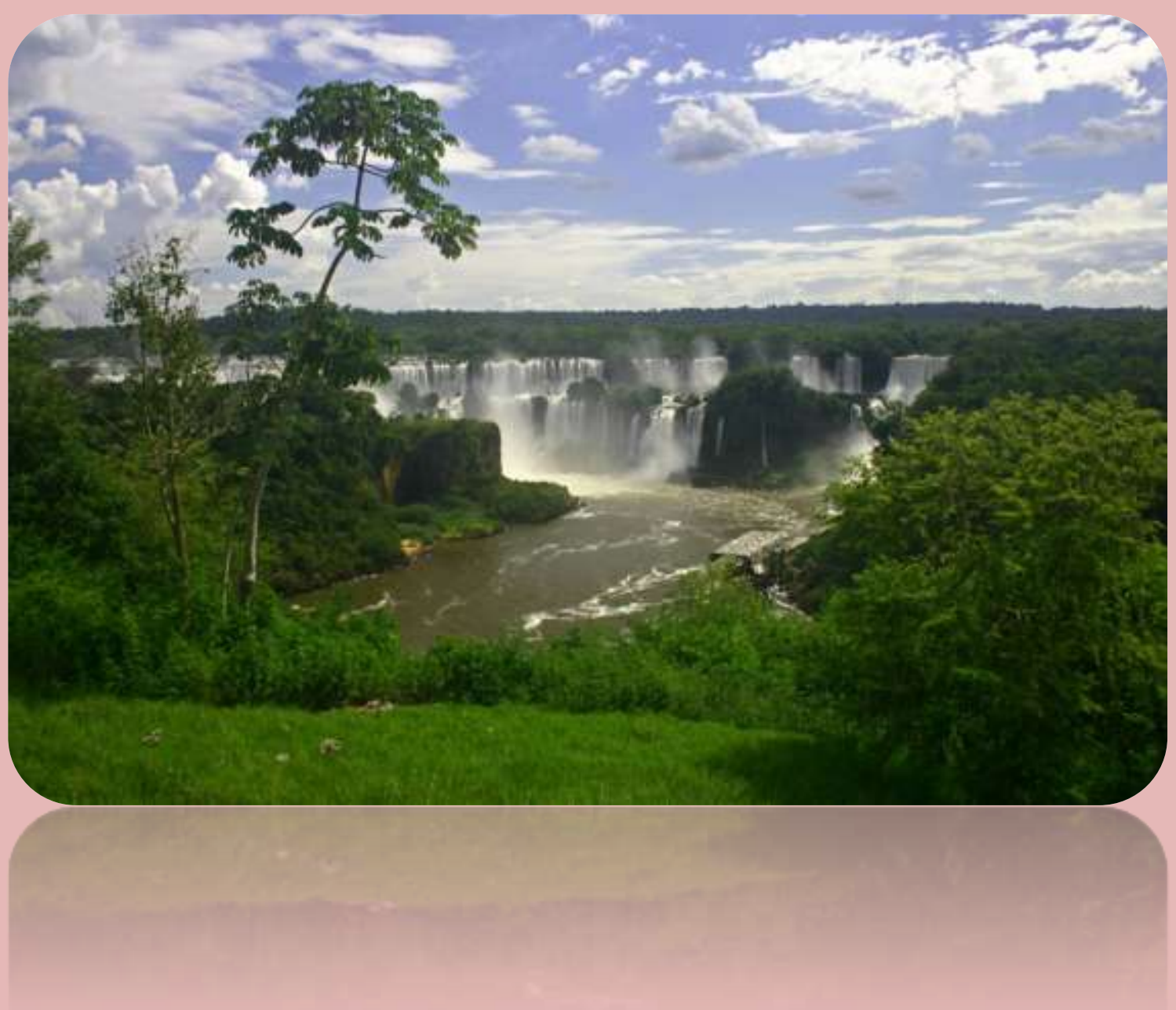




\section{Parte I: Los hospedadores RESULTADOS}

\subsection{Distribución temporal y espacial de los hospedadores}

Los resultados del análisis de la distribución temporal de las especies hospedadoras Lissachatina fulica, Phyllocaulis variegatus y Latipes erinaceus en la ciudad de Puerto Iguazú se observan en la figura 15. De las tres especies de moluscos incluidas en este trabajo, L. fulica fue la única que se halló en todas las campañas de muestreo y se observó que en los bimestres octubre-noviembre de 2013, febrero-marzo de 2014 y junio-julio de 2014, la cantidad de individuos recolectados fue similar (unidad de muestreo, 15'). A partir del muestreo octubre-noviembre de 2014, se visualizó un incremento en el número de individuos recolectados, lo cual evidencia un mayor número poblacional en cada uno de los sitios.

En lo que concierne a las babosas de la familia Veronicellidae: $P$. variegatus y $L$. erinaceus, se observó que la cantidad de ejemplares recolectados fue disminuyendo a lo largo de los bimestres, siendo el bimestre octubre-noviembre de 2013 en el que se pudieron capturar la mayor cantidad de ejemplares (Figura 15). La recolección de individuos fue decreciendo de junio-julio (2014) a junio-julio (2015).

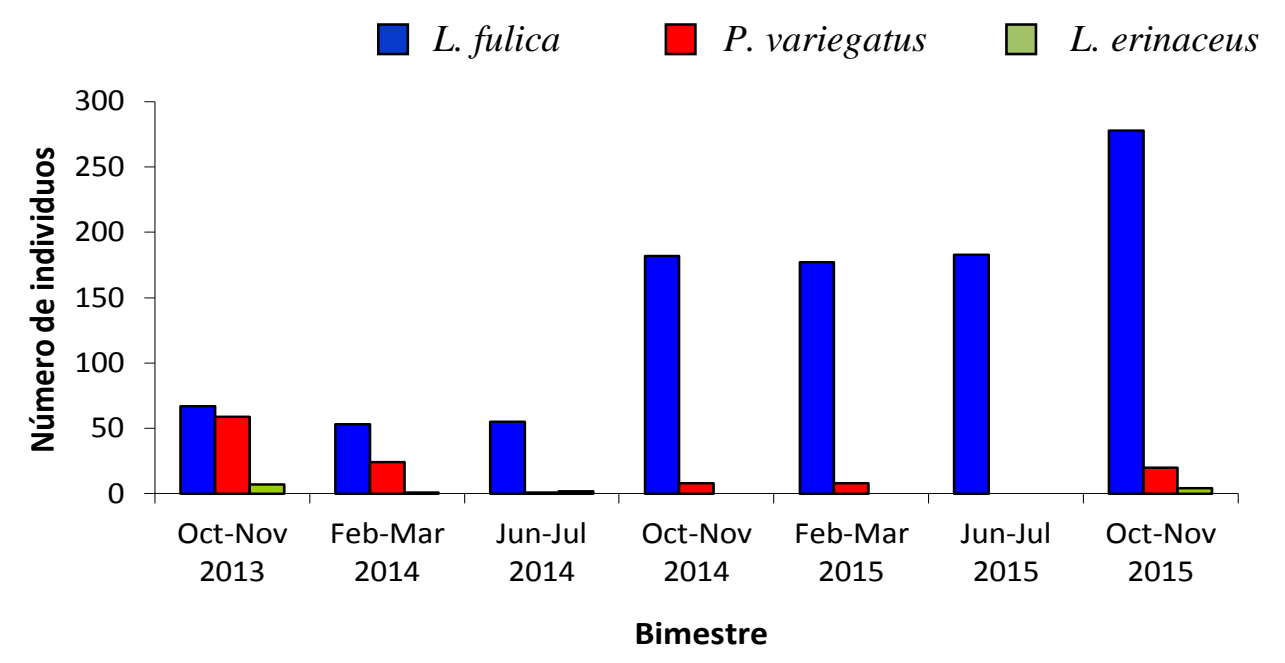

Figura 15. Distribución temporal de los hospedadores.

La distribución espacial de las tres especies hospedadoras se puede visualizar en las figuras 16-24. 
En el caso de L. fulica se observó que en los bimestres octubre-noviembre de 2013 y febrero-marzo de 2014 (Figura 16) los ejemplares sólo se hallaron en los lugares focos registrados inicialmente en el 2010 (i.e. barrio Ribera del Paraná, Villa Nueva, y área portuaria). Se observó que en menos de un año (Figura 17) L. fulica se dispersó hacia el interior del casco urbano, mientras que hacia fines de 2014 y durante el 2015 esta especie hospedadora logró dispersarse hacia otros barrios (Figura 18), llegando en noviembre del 2016 a todos los barrios de la ciudad de Puerto Iguazú (Valente, obs. pers.).

Por el contrario respecto a las babosas Veronicellidae, $P$. variegatus y $L$. erinaceus, se observó una reducción en su distribución espacial. En los muestreos de 2014 a 2015, disminuyó la cantidad de sitios de colecta efectiva de babosas, prevaleciendo en zonas más cercanas a los cursos de agua, lo cual indicaría una reducción en el número poblacional en el interior del casco urbano (Figuras 19-24).

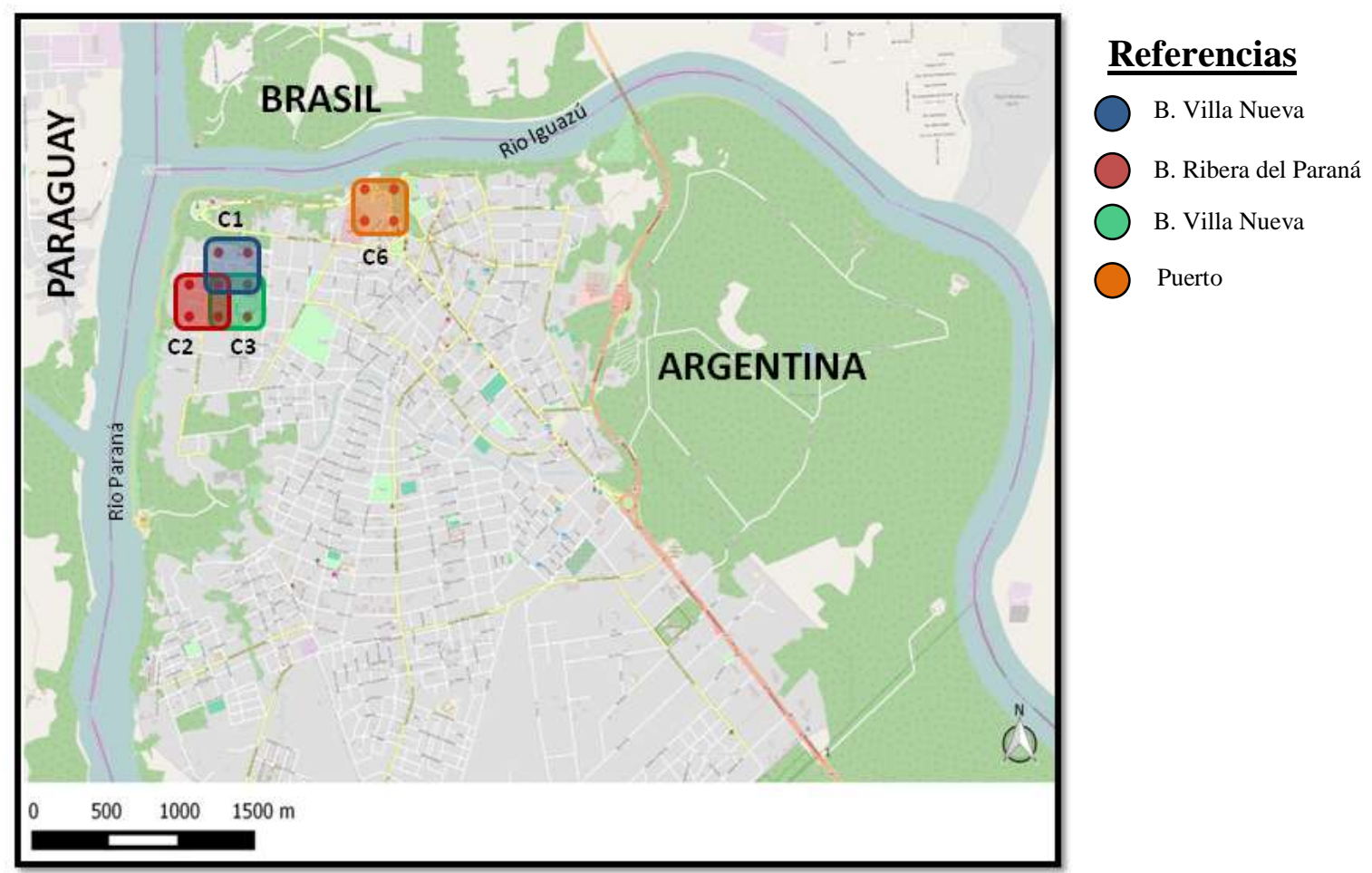

Figura 16. Mapa de distribución espacial durante el período octubre 2013-marzo 2014, en donde se encontró Lissachatina fulica sólo en las celdas: C1, C2, C3 y C6. 


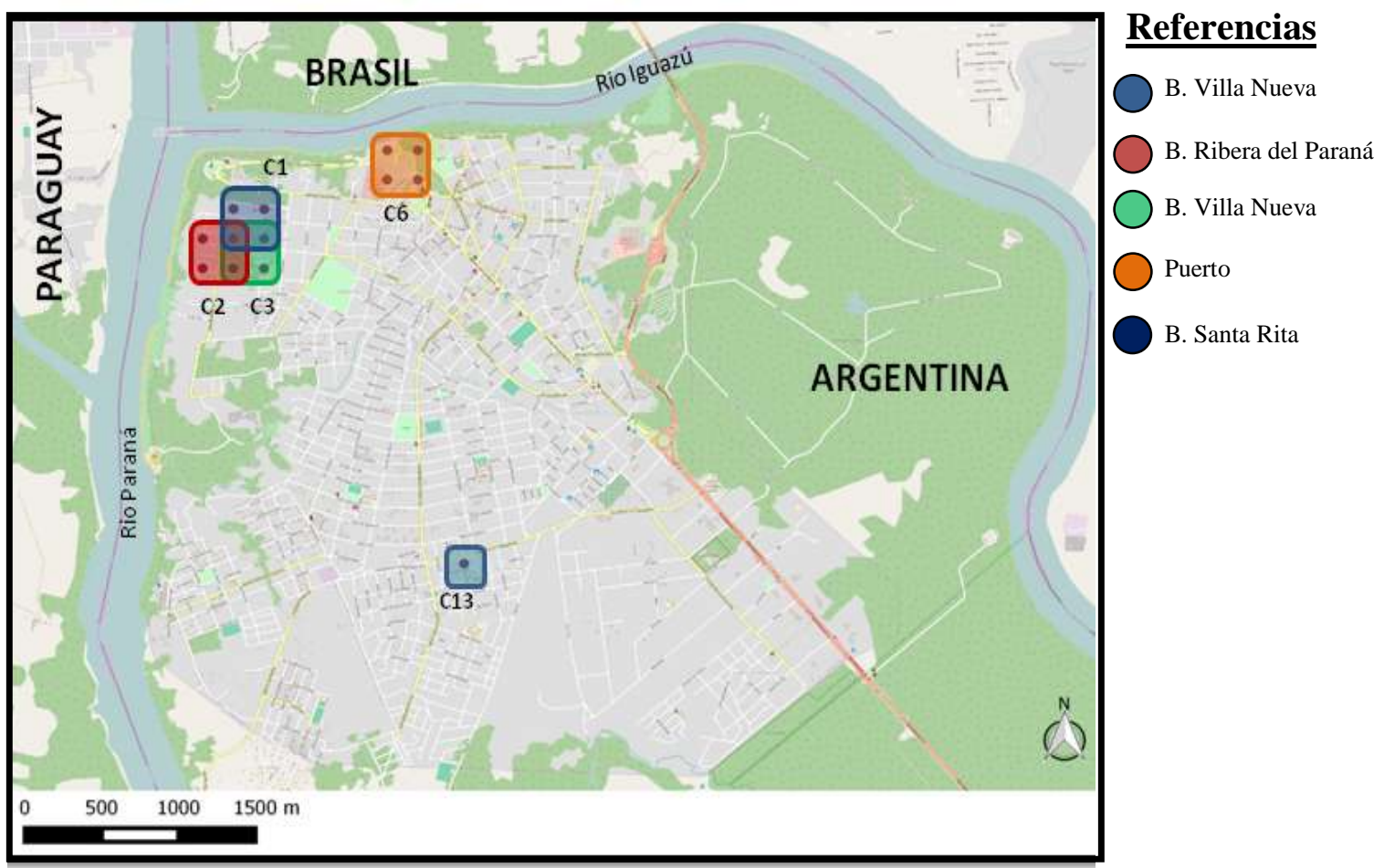

Figura 17. Mapa de distribución espacial durante el período junio-octubre 2014, en donde se encontró Lissachatina fulica sólo en las celdas: C1, C2, C3, C6 y C13.

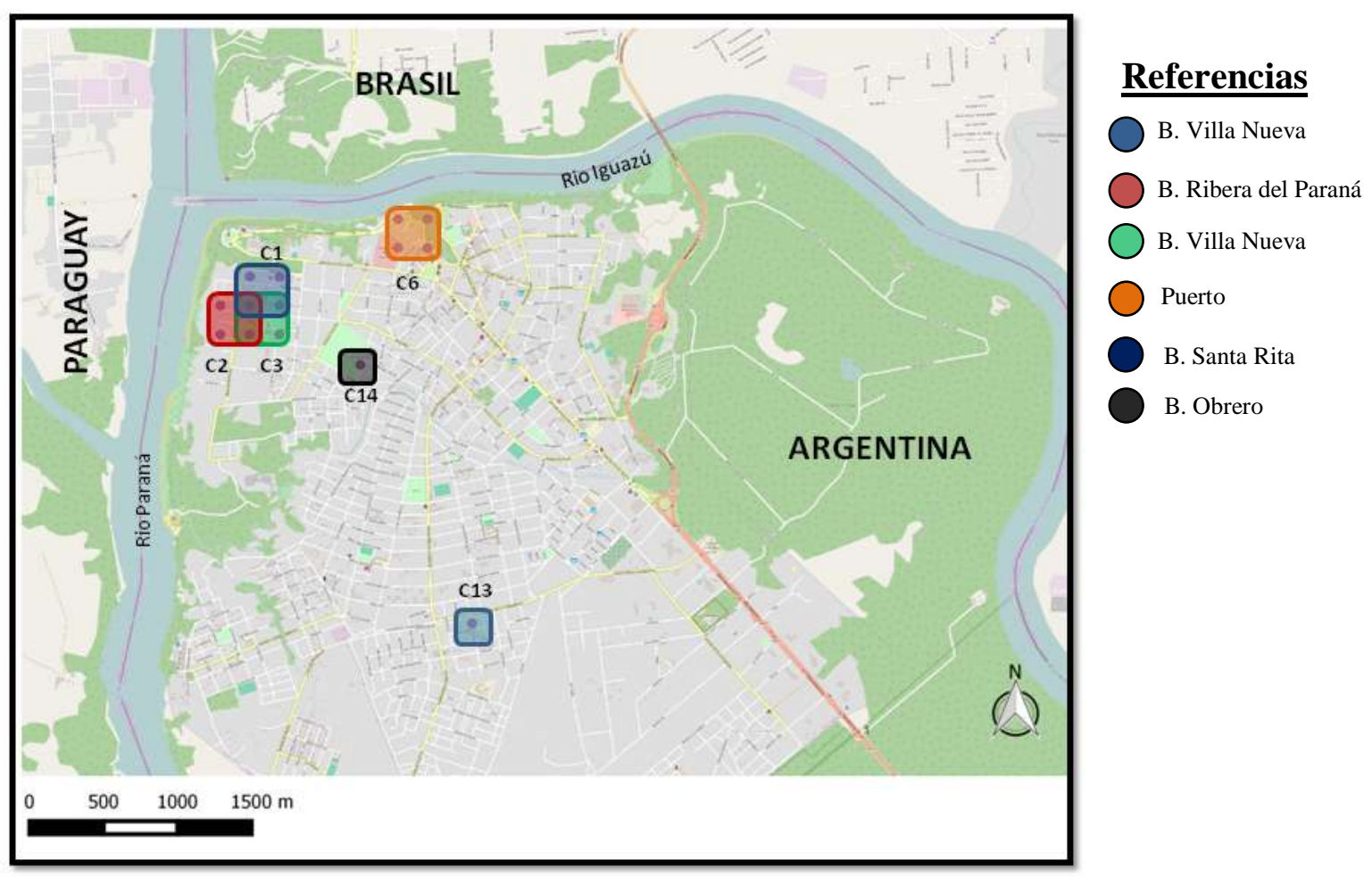

Figura 18. Mapa de distribución espacial durante el período noviembre 2014-noviembre 2015, en donde se encontró Lissachatina fulica sólo en las celdas: C1, C2, C3, C6, C13 y C14. 


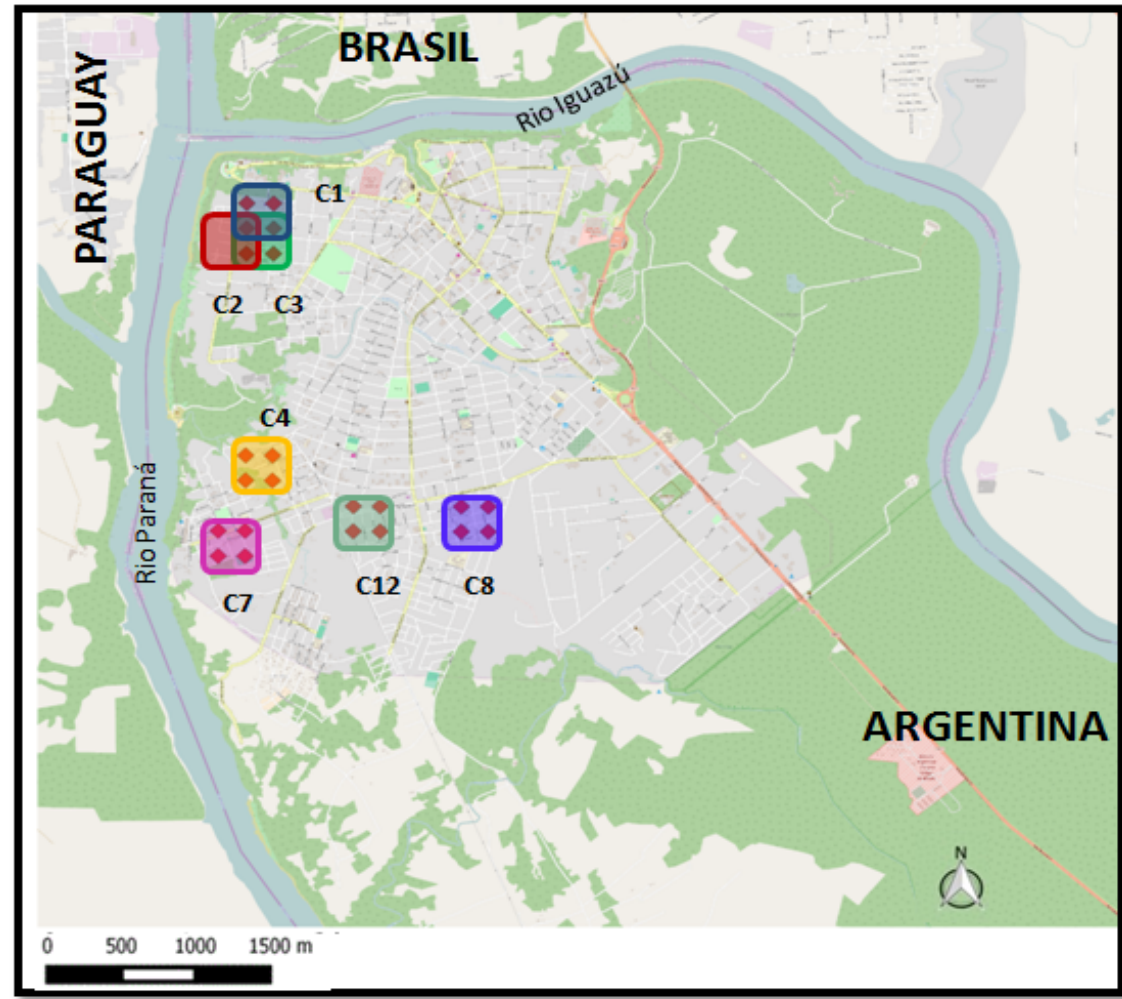

\section{Referencias}

B. Villa Nueva

B. Ribera del Paraná

$\bigcirc$ B. Villa Nueva

$\bigcirc$ B. Libertad

B. Santa Rosa

B. Santa Rita

B. Los Trabajadores

Figura 19. Mapa de distribución espacial durante el período octubre-noviembre 2013, en donde se encontró Phyllocaulis variegatus sólo en las celdas: C1, C2, C3, C4, C7, C8 y C12.

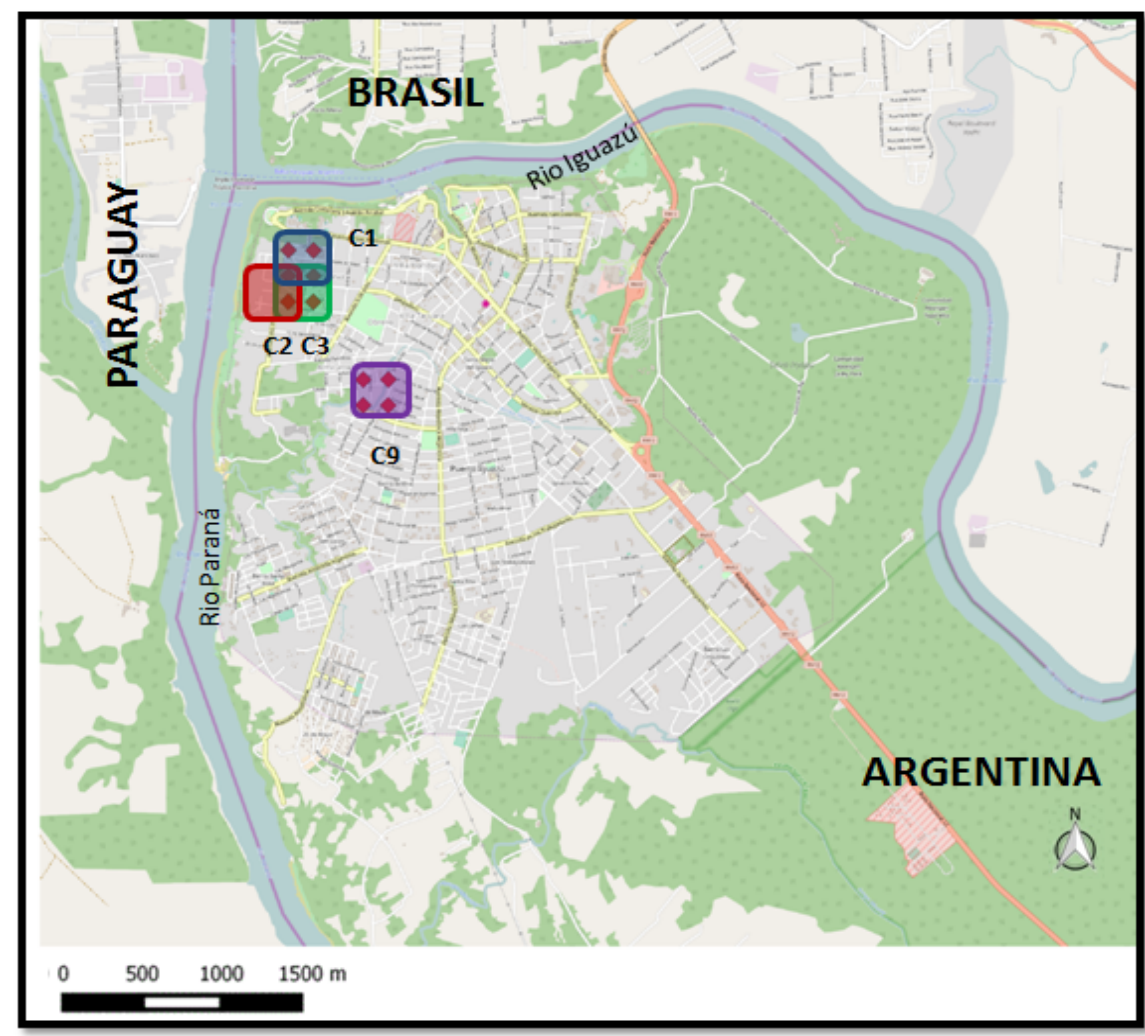

\section{$\underline{\text { Referencias }}$}

B. Villa Nueva

B. Ribera del Paraná

$\bigcirc$ B. Villa Nueva

B. Almirante Brown

Figura 20. Mapa de distribución espacial durante el período octubre-noviembre 2014, en donde se encontró Phyllocaulis variegatus sólo en las celdas: C1, C2 (únicamente en dos puntos), C3 y C9. 


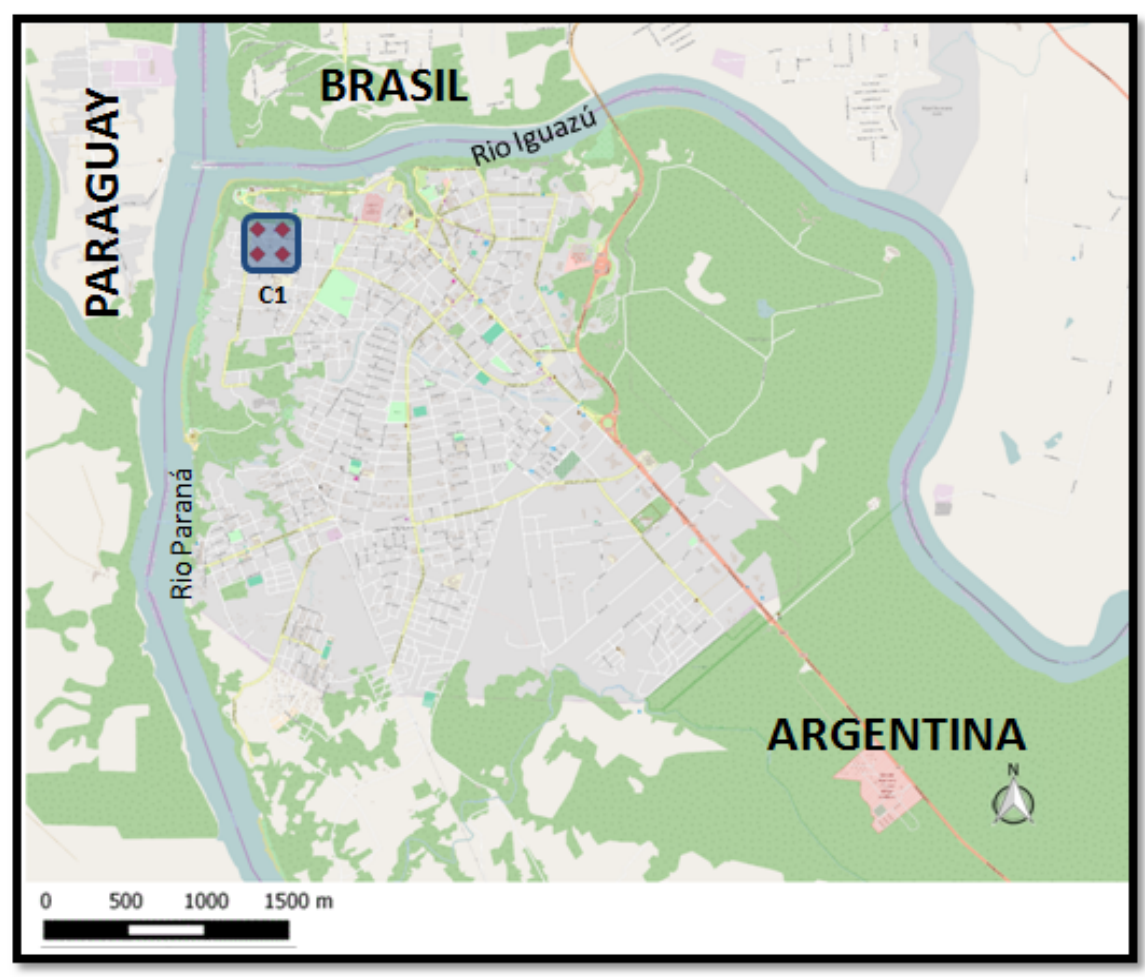

\section{$\underline{\text { Referencias }}$}

B. Villa Nueva

Figura 21. Mapa de distribución espacial durante el período octubre-noviembre 2015, en donde se encontró Phyllocaulis variegatus sólo en la celda: C1.

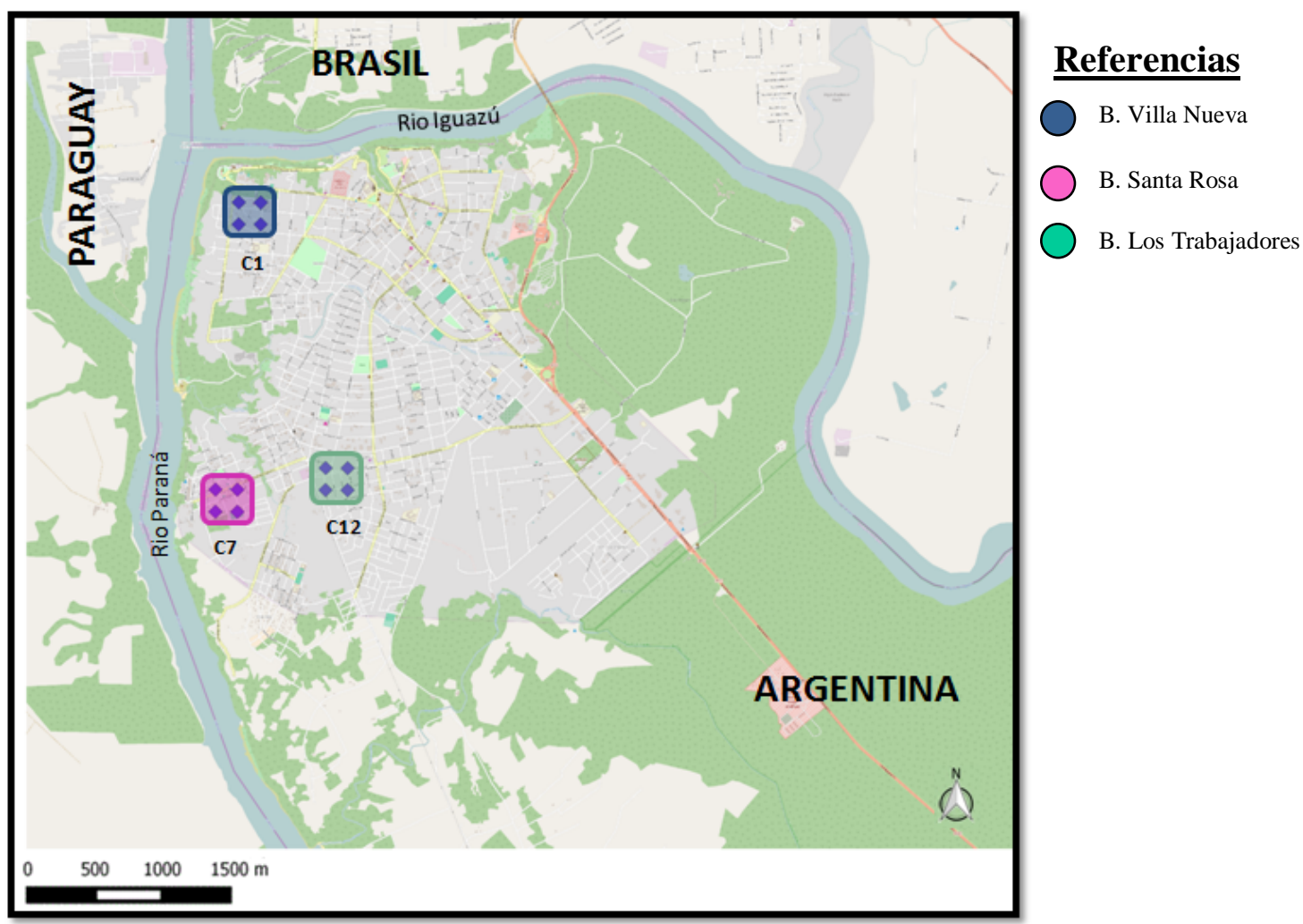

Figura 22. Mapa de distribución espacial de durante el período octubre-noviembre 2013, en donde se encontró Latipes erinaceus sólo en las celdas: C1, C7, y C12. 


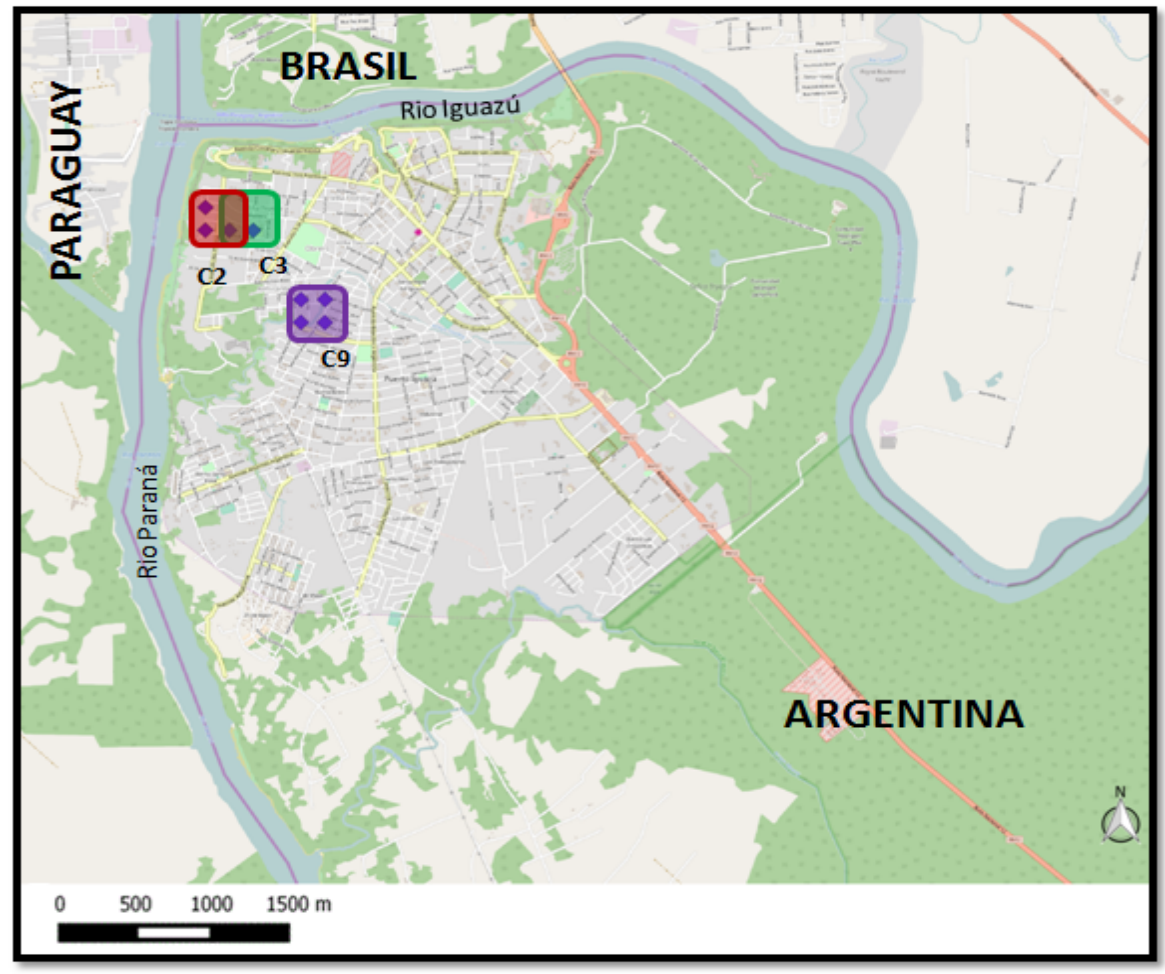

\section{$\underline{\text { Referencias }}$}

B. Ribera del Paraná

B. Villa Nueva

B. Almirante Brown

Figura 23. Mapa de distribución espacial de durante el período octubre-noviembre 2014, en donde se encontró Latipes erinaceus sólo en las celdas: C2, C3, y C9.

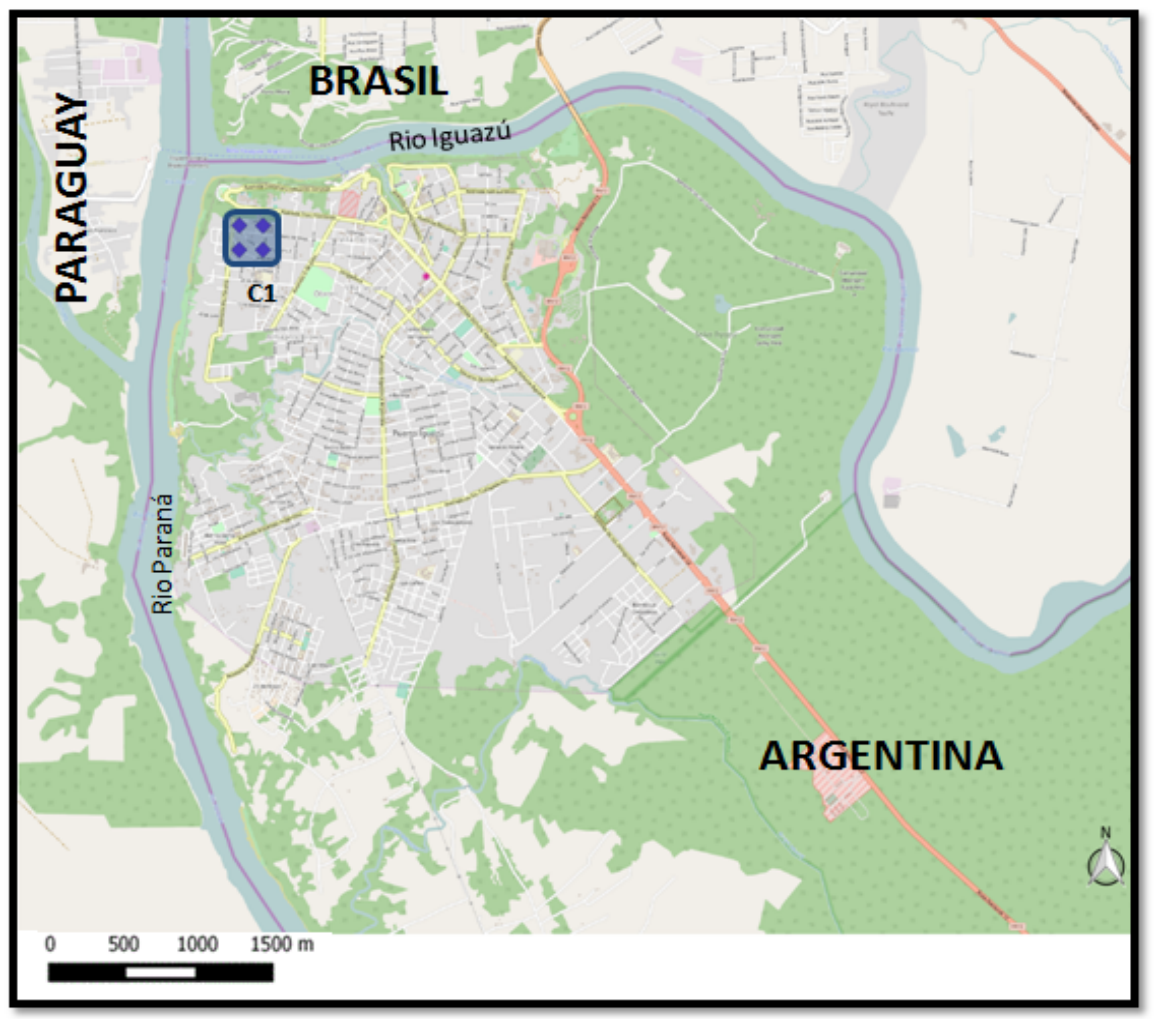

\section{$\underline{\text { Referencias }}$}

B. Villa Nueva

Figura 24. Mapa de distribución espacial de durante el período octubre-noviembre 2015, en donde se encontró Latipes erinaceus sólo en la celda: C1. 


\subsection{Distribución de talla de los hospedadores en función del tiempo y las variables}

\section{ambientales}

\section{Lissachatina fulica}

En la tabla 2 y figura 25 se presenta la distribución de tallas de L. fulica por bimestre. Se observa que en los bimestres octubre-noviembre de 2013 (O-N 2013), 2014 (O-N 2014) y 2015 (O-N 2015) las tallas mejor representadas son aquellas que corresponden al intervalo 2 [3-6 cm), en los bimestres febrero-marzo 2014 (F-M 2014) y 2015 (F-M 2015) las tallas que prevalecen son las que corresponden al intervalo 3 [6$9 \mathrm{~cm}$ ), mientras que en los bimestres junio-julio 2014 (J-J 2014) y 2015 (J-J 2015) no se observó una talla predominante (período de hibernación). Los análisis de correspondencia entre la distribución de tallas y los bimestres (Figura 26), indicaron que las tallas del intervalo 1 [0-3 cm), están asociadas a los bimestres junio-julio (J-J), las tallas intermedias del intervalo 2 [3-6 cm) están asociadas al bimestre octubrenoviembre $(\mathrm{O}-\mathrm{N})$, y las tallas más grandes (intervalos 3 y 4; [6-12 cm), están relacionadas al bimestre febrero-marzo (F-M).

Si se analiza la evolución de una población durante cada ciclo anual (tomando como inicio de cada ciclo el período post-hibernación: posterior al bimestre junio-julio) se observa que la mayor frecuencia de individuos de tallas mayores [6-9 $\mathrm{cm}$ ) se encuentra en los bimestres febrero-marzo. 
Tabla 2. Distribución de tallas de Lissachatina fulica en cada bimestre.

\begin{tabular}{|c|c|c|c|c|c|c|c|c|}
\hline $\begin{array}{l}\text { Intervalos } \\
\text { de tamaño }\end{array}$ & $\begin{array}{c}\text { Oct-Nov } \\
2013\end{array}$ & $\begin{array}{c}\text { Feb-Mar } \\
2014\end{array}$ & $\begin{array}{c}\text { Jun-Jul } \\
2014\end{array}$ & $\begin{array}{c}\text { Oct-Nov } \\
2014\end{array}$ & $\begin{array}{c}\text { Feb-Mar } \\
2015\end{array}$ & $\begin{array}{c}\text { Jun-Jul } \\
2015\end{array}$ & $\begin{array}{l}\text { Oct-Nov } \\
2015\end{array}$ & SubTotal \\
\hline \multirow{2}{*}{$\begin{array}{c}1 \\
{[0-3 \mathrm{~cm})}\end{array}$} & $\mathbf{n}=11$ & $\mathbf{n}=8$ & $\mathbf{n}=17$ & $\mathbf{n}=39$ & $\mathbf{n}=19$ & $n=65$ & $\mathbf{n}=6$ & $N=165$ \\
\hline & $\mathbf{p}=0,01$ & $\mathbf{p}=0,008$ & $\mathbf{p}=0,017$ & $\mathbf{p}=0,039$ & $\mathbf{p}=0,019$ & $\mathbf{p}=0,065$ & $\mathbf{p}=0,006$ & $P=0,165$ \\
\hline \multirow{2}{*}{$\begin{array}{c}2 \\
{[3-6 \mathrm{~cm})}\end{array}$} & $\mathbf{n}=37$ & $\mathbf{n}=10$ & $\mathbf{n}=15$ & $\mathbf{n}=90$ & $\mathbf{n}=53$ & $\mathbf{n}=68$ & $\mathbf{n}=217$ & $\mathrm{~N}=490$ \\
\hline & $\mathbf{p}=0,03$ & $\mathbf{p}=0,010$ & $\mathbf{p}=0,015$ & $\mathbf{p}=0,09$ & $\mathbf{p}=0,053$ & $\mathbf{p}=0,068$ & $\mathbf{p}=0,218$ & $P=0,492$ \\
\hline \multirow{2}{*}{$\begin{array}{c}3 \\
{[6-9 \mathrm{~cm})}\end{array}$} & $\mathbf{n}=18$ & $\mathbf{n}=27$ & $\mathbf{n}=22$ & $\mathbf{n}=51$ & $\mathbf{n}=101$ & $\mathbf{n}=49$ & $\mathbf{n}=51$ & $\mathrm{~N}=\mathbf{3 1 9}$ \\
\hline & $\mathbf{p}=0,01$ & $\mathbf{p}=0,027$ & $\mathbf{p}=0,022$ & $\mathbf{p}=0,051$ & $\mathbf{p}=0,101$ & $\mathbf{p}=0,049$ & $\mathbf{p}=0,051$ & $P=0,32$ \\
\hline \multirow{2}{*}{$\begin{array}{c}4 \\
{[9-12 \mathrm{~cm})}\end{array}$} & $\mathbf{n}=1$ & $\mathbf{n}=8$ & $\mathbf{n}=1$ & $\mathbf{n}=2$ & $\mathbf{n}=4$ & $\mathbf{n}=1$ & $\mathbf{n}=4$ & $\mathrm{~N}=21$ \\
\hline & $\mathbf{p}=0,001$ & $\mathbf{p}=0,008$ & $\mathbf{p}=0,001$ & $\mathbf{p}=0,002$ & $\mathbf{p}=0,004$ & $\mathbf{p}=0,001$ & $\mathbf{p}=0,004$ & $P=0,021$ \\
\hline \multirow{2}{*}{ Subtotal } & $N=67$ & $N=53$ & $N=55$ & $\mathrm{~N}=182$ & $\mathbf{N}=177$ & $N=183$ & $N=278$ & \multirow{2}{*}{$\begin{array}{c}\text { TOTAL } \\
995\end{array}$} \\
\hline & $P=0,06$ & $P=0,053$ & $P=0,055$ & $P=0,182$ & $P=0,177$ & $P=0,183$ & $P=0,278$ & \\
\hline
\end{tabular}

$\mathbf{p}=$ proporción de cada celda

$\mathbf{P}=$ Subtotal de proporción

$\mathbf{n}=$ número de ejemplares

$\mathbf{N}=$ subtotal de número de ejemplares 


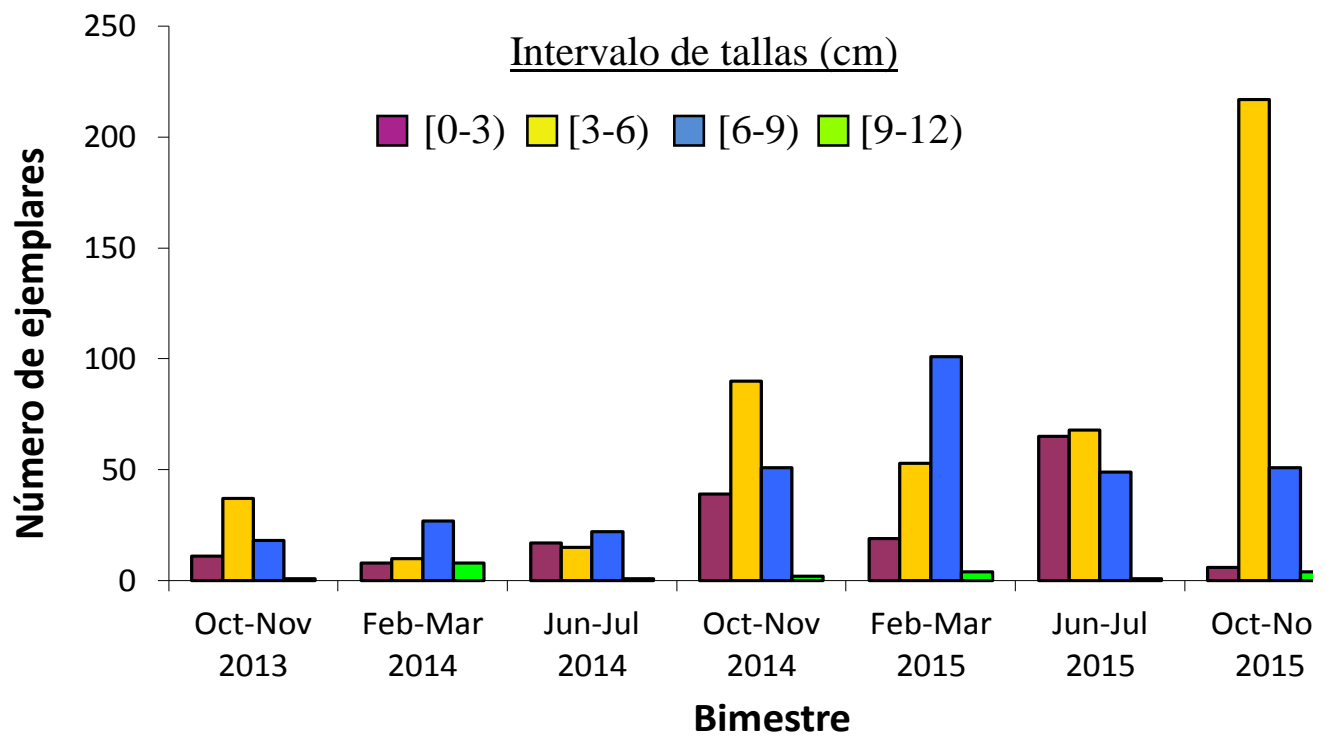

Figura 25. Distribución de tallas de Lissachatina fulica.

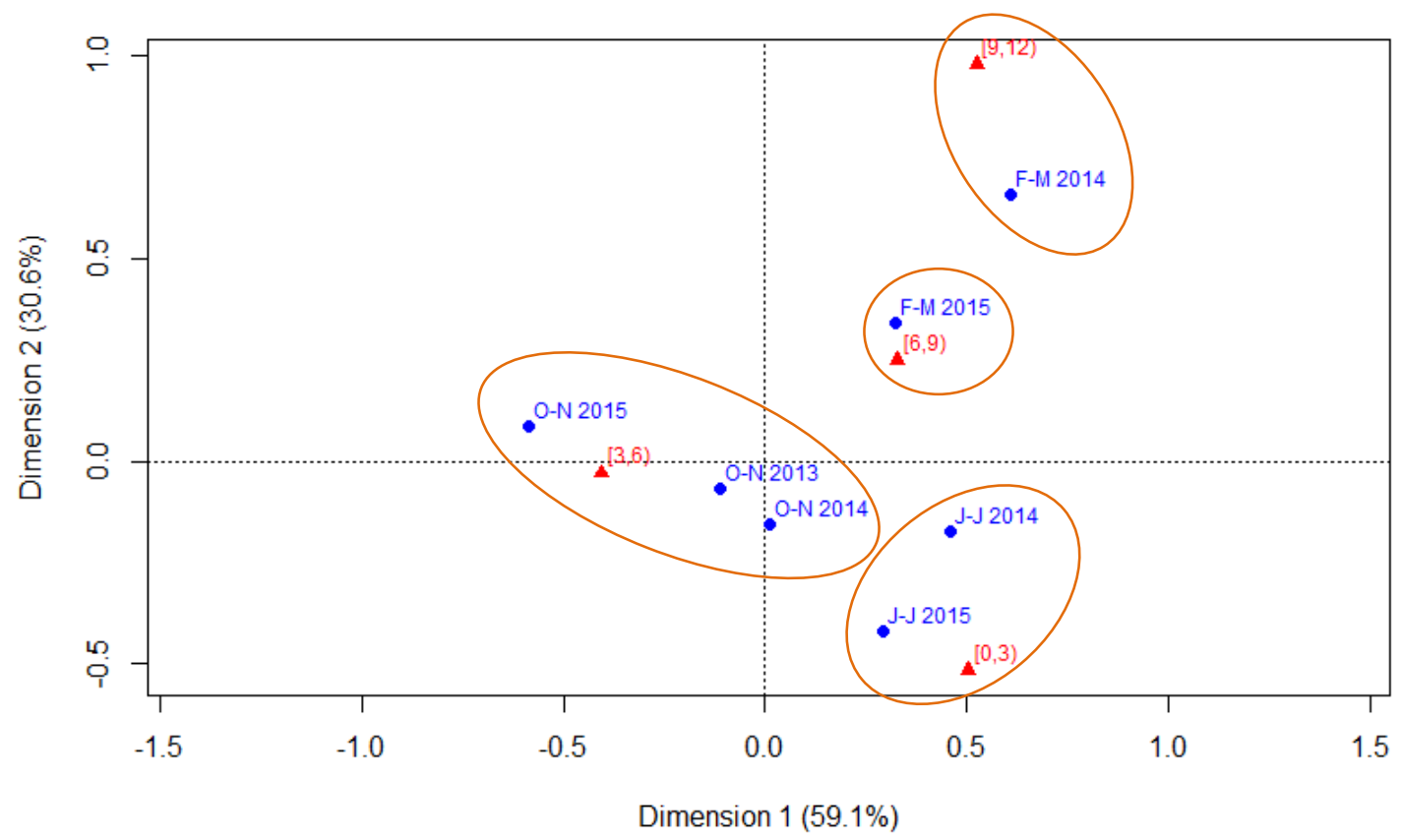

Figura 26. Análisis de correspondencia para Lissachatina fulica. 


\section{Babosas Veronicellidae}

En relación a las babosas Veronicellidae se observó que las tallas intermedias, intervalos 2 y 3 [3-9 cm), fueron las más abundantes a lo largo de todos los bimestres (Tablas 3-4).

Para el caso particular de $P$. variegatus la figura 27 muestra la distribución de tallas en cada bimestre, en concordancia con lo mencionado anteriormente. El análisis de correspondencia entre la distribución de tallas y los bimestres (Figura 28) indica una fuerte asociación entre el intervalo de talla 2 y el bimestre de octubre-noviembre 2013 (O-N 2013), y entre el intervalo 3 y el bimestre octubre-noviembre 2015 (O-N 2015), constatando que en esos bimestres las tallas predominantes fueron la 2 y la 3.

En lo que se refiere a L. erinaceus en la figura 29 se puede observar la distribución de tallas en cada bimestre. El análisis de correspondencia para esta especie no pudo realizarse por la ausencia de ejemplares en algunos bimestres (octubrenoviembre 2014, febrero-marzo 2015, junio-julio 2015). 
Tabla 3. Distribución de tallas de Phyllocaulis variegatus en cada bimestre.

\begin{tabular}{|c|c|c|c|c|c|c|c|c|}
\hline $\begin{array}{l}\text { Intervalos } \\
\text { de tamaño }\end{array}$ & $\begin{array}{c}\text { Oct-Nov } \\
2013\end{array}$ & $\begin{array}{c}\text { Feb-Mar } \\
2014\end{array}$ & $\begin{array}{c}\text { Jun-Jul } \\
2014\end{array}$ & $\begin{array}{c}\text { Oct-Nov } \\
2014\end{array}$ & $\begin{array}{c}\text { Feb-Mar } \\
2015\end{array}$ & $\begin{array}{c}\text { Jun-Jul } \\
2015\end{array}$ & $\begin{array}{c}\text { Oct-Nov } \\
2015\end{array}$ & SubTotal \\
\hline \multirow{2}{*}{$\begin{array}{c}1 \\
{[0-3 \mathrm{~cm})}\end{array}$} & $\mathbf{n}=3$ & $\mathbf{n}=0$ & $\mathbf{n}=0$ & $\mathbf{n}=2$ & $\mathbf{n}=0$ & $\mathbf{n}=0$ & $\mathbf{n}=0$ & $N=5$ \\
\hline & $\mathbf{p}=0,025$ & $\mathbf{p}=0$ & $\mathbf{p}=0$ & $\mathbf{p}=0,016$ & $\mathbf{p}=0$ & $\mathbf{p}=0$ & $\mathbf{p}=0$ & $P=0,04$ \\
\hline \multirow{2}{*}{$\begin{array}{c}2 \\
{[3-6 \mathrm{~cm})}\end{array}$} & $\mathbf{n}=38$ & $\mathbf{n}=7$ & $\mathbf{n}=0$ & $\mathbf{n}=4$ & $\mathbf{n}=6$ & $\mathbf{n}=0$ & $\mathbf{n}=6$ & $N=61$ \\
\hline & $\mathbf{p}=0,31$ & $\mathbf{p}=0,058$ & $\mathbf{p}=0$ & $\mathbf{p}=0,033$ & $\mathbf{p}=0,05$ & $\mathbf{p}=0$ & $\mathbf{p}=0,05$ & $P=0,5$ \\
\hline \multirow{2}{*}{$\begin{array}{c}3 \\
{[6-9 \mathrm{~cm})}\end{array}$} & $\mathbf{n}=17$ & $\mathbf{n}=15$ & $\mathbf{n}=1$ & $\mathbf{n}=1$ & $\mathbf{n}=2$ & $\mathbf{n}=0$ & $\mathbf{n}=13$ & $N=49$ \\
\hline & $\mathbf{p}=0,14$ & $\mathbf{p}=0,125$ & $\mathbf{p}=0,008$ & $\mathbf{p}=0,008$ & $\mathbf{p}=0,016$ & $\mathbf{p}=0$ & $\mathbf{p}=0,10$ & $P=0,4$ \\
\hline \multirow{2}{*}{$\begin{array}{c}4 \\
{[9-12 \mathrm{~cm})}\end{array}$} & $\mathbf{n}=1$ & $\mathbf{n}=2$ & $\mathbf{n}=0$ & $\mathbf{n}=1$ & $\mathbf{n}=0$ & $\mathbf{n}=0$ & $\mathbf{n}=1$ & $N=5$ \\
\hline & $\mathbf{p}=0,008$ & $\mathbf{p}=0,016$ & $\mathbf{p}=0$ & $\mathbf{p}=0,008$ & $\mathbf{p}=0$ & $\mathbf{p}=0$ & $\mathbf{p}=0,008$ & $P=0,04$ \\
\hline \multirow{2}{*}{ Subtotal } & $N=59$ & $N=24$ & $N=1$ & $\mathbf{N}=8$ & $\mathbf{N}=8$ & $\mathbf{N}=\mathbf{0}$ & $\mathrm{N}=\mathbf{2 0}$ & \multirow{2}{*}{$\begin{array}{c}\text { TOTAL } \\
\mathbf{1 2 0}\end{array}$} \\
\hline & $P=0,49$ & $P=0,2$ & $P=0,008$ & $P=0,066$ & $P=0,066$ & $\mathbf{P}=\mathbf{0}$ & $P=0,16$ & \\
\hline
\end{tabular}

$\mathbf{p}=$ proporción de cada celda

$\mathbf{P}=$ Subtotal de proporción

$\mathbf{n}=$ número de ejemplares

$\mathbf{N}=$ subtotal de número de ejemplares 
Tabla 4. Distribución de tallas de Latipes erinaceus en cada bimestre.

\begin{tabular}{|c|c|c|c|c|c|c|c|c|}
\hline $\begin{array}{l}\text { Intervalos } \\
\text { de tamaño }\end{array}$ & $\begin{array}{c}\text { Oct-Nov } \\
2013\end{array}$ & $\begin{array}{c}\text { Feb-Mar } \\
2014\end{array}$ & $\begin{array}{c}\text { Jun-Jul } \\
2014\end{array}$ & $\begin{array}{c}\text { Oct-Nov } \\
2014\end{array}$ & $\begin{array}{c}\text { Feb-Mar } \\
2015\end{array}$ & $\begin{array}{c}\text { Jun-Jul } \\
2015\end{array}$ & $\begin{array}{c}\text { Oct-Nov } \\
2015\end{array}$ & SubTotal \\
\hline \multirow{2}{*}{$\begin{array}{c}1 \\
{[0-3 \mathrm{~cm})}\end{array}$} & $\mathbf{n}=0$ & $\mathbf{n}=0$ & $\mathbf{n}=0$ & $\mathbf{n}=0$ & $\mathbf{n}=0$ & $\mathbf{n}=0$ & $\mathbf{n}=0$ & $\mathbf{N}=\mathbf{0}$ \\
\hline & $\mathbf{p}=0$ & $\mathbf{p}=0$ & $\mathbf{p}=0$ & $\mathbf{p}=0$ & $\mathbf{p}=0$ & $\mathbf{p}=0$ & $\mathbf{p}=0$ & $P=0$ \\
\hline \multirow{2}{*}{$\begin{array}{c}2 \\
{[3-6 \mathrm{~cm})}\end{array}$} & $\mathbf{n}=4$ & $\mathbf{n}=0$ & $\mathbf{n}=2$ & $\mathbf{n}=0$ & $\mathbf{n}=0$ & $\mathbf{n}=0$ & $\mathbf{n}=4$ & $N=10$ \\
\hline & $\mathbf{p}=0,28$ & $\mathbf{p}=0$ & $\mathbf{p}=0,14$ & $\mathbf{p}=0$ & $\mathbf{p}=0$ & $\mathbf{p}=0$ & $\mathbf{p}=0,28$ & $P=0,71$ \\
\hline \multirow{2}{*}{$\begin{array}{c}3 \\
{[6-9 \mathrm{~cm})}\end{array}$} & $\mathbf{n}=3$ & $\mathbf{n}=1$ & $\mathbf{n}=0$ & $\mathbf{n}=0$ & $\mathbf{n}=0$ & $\mathbf{n}=0$ & $\mathbf{n}=0$ & $N=4$ \\
\hline & $\mathbf{p}=0,21$ & $\mathbf{p}=0,071$ & $\mathbf{p}=0$ & $\mathbf{p}=0$ & $\mathbf{p}=0$ & $\mathbf{p}=0$ & $\mathbf{p}=0$ & $P=0,28$ \\
\hline \multirow{2}{*}{$\begin{array}{c}4 \\
{[9-12 \mathrm{~cm})}\end{array}$} & $\mathbf{n}=0$ & $\mathbf{n}=0$ & $\mathbf{n}=0$ & $\mathbf{n}=0$ & $\mathbf{n}=0$ & $\mathbf{n}=0$ & $\mathbf{n}=0$ & $\mathbf{N}=\mathbf{0}$ \\
\hline & $\mathbf{p}=0$ & $\mathbf{p}=0$ & $\mathbf{p}=0$ & $\mathbf{p}=0$ & $\mathbf{p}=0$ & $\mathbf{p}=0$ & $\mathbf{p}=0$ & $\mathbf{P}=\mathbf{0}$ \\
\hline \multirow{2}{*}{ Subtotal } & $N=7$ & $\mathbf{N}=1$ & $\mathrm{~N}=\mathbf{2}$ & $\mathbf{N}=\mathbf{0}$ & $\mathbf{N}=\mathbf{0}$ & $\mathbf{N}=\mathbf{0}$ & $N=4$ & \multirow{2}{*}{$\begin{array}{c}\text { TOTAL } \\
\mathbf{1 4}\end{array}$} \\
\hline & $P=0,45$ & $P=0,071$ & $P=0,14$ & $P=0$ & $P=0$ & $P=0$ & $P=0,28$ & \\
\hline
\end{tabular}

$\mathbf{p}=$ proporción de cada celda

$\mathbf{P}=$ Subtotal de proporción

$\mathbf{n}=$ número de ejemplares

$\mathbf{N}=$ subtotal de número de ejemplares 


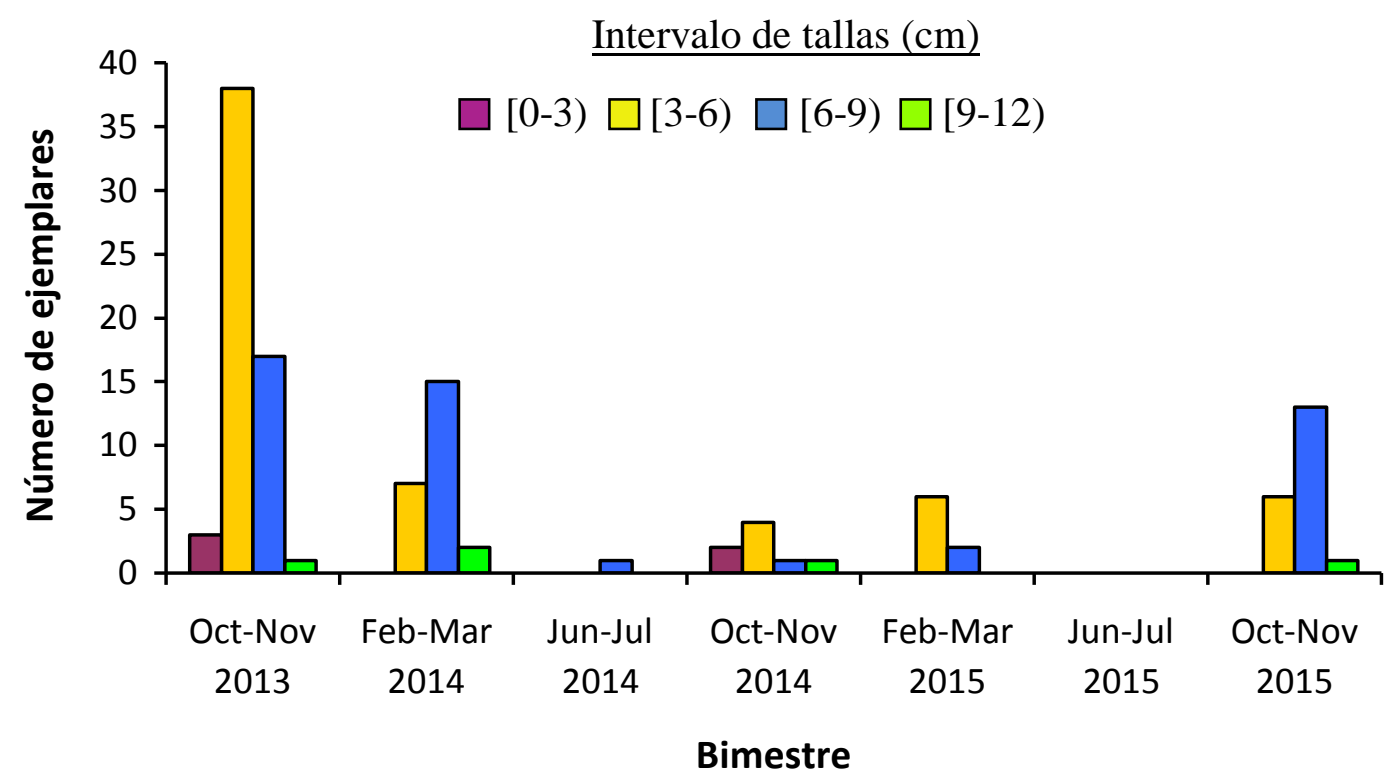

Figura 27. Distribución de tallas de Phyllocaulis variegatus.

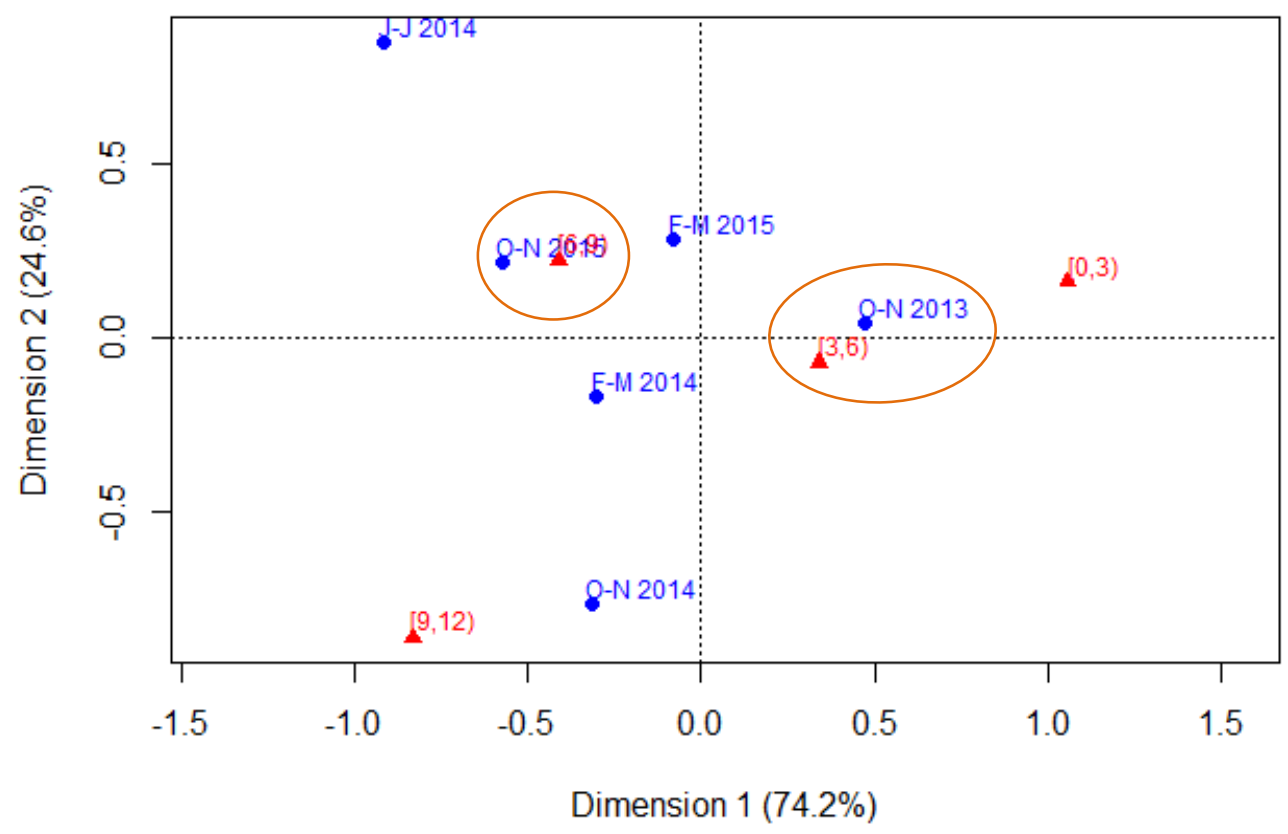

Figura 28. Análisis de correspondencia para Phyllocaulis variegatus. 


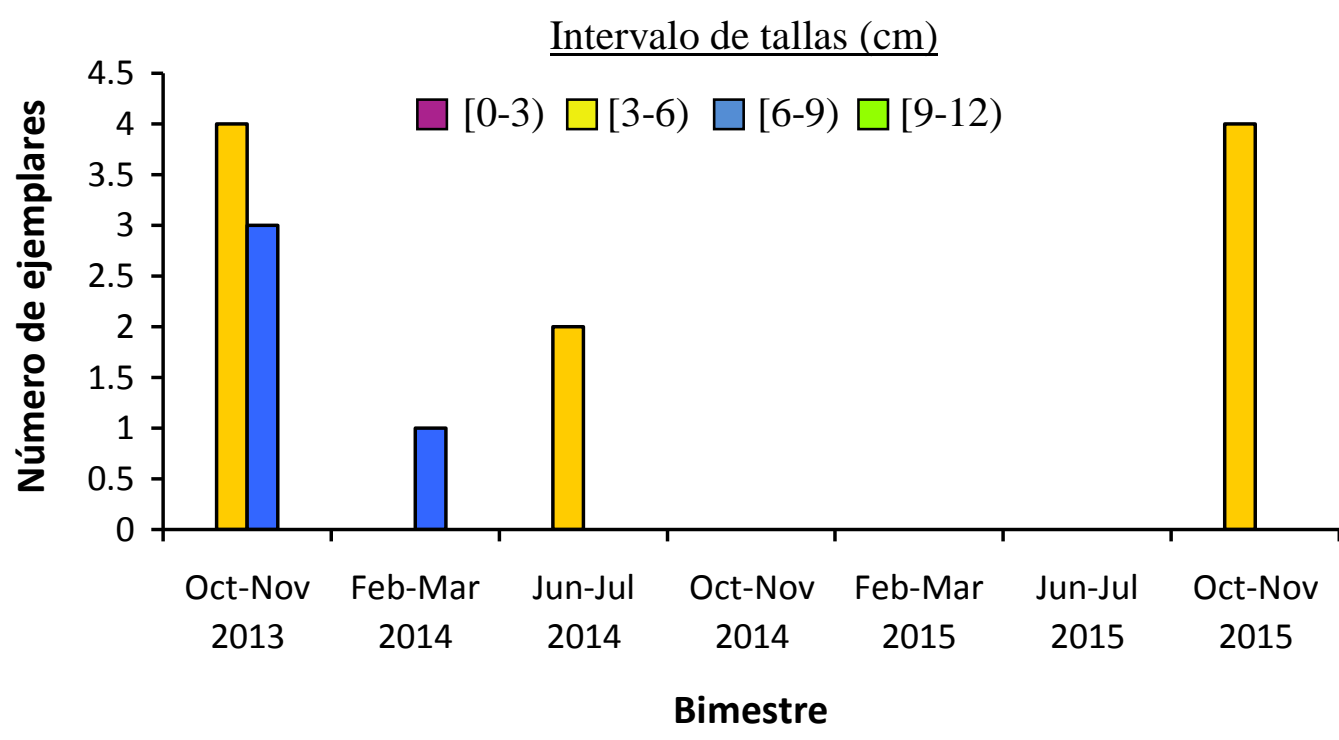

Figura 29. Distribución de tallas de Latipes erinaceus.

\subsection{Mapas predictivos de distribución de nichos ecológicos de L. fulica}

Los resultados obtenidos en los 4 escenarios o modelos de clima global se observan en la Figura 30.

El valor del AUC (valor por debajo de la curva) de los modelos de distribución geográfica potencial de $L$. fulica fue de 0.8 , predicen con buena probabilidad la distribución a futuro. Por lo cual los modelos predictivos de distribución obtenidos para L. fulica tuvieron un buen desempeño y se pueden considerar como robustos

De las cuatro variables bioclimáticas elegidas, la que aporta mayor porcentaje a los modelos y la de mayor contribución por la prueba de Jackknife es la variable bio 4, y en menor medida la variable bio7 (Figura 31).

En los 4 mapas se visualizan diferentes colores que representan la susceptibilidad de invasión de esta especie hospedadora en un área determinada (rojo=alta, naranja $=$ media alta, amarilla $=$ media, verde claro=media baja, verde oscuro=baja).

Para los modelos de nicho ecológico la estacionalidad de la temperatura (equivalente a humedad) y el rango anual de temperatura (temperatura máxima del mes más cálido- temperatura mínima del mes más frio) serian las variables que condicionan a futuro la distribución de L. fulica. 

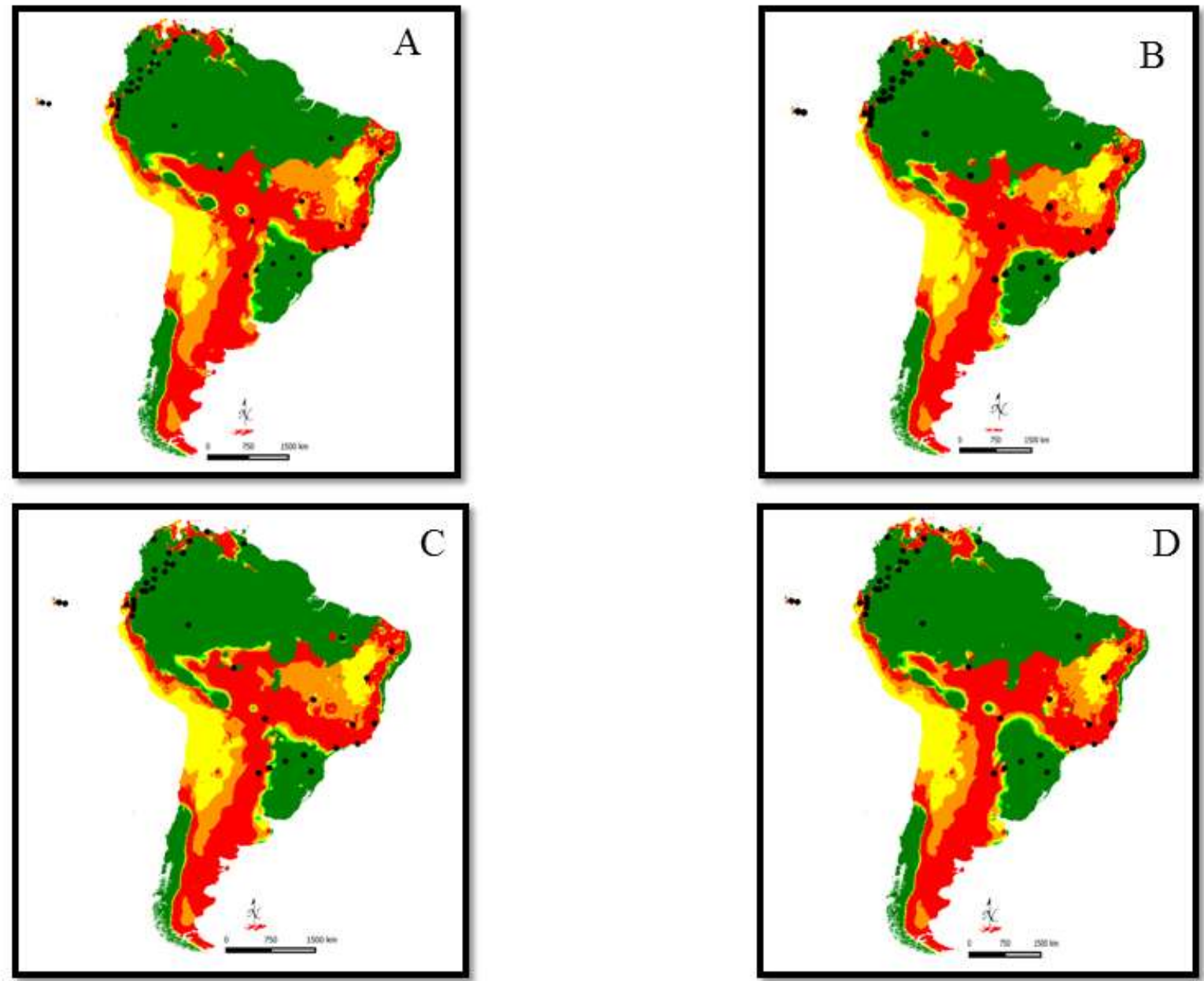

\section{Referencias}

Susceptibilidad de invasión

$\square$ Alta
$\square$ Media alta
$\square$ Media
$\square$ Media baja
$\square$ Baia

- Puntos de presencia de L. fulica

Figura 30. Mapas de distribución de nicho ecológicos de Lissachatina fulica en el 2050. A) Modelo de clima global menos drástico.; B -C) Modelo de clima global intermedio; D) Modelo de clima global más drástico. 


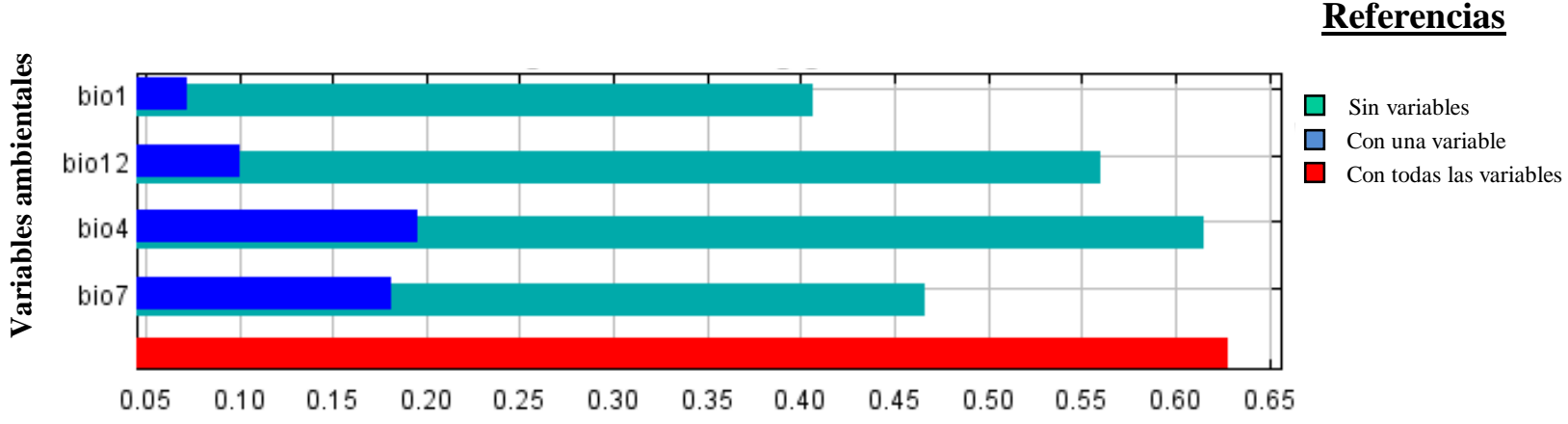

Figura 31. Porcentaje de aporte de información de cada variable.

\section{DISCUSIÓN}

\section{Distribución espacial y temporal de los hospedadores}

El área de distribución de una especie puede ser un concepto difícil de definir ya que involucra procesos y/o patrones verdaderamente complejos. Por ejemplo, toda distribución experimenta contracción y/o expansión espacial a lo largo del tiempo, dinámica influenciada por la interacción de factores biológicos, ecológicos y biogeográficos (Zunino \& Zullini, 2003). La forma en que se distribuye una especie o población en un espacio geográfico está determinada por factores históricos, ecológicos y fisiológicos que varían a lo largo de su distribución, sometiendo a las especies a diferentes condiciones bióticas y abióticas (Maciel-Mata et al., 2015). Puesto que la distribución espacial es un fenómeno dinámico, resulta difícil calcular con exactitud el área de distribución de una especie, más aún cuando se tratan de especies exóticas invasoras, que se caracterizan por su plasticidad adaptativa.

La distribución de las especies a escala regional puede estar influenciada, principalmente por factores climáticos. Sin embargo, la distribución de especies a escala regional no puede entenderse correctamente si no se tienen en cuenta una serie de circunstancias y elementos capaces de alterarla a escala local, tales como factores geomorfológicos, edáficos, hidrológicos y humanos (Maciel-Mata et al., 2015).

Una de las principales causas de la modificación en la distribución de las especies a nivel local es la introducción de nuevas especies a partir de la acción del hombre. Entre otras alteraciones, las especies exóticas causan desequilibrio en las interacciones de las redes tróficas, a menudo consumen o se alimentan de las nativas, se multiplican más rápidamente, las infectan o las convierten en portadoras de enfermedades, compiten con ellas, las atacan o se cruzan con ellas (Wittenberg \& Cock, 2001). 
La progresiva distribución espacial y temporal de L. fulica en la ciudad de Puerto Iguazú, se puede atribuir a la influencia antrópica, debido a la remoción y traslado de tierra para construcción de viviendas y calles, donde pueden encontrarse huevos de esta especie de molusco, como así también el uso de ejemplares adultos en la pesca artesanal. Este es uno de los factores determinantes de la dispersión no controlada del caracol. Algo similar sucede en Brasil, en donde la dispersión de L. fulica afectó a 24 estados del país vecino. Particularmente en el estado de Rio de Janeiro, se observó durante el período 2002-2006, un incremento en su distribución, logrando ocupar casi la totalidad de la extensión de este estado (Thiengo et al., 2007). En Colombia el escenario se repite, ya que el extremo sur del país linda con varios estados de Brasil donde se encuentra el caracol gigante africano. Su incremento en los últimos año afectó varias de sus cosechas (papayas, cítricos, mangos, calabaza, maíz), por lo cual trajo como consecuencias grandes pérdidas económicas (Linares et al., 2013).

Otra de las causas que suma a la expansión de L. fulica es su tasa de reproducción (5 o 6 veces al año) (Carvalho da Silva \& Pessoa Omena, 2014), que produce un incremento en el número de individuos de la población. Esta característica de L. fulica resulta en desventaja para los caracoles terrestre nativos (e.g. Megalobulimus spp.) cuya tasa de reproducción es más baja (2 veces al año) (Velásquez-Trujillo, 2012). La tasa reproductiva y la versatilidad para adaptarse a condiciones ambientales desfavorables que posee L. fulica, permitieron el aumento en su distribución espacial en los últimos años en la ciudad de Puerto Iguazú.

Estudios previos han reportado el comportamiento depredador de L. fulica, sugiriendo así un potencial impacto sobre otras especies (Srivastava, 1992). Así L. fulica ha sido indicado como depredador de babosas de la familia Veronicellidae, siendo capaces de consumir individuos de similar o mayor tamaño (Meyer et al., 2008). Esto podría explicar la disminución de los Veronicellidae en los sitios de muestreo durante el transcurso del presente trabajo. Estas babosas además son consideradas plaga para las huertas orgánicas, por lo cual se los ha controlado mediante la utilización de molusquicidas. Por otra parte estos moluscos (Veronicelidos) tienen hábitos nocturnos, momento del día en el que no es posible acceder a las viviendas para realizar muestreos. Esta situación podría determinar una subestimación del número poblacional. 
Distribución de tallas de los hospedadores en función del tiempo y de las variables ambientales.

Estudios previos han comprobado que las tallas de ejemplares de L. fulica encontradas más frecuentemente en la naturaleza son las tallas entre 3 a $9 \mathrm{~cm}$ de longitud (Albuquerque et al., 2007), esto explicaría el hecho de haber encontrado tan pocos ejemplares del intervalo de talla $4[9-12 \mathrm{~cm}$ ) durante los muestreos. Además $L$. fulica tiene un comportamiento gregario, y esta característica favorece el canibalismo entre individuos de una misma población, por lo cual disminuye la supervivencia y presencia de individuos de tallas más pequeñas (Fischer et al., 2005). Gutiérrez Gregoric et al (2013) en Corrientes Capital, registran que las tallas de 0-4 cm son la predominantes. Sin embargo las diferencias observadas con el presente trabajo y el anterior estudio, puede deberse a que Gregoric et al.(2013) basaron su trabajo en un único muestreo realizado en mayo en un solo sitio.

A partir del gráfico de distribución de tallas se puede observar un crecimiento poblacional de L. fulica desde el inicio hasta el final de esta investigación.

Teniendo en cuenta que $L$. fulica al eclosionar tiene un tamaño promedio de $5 \mathrm{~mm}$ (Fischer et al., 2010), y posee un crecimiento mensual de $15 \mathrm{~mm}$ promedio, se desprende que los individuos mencionados han eclosionado en el bimestre octubrenoviembre y que dicha cohorte proviene de ejemplares de caracoles que copularon en agosto-septiembre, luego de salir de la hibernación (junio-julio).

Contemplando que los individuos comienzan a activarse luego del período de hibernación con el aumento paulatino de las temperaturas, se desprende que la época más adecuada para combatir a $L$.fulica como plaga, es inmediatamente luego del periodo de hibernación (agosto-septiembre).

En relación a las babosas terrestres, se hallaron muy pocos ejemplares que superen los $10 \mathrm{~cm}$ de longitud. Esto puede explicarse en el hecho de tener un cuerpo desnudo y respiración a través del tegumento, por lo cual un mayor tamaño corporal afectaría la supervivencia por desecación en virtud de las altas temperaturas del ambiente. Además el ciclo de vida y desarrollo de las babosas está influenciado por las condiciones climáticas (temperatura y humedad) (Rodrigues Gomes, 2007). Esto explicaría el mayor número de ejemplares en el período octubre-noviembre, cuando las condiciones son favorables por mayor disponibilidad de recursos alimenticios y tasa de crecimiento, demostrado en el hallazgo de tallas más grandes. 
En el sentido contrario, las menores temperaturas registradas en el bimestre juniojulio, determinarían la ausencia de babosas, o el hallazgo de tallas más pequeñas.

\section{Distribución de nichos ecológicos de L. fulica}

El cambio climático que viene sufriendo el planeta en los últimos años está asociado a un aumento en los niveles de dióxido de carbono, y en consecuencia se observa un incremento en la temperatura global. La actividad humana ha contribuido de manera activa a que el efecto invernadero sea más notorio y progresivo en los últimos 20 años (i.e. quema de combustibles fósiles, deforestación). Durante los últimos 100 años la temperatura del aire superficial aumentó en promedio $0,6^{\circ}$ Celsius (Hassol, 2004).

Las proyecciones de los modelos de cambio climático muestran una gran variación atmosférica y de circulación oceánica, que trae como consecuencia la elevación del nivel del mar (los modelos predicen que el nivel del mar podría subir hasta $59 \mathrm{~cm}$ durante el siglo XXI) y una variación en el rango de precipitaciones (las temperaturas cálidas provocarán lluvias más intensas en algunas regiones). Estos cambios pueden tener impacto a escala individual, poblacional, de especie, comunidad, ecosistema y biomas, mostrando, en particular, que las especies pueden responder a los desafíos del cambio climático modificando su distribución, fenología, y fisiología. Por lo tanto es posible que las especies ya no se adapten al conjunto de condiciones ambientales de una región dada, quedando así fuera de su nicho climático, lo que trae como consecuencia que las relaciones interespecíficas (presa-depredador, hospedadorparásitos) se vean afectadas (Bellard et al., 2012).

A futuro se observa que el cambio climático modificará los ciclos de vida de las especies por lo cual la distribución espacial de las mismas se disminuirá, o expandirá a nuevas áreas impensadas, con alteraciones en el funcionamiento de los ecosistemas (Bellard et al., 2012).

Una especie exótica invasora debido a su plasticidad, puede adaptarse a nuevas condiciones ambientales, aumentado así su población y distribución geográfica, ya que son especies con gran capacidad de dispersión y colonización. Las especies invasoras son hoy, bajo cualquier punto de vista, uno de los principales factores de cambio global (Mack et al., 2000). 
El uso de datos de solo presencia de L. fulica para obtener los modelos constituyó una ventaja, ya que permitió visualizar en los mapas los "puntos calientes" (áreas de color rojo) de la distribución actual.

Las zonas más afectadas en el 2050 serian las que se localizan por debajo de la línea del Ecuador (áreas coloreadas en rojo y naranja). Por lo cual a futuro L. fulica cambiaría su distribución espacial hacia el este, oeste y sur de Sudamérica, quedando incluida casi la totalidad de Argentina.

Los resultados obtenidos en este trabajo son muy similares a los observados por Sarma et al., (2015) en la India, quienes predijeron una expansión de L fulica hacia regiones que en la actualidad son poco probables. Sin embargo teniendo en cuenta el cambio climático global y la biología de L. fulica, no es posible afirmar que su distribución pueda alcanzar los límites predichos a partir del análisis de distribución de nichos ecológicos elaborado. 


\section{Parte II: Los parásitos \\ RESULTADOS}

\subsection{Identificación de los helmintos}

Un total de 1.129 gasterópodos terrestres: Lissachatina fulica $(\mathrm{n}=995)$, Phyllocaulis variegatus $(\mathrm{n}=120)$ y Latipes erinaceus $(\mathrm{n}=14)$ fueron procesados a partir de diferentes técnicas:

\begin{tabular}{|l|c|c|}
\hline & Eviscerados & Digeridos \\
\hline Lissachatina fulica & 573 & 422 \\
\hline Phyllocaulis variegatus & 67 & 53 \\
\hline Latipes erinaceus & 5 & 9 \\
\hline
\end{tabular}

A partir del método de evisceración se halló una especie de Trematode, Brachylaima sp. en las babosas Veronicellidae, y una especie de nematode, Strongyluris sp., en L. fulica y Veronicelidos.

La única especie hallada a partir del método de digestión artificial fue Aelurostrongylus abstrusus en L. fulica.

Nunca fueron halladas dos especies de nematodes en un mismo ejemplar. 
Clase Trematoda Rudolphi, 1808

Subclase Digenea Carus, 1863

Familia Brachylaimidae Joyeaux \& Foley, 1930

Los miembros de esta familia se caracterizan por poseer una ventosa oral bien desarrollada, situada en la parte anterior del cuerpo. Presenta una faringe muscular notoria, ciegos intestinales largos que terminan cerca de la extremidad posterior. En sus ciclos de vida se incluyen dos hospedadores intermediarios que son moluscos terrestres.

Brachylaima Dujardin, 1843

\section{Brachylaima sp. (metacercaria)}

Descripción: Cuerpo elongado en forma de lengüeta, 1670 a $2280 \mu \mathrm{m}(1976 \mu \mathrm{m})$ por 480 a $750 \mu \mathrm{m}(639 \mu \mathrm{m})$. La ventosa oral, 250 a $350 \mu \mathrm{m}(291 \mu \mathrm{m})$ por 230 a $350 \mu \mathrm{m}$ $(279 \mu \mathrm{m})$. Presenta una faringe muscular bien desarrollada, 140 a $180 \mu \mathrm{m}(155 \mu \mathrm{m})$ por $140-170 \mu \mathrm{m}(150 \mu \mathrm{m})$, los ciegos intestinales son sinuosos y se extienden casi hasta la parte posterior del cuerpo. La ventosa ventral está ubicada en el primer tercio del cuerpo, 250-350 $\mu \mathrm{m}(291 \mu \mathrm{m})$ por 230-350 $\mu \mathrm{m}(279 \mu \mathrm{m})$. Las gónadas están localizadas en la parte posterior del cuerpo muy cercana a la extremidad posterior: el testículo anterior, 65 a $100 \mu \mathrm{m}(76 \mu \mathrm{m})$ por 50 a $84 \mu \mathrm{m}(66 \mu \mathrm{m})$; el testículo posterior, 66 a $100 \mu \mathrm{m}(83 \mu \mathrm{m})$ por 50 a $93 \mu \mathrm{m}(67 \mu \mathrm{m})$, el ovario es intertesticular 55 a $92 \mu \mathrm{m}$ (72) por 50 a $80 \mu \mathrm{m}(64 \mu \mathrm{m})$. El poro genital está localizado en el margen anterior del testículo anterior. Las glándulas vitelinas se extienden desde el margen anterior hasta la ventosa posterior. El útero se extiende desde la bifurcación del intestino hasta el margen anterior de la ventosa ventral (Figura 32) (CHMLP He 6914).

Material estudiado: 10 ejemplares teñidos y montados in toto

\section{Hospedadores: Phyllocaulis variegatus}

Localización: Poro genital femenino

Ciclo de vida: Esta familia tiene ciclos terrestres heteroxenos que incluyen a 2 hospedadores intermediaros (HI) que pueden ser caracoles o babosa terrestres, y una variedad de hospedadores definitivos (HD) tales como aves, mamíferos, reptiles y anfibios. El HD libera sus huevos a través de las heces, el $1^{\circ} \mathrm{HI}$ consume los huevos 
que contienen al miracidio. En el $1^{\circ} \mathrm{HI}$ el miracidio da lugar al esporocisto en la glándula digestiva. Los esporocistos generan cercarias, que son emitidas al exterior a través de la baba del $1^{\circ} \mathrm{HI}$. Las cercarias penetran en el $2^{\circ} \mathrm{HI}$ y maduran a metacercarias (las cuales no se enquistan) en los metanefridios, donde usualmente se encuentran. Este $2^{\circ} \mathrm{HI}$ es consumido por un $\mathrm{HD}$, en el cual la metacercaria madura dando lugar al adulto (Yamaguti, 1975). Varios casos de infestaciones humanas han tenido síntomas gastrointestinales. Los principales son diarrea mucosa, acuosa, dolor abdominal, anorexia y/o pérdida de peso. La fuente probable de infestación es la ingestión de molusco crudos o el consumo de hortalizas cultivadas contaminadas con secreción de caracoles infectados (Butcher \& Groove, 2001).

Comentarios: Brachylaima es un género cosmopolita que comprende hasta el momento 72 especies. Fue descripto originalmente como Brachylaimus Dujardin, 1843, sin embargo el nombre fue latinizado por el mismo autor, pasando así a Brachylaima.

En este trabajo las metacercarias fueron halladas en el poro genital femenino, en contraste con estudios previos que citan a este género en el saco del pericardio (Thiengo \& Amato, 1995) o en los metanefridios (Barger \& Hnida, 2008).

Existen varios registros previos de adultos de Brachylaima parasitando aves y mamíferos en Argentina (Martínez, 1986; Lunaschi \& Drago, 2012). Brachylaima migrans Dujardin, 1845 fue encontrado en la zarigüeya Didelphis albiventris (Mammalia: Marsupialia) en Corrientes, Chaco y Formosa. Este mamífero tiene hábitos principalmente frugívoro-omnívoro, aunque los invertebrados (caracoles, babosas, gusanos y artrópodos) constituyen una parte importante de su dieta, por lo cual se ha especulado que los caracoles están involucrados como hospedadores intermediarios en el ciclo de vida de estos parásitos (Santa Cruz, 2006). Particularmente en la provincia de Misiones, se observaron trematodos adultos del género Brachylaima en el intestino de Didelphis aurita (Mammalia: Marsupialia) (Navone, com. pers.).

Este hallazgo constituye el primer reporte de metacercarias Brachylaima sp. en Argentina (Valente et al., 2016a). 


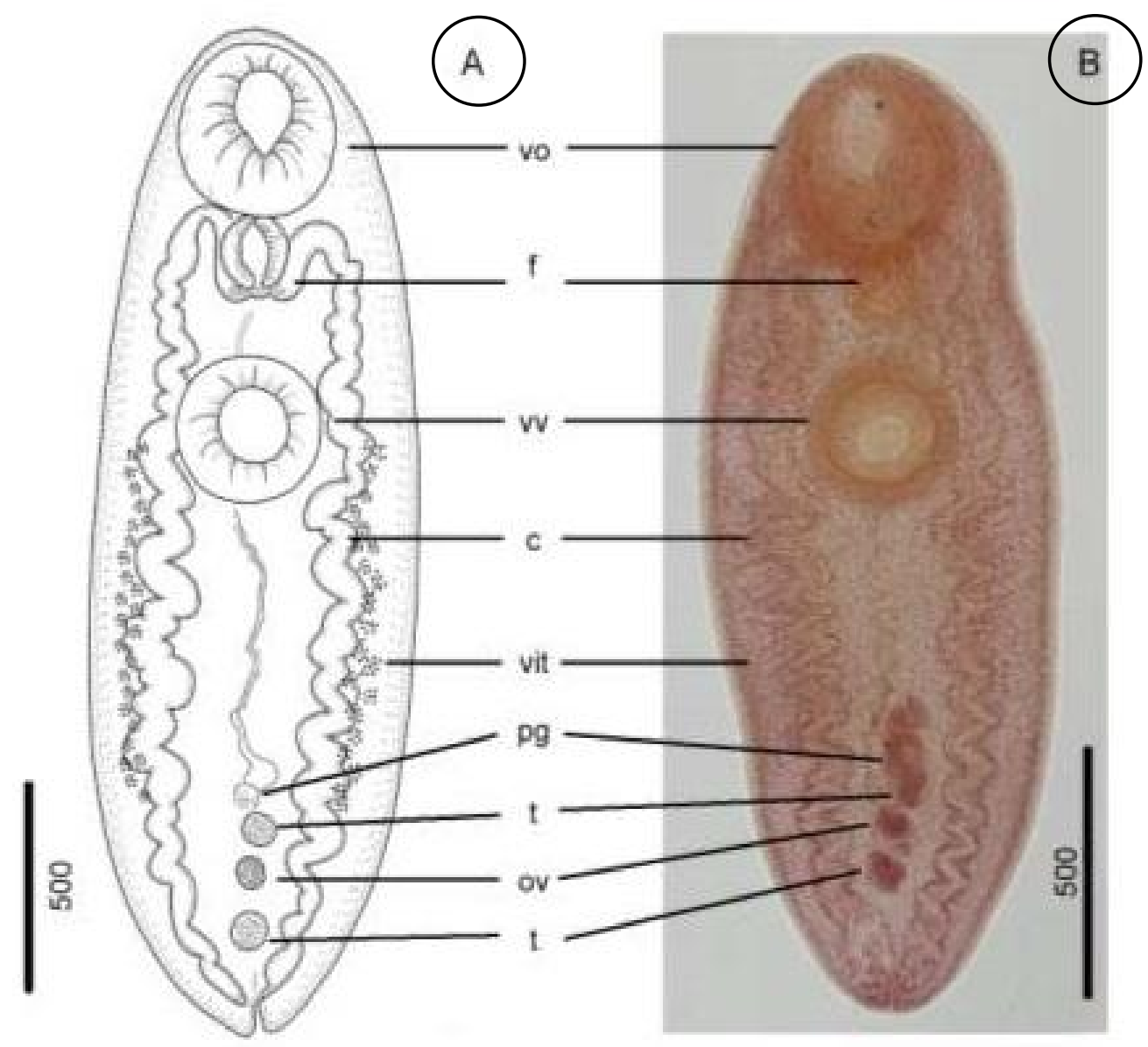

Figura 32. A) Brachylaima sp. (metacercaria) ( $\mu \mathrm{m})$. B) Brachylaima sp. (metacercaria) (MO). Referencias: $\mathbf{v o}=$ ventosa oral, $\mathbf{f}=$ faringe, $\mathbf{v v}=$ ventosa ventral, $\mathbf{c}=$ ciegos intestinales, $\mathbf{v i t}=$ glándulas vitelinas, $\mathbf{p g}=$ primordio genital, $\mathbf{t}=$ testículos, $\mathbf{o v}=$ ovario. 
Clase Nematoda Rudoplhi, 1808

Subclase Secernentea

\section{Orden Ascaridida}

Familia Heterakidae Railliet \& Henry, 1912

Los miembros de esta familia están caracterizados por presentar un esófago muscular corto que termina en un bulbo posterior. La boca está rodeada de labios redondeados, que no están conectados.

Strongyluris Müieller, 1894

\section{Strongyluris sp. (L3)}

Descripción: Cutícula blanca estriada transversalmente. El cuerpo mide entre $2.090 \mu \mathrm{m}$ y $4.590 \mu \mathrm{m}(2.770 \mu \mathrm{m})$ de largo por $280 \mu \mathrm{m}$ a $330 \mu \mathrm{m}(305 \mu \mathrm{m})$ de ancho. Boca rodeada por tres labios con estructuras sensoriales como papilas y ánfidos. Esófago muscular bien desarrollado, entre $300 \mu \mathrm{m}$ y $650 \mu \mathrm{m}(525 \mu \mathrm{m})$ que termina en un bulbo esofágico. En el extremo posterior se observa la abertura del sistema digestivo que varía entre $80 \mu \mathrm{m}$ a $450 \mu \mathrm{m}(270 \mu \mathrm{m})$ (Figura 33) (CMLP He 7020).

Material estudiado: 10 ejemplares

Hospedador: Lissachatina fulica y Phyllocaulis variegatus

Localización: Se encontraron especímenes en forma enquistada en ambas especies hospedadoras; en el caso de L. fulica se encontraron en la cavidad del manto (pulmón) y en $P$. variegatus se hallaron a lo largo del cordón nervioso pedal.

Ciclo de vida: La bibliografía cita a este género con ciclo de vida directo. El HD libera el huevo conteniendo a la L1, dentro del mismo se producen dos mudas, llegando al estadio de L3 que es el estado infectivo. Esta L3 permanece dentro del huevo hasta ser consumido por el HD (Anderson et al., 2010). Sin embargo, se ha descrito el ciclo de Strongyluris oscari Travassos, 1923 como un ciclo de vida indirecto, en donde el huevo conteniendo a la larva infectiva es consumido por un artrópodo o por un molusco, los cuales actúan como hospedadores paraténicos o de transporte (Barreto-Lima \& Alves dos Anjos, 2014). 
Comentarios: El género Strongyluris incluye un total de 32 especies que son parásitas del intestino de reptiles y anfibios. En la región Neotropical se registraron cinco especies: S. acaudata Caballero, 1941; S. oscari Travassos, 1923; S. panamaensis Bursey, Goldberg \& Telford, 2003; S. similis Caballero, 1934 y S. amazonicus dos
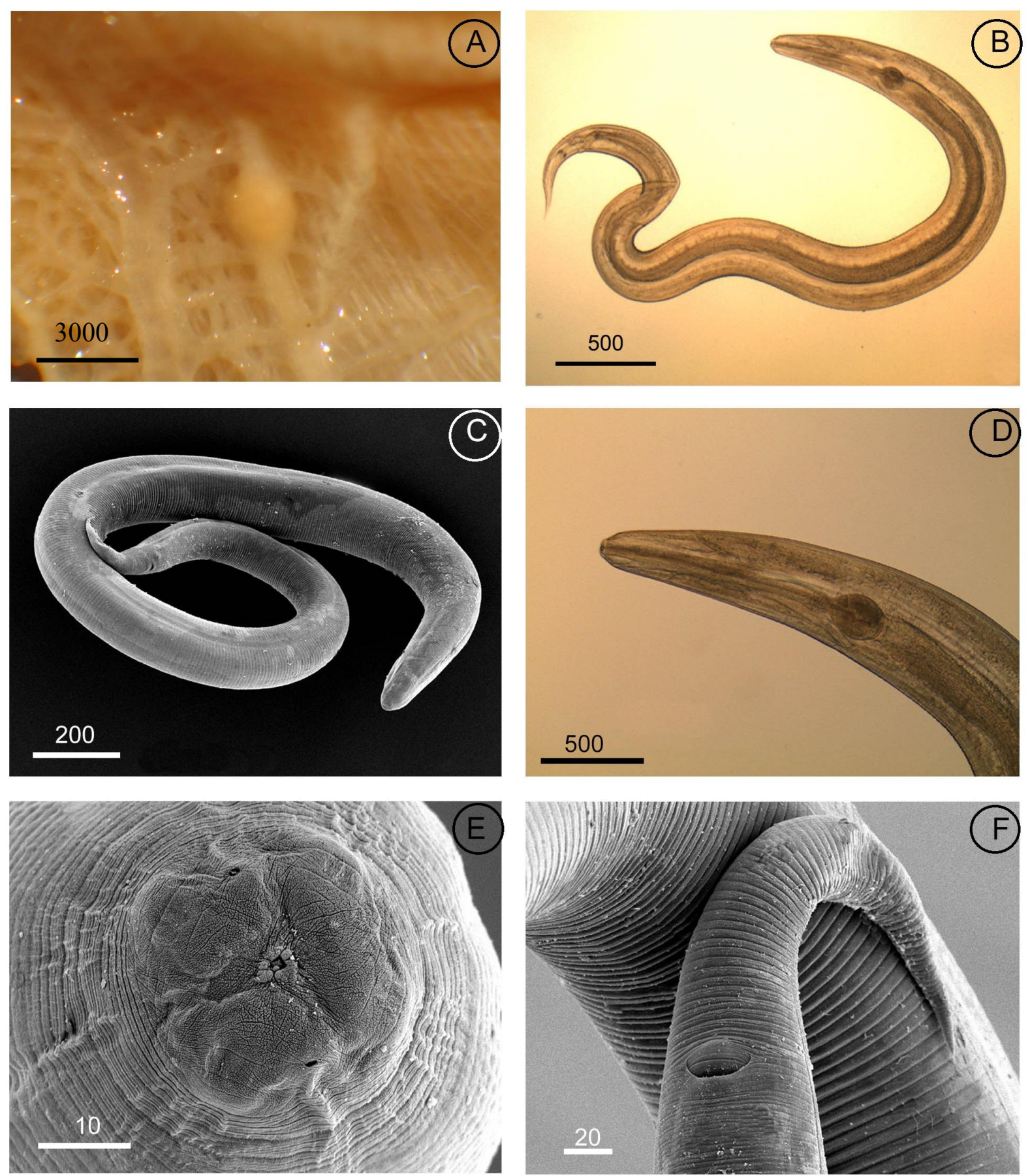

Figura 33. Strongyluris sp $(\mu \mathrm{m})$. A) Nódulo en cavidad paleal, B) Juvenil (L3) desenquistado (MO), C) Juvenil (L3) (MEB), D) Extremo anterior (MO), E) Extremo anterior, vista apical (MEB), F) Extremo posterior (MEB). 


\section{Orden Strongylida}

\section{Superfamilia Metastrongyloidea}

Familia Angiostrongylidae (Boehm \& Grauer, 1934)

Los miembros de esta familia son denominados comúnmente como "gusanos de pulmón", ya que muchas especies se encuentran dentro de los pulmones o en la arteria pulmonar de los hospedadores definitivos (e.g. marsupiales, insectívoros, carnívoros y roedores). Se caracterizan por poseer los machos una típica bursa y las hembras una vulva posterior. Incluyen en su ciclo de vida a gasterópodos como hospedadores intermediarios.

\section{Aelurostrongylus abstrusus (L3) (Railliet, 1898)}

Descripción: Una o dos vainas cuticulares. En la porción bucal presenta un par de estiletes, seguidos por un largo esófago cilíndrico y muscular que varía entre $190 \mu \mathrm{m}$ y $215 \mu \mathrm{m}(201 \mu \mathrm{m})$. El cuerpo mide entre $500 \mu \mathrm{m}$ y $635 \mu \mathrm{m}(562 \mu \mathrm{m})$ de largo por $23 \mu \mathrm{m}$ y $38 \mu \mathrm{m}(30 \mu \mathrm{m})$ de ancho. Se observa un incipiente poro excretor, distante entre $90 \mu \mathrm{m}$ y $99 \mu \mathrm{m}(95 \mu \mathrm{m}) \mathrm{d}$ el extremo anterior. En el extremo posterior se observa la abertura del sistema digestivo, distante entre $20 \mu \mathrm{m}$ y $35 \mu \mathrm{m}(29 \mu \mathrm{m})$ del extremo posterior, cercano a ello se distinguen los primordios genitales localizados a $150 \mu \mathrm{m}$ a $180 \mu \mathrm{m}$ $(170 \mu \mathrm{m})$ del cuerpo. Esta especie es reconocida ya que su extremo posterior termina en forma de "botón redondeado" (Figura 34) (CMLP He7253).

Material estudiado: 10 ejemplares

\section{Hospedador: Lissachatina fulica}

Localización: secreción mucosa

Ciclo de vida: Esta especie posee un ciclo de vida indirecto que incluye a dos hospedadores. Los adultos habitan las vías respiratorias (e.g. alveolos pulmonares) de sus HD, félidos silvestres o domésticos. La L1 eclosiona en el pulmón y sube hasta la tráquea donde es deglutida para ser liberada con las heces. La L1 es consumida por un caracol que actúa como HI, allí sufre dos mudas hasta llegar a L3. El ciclo puede incluir hospedadores paraténicos o de transporte, tales como sapos, ranas o lagartijas que pueden alojar a la L3. El HD se infecta cuando ingiere la L3 a través del consumo del HI o del hospedador paraténico. 
Comentarios: Esta especie tiene una amplia distribución en países de Europa (e.g. Italia, España, Francia). En Argentina se lo reportó en gatos domésticos de Buenos Aires, Corrientes y Santa Fe, causando patologías respiratorias tales como estornudos, secreción ocular-nasal y pérdida progresiva de peso. En algunos casos puede causar la muerte. Recientemente fue detectada la presencia de A. abstrusus en Rumina decollata (Linnaeus, 1758), un caracol exótico presente en Argentina (Cardillo et al., 2014).

Este hallazgo representa el primer registro de infestación natural de Aelurostrongylus abstrusus en L. fulica en Argentina (Valente et al., 2017).
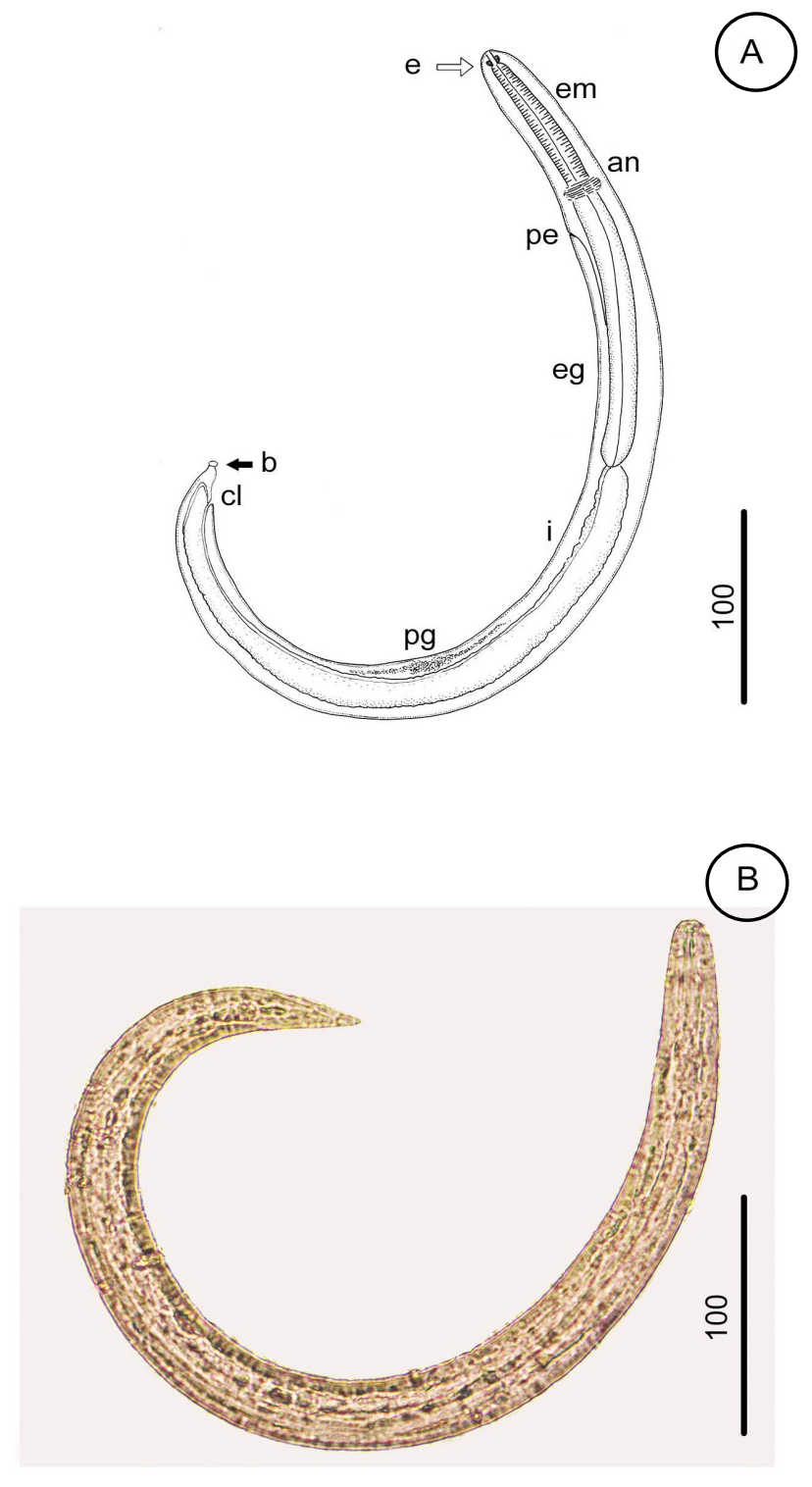

Figura 34. A) Aelurostrongylus abstrusus (L3) ( $\mu \mathrm{m})$. B) Aelurostrongylus abstrusus (L3) (MO). Referencias: $\mathbf{e}=$ estiletes, $\mathbf{e m}=$ esófago muscular, $\mathbf{a n}=$ anillo nervioso, $\mathbf{p e}=$ poro excretor, $\mathbf{e g}=$ estómago glandular, $\mathbf{i}=$ intestino, $\mathbf{p g}=$ primordio genital, $\mathbf{c l}=$ cloaca, b= botón. 


\section{Comentarios acerca de los resultados obtenidos a partir de las metodologías aplicadas}

Los helmintos hallados en las especies hospedadoras fueron diferentes según la metodología aplicada (método de evisceración vs. método de digestión artificial). Esto fue particularmente observado para L. fulica, lo cual sería el reflejo de una eficacia diferencial de cada una de las metodologías, o algún tipo de error de procedimiento.

Con respecto al método de evisceración que se realiza bajo lupa, si bien este protocolo de procesamiento es el utilizado en los laboratorios de parasitología (Pritchard \& Kruse, 1982) y ha sido utilizado en investigaciones similares (Franco-Acuña et al., 2009), es probable que no se hayan podido visualizar larvas de tan pequeño tamaño como son las larvas de A. abstrusus (500 a $700 \mu \mathrm{m}$ ), en contraparte con la observación de larvas de mayor tamaño como son las larvas de Strongyluris sp. $(3.000 \mu \mathrm{m})$.

En la metodología de digestión se procede a filtrar la solución digestiva a través de un embudo que contiene un colador con una malla de $1 \mathrm{~mm}$ x $1 \mathrm{~mm}$, por encima de ese embudo se coloca una gasa (que filtra el paso de los restos de masa visceral que no fueron revisados). Es probable que el tamaño del poro utilizado en esta investigación haya favorecido el paso de las larvas más pequeñas, (A. abstrusus) y haya impedido el camino de las larvas más grandes (Strongyluris sp.). Este protocolo es el utilizado en el Laboratorio de Referencia en Malacología del Instituto Oswaldo Cruz de Rio de Janeiro (Directora del Laboratorio de Referencia en Malacología. Dra. Silvana Thiengo) quienes si han encontrado ambos tipos de larvas a partir de este procedimiento, por lo cual no puede atribuirse a una cuestión de competencia.

Si bien Thiengo y colaboradores (com. pers.) han obtenido larvas de ambas especies a partir de la utilización de este método, el tamaño de los moluscos analizados por ellos es en general menor (aproximadamente $6 \mathrm{~cm}$ ), mientras que los moluscos analizados en el presente trabajo tienen diferente rango de talla, sobrepasando la mayoría de ellos los $6 \mathrm{~cm}$, alcanzando a medir $10 \mathrm{~cm}$. Teniendo en cuenta que el tamaño del hospedador puede influenciar sobre el tamaño de los parásitos (Poulin, 2007), es probable que las larvas de Strongyluris sp. parasitando los presentes hospedadores alcancen tallas que no le permitan atravesar la malla. 


\subsection{Parámetros poblacionales y su relación con las tallas de los hospedadores}

\section{Lissachatina fulica}

Para el caso de Strongyluris sp. los parámetros poblacionales fueron: $\mathrm{P}=7,15 \%$, $\mathrm{AM}=0,53$ y $\mathrm{IM}=7,44$. En la tabla 5 y figura 35 se muestran la prevalencia, intensidad media y abundancia media de Strongyluris sp. en relación a las intervalos de tallas de $L$. fulica. Se observa una correlación positiva y significativa entre la prevalencia de infección, intensidad media y el tamaño del hospedador $(\boldsymbol{r s}=\mathbf{0 , 9})$. La intensidad de infección varió de 0-14. La mayor infección se observó en la talla de [9-12) cm.

Los parámetros poblacionales para A. abstrusus fueron: $\mathrm{P}=4,74 \%, \mathrm{AM}=0,48 \mathrm{e}$ $\mathrm{IM}=10,3$. La tabla $6 \mathrm{y}$ figura 36 muestran la prevalencia, intensidad media y abundancia media de A. abstrusus en relación a las intervalos de tallas de L. fulica. Se observó una correlación positiva y significativa entre la prevalencia de infección, intensidad media y el tamaño del hospedador $(\boldsymbol{r} \boldsymbol{s}=\mathbf{0 , 5 5})$. La intensidad de infección varió de 2-27. La mayor infección se encontró en el intervalo de talla [6-9) cm. 
Tabla 5. Parámetros ecológicos de Strongyluris sp. en Lissachatina fulica por intervalo de talla y bimestre.

\begin{tabular}{|c|c|c|c|c|c|c|c|c|}
\hline $\begin{array}{l}\text { Intervalos de } \\
\text { tamaño }\end{array}$ & Oct-Nov 2013 & Feb-Mar 2014 & Jun-Jul 2014 & Oct-Nov 2014 & Feb-Mar 2015 & Jun-Jul 2015 & Oct-Nov 2015 & SubTotal \\
\hline \multirow{4}{*}{$\begin{array}{c}1 \\
{[0-3 \mathrm{~cm})}\end{array}$} & $\mathbf{n}=11$ & $\mathbf{n}=4$ & $\mathbf{n}=17$ & $\mathbf{n}=11$ & $\mathbf{n}=9$ & $\mathbf{n}=22$ & $\mathbf{n}=4$ & \multirow{4}{*}{$\begin{array}{c}\mathbf{N}=78 \\
\mathbf{P}=0 \\
\mathbf{I M}=0 \\
\mathbf{A M}=0\end{array}$} \\
\hline & $\mathbf{P}=0$ & $\mathbf{P}=0$ & $\mathbf{P}=0$ & $\mathbf{P}=0$ & $\mathbf{P}=0$ & $\mathbf{P}=0$ & $\mathbf{P}=0$ & \\
\hline & $\mathbf{I M}=0$ & $\mathbf{I M}=0$ & $\mathbf{I} \mathbf{M}=0$ & $\mathbf{I} \mathbf{M}=0$ & $\mathbf{I M}=0$ & $\mathbf{I M}=0$ & $\mathbf{I M}=0$ & \\
\hline & $\mathbf{A M}=0$ & $\mathbf{A M}=0$ & $\mathbf{A M}=0$ & $\mathbf{A M}=0$ & $\mathbf{A M}=0$ & $\mathbf{A M}=0$ & $\mathbf{A M}=0$ & \\
\hline \multirow{4}{*}{$\begin{array}{c}2 \\
{[3-6 \mathrm{~cm})}\end{array}$} & $\mathbf{n}=37$ & $\mathbf{n}=8$ & $\mathbf{n}=15$ & $\mathbf{n}=48$ & $\mathbf{n}=32$ & $\mathbf{n}=39$ & $\mathbf{n}=107$ & \multirow{4}{*}{$\begin{aligned} \mathbf{N} & =286 \\
\mathbf{P} & =3,84 \\
\mathbf{I M} & =3,9 \\
\mathbf{A M} & =0,15\end{aligned}$} \\
\hline & $\mathbf{P}=5,4$ & $\mathbf{P}=0$ & $\mathbf{P}=0$ & $\mathbf{P}=0$ & $\mathbf{P}=2$ & $\mathbf{P}=0$ & $\mathbf{P}=6,5$ & \\
\hline & $\mathbf{I M}=2,5$ & $\mathbf{I M}=0$ & $\mathbf{I} \mathbf{M}=0$ & $\mathbf{I M}=0$ & $\mathbf{I M}=2$ & $\mathbf{I M}=0$ & $\mathbf{I M}=4,8$ & \\
\hline & $\mathbf{A M}=0,1$ & $\mathbf{A M}=0$ & $\mathbf{A M}=0$ & $\mathbf{A M}=0$ & $\mathbf{A M}=0,1$ & $\mathbf{A M}=0$ & $\mathbf{A M}=0,3$ & \\
\hline \multirow{4}{*}{$\begin{array}{c}3 \\
{[6-9 \mathrm{~cm})}\end{array}$} & $\mathbf{n}=18$ & $\mathbf{n}=17$ & $\mathbf{n}=22$ & $\mathbf{n}=31$ & $n=52$ & $\mathbf{n}=31$ & $\mathbf{n}=28$ & \multirow{4}{*}{$\begin{array}{c}\mathbf{N}=199 \\
\mathbf{P}=13 \\
\mathbf{I M}=11,84 \\
\mathbf{A M}=1,54\end{array}$} \\
\hline & $\mathbf{P}=11,7$ & $\mathbf{P}=11,76$ & $\mathbf{P}=0$ & $\mathbf{P}=22,5$ & $\mathbf{P}=9,6$ & $\mathbf{P}=9,67$ & $\mathbf{P}=25$ & \\
\hline & $\mathbf{I M}=16,5$ & $\mathbf{I M}=16,5$ & $\mathbf{I M}=0$ & $\mathbf{I M}=5,4$ & $\mathbf{I M}=12$ & $\mathbf{I M}=23,6$ & $\mathbf{I M}=12,8$ & \\
\hline & $\mathbf{A M}=1,9$ & $\mathbf{A M}=1,94$ & $\mathbf{A M}=0$ & $\mathbf{A M}=1,2$ & $\mathbf{A M}=1,1$ & $\mathbf{A M}=2,3$ & $\mathbf{A M}=3,2$ & \\
\hline \multirow{4}{*}{$\begin{array}{c}4 \\
{[9-12 \mathrm{~cm})}\end{array}$} & $\mathbf{n}=1$ & $\mathbf{n}=4$ & $\mathbf{n}=1$ & $\mathbf{n}=0$ & $\mathbf{n}=2$ & $\mathbf{n}=1$ & $\mathbf{n}=1$ & \multirow{4}{*}{$\begin{array}{c}\mathbf{N}=10 \\
\mathbf{P}=30 \\
\mathbf{I M}=13,66 \\
\mathbf{A M}=4,1\end{array}$} \\
\hline & $\mathbf{P}=0$ & $\mathbf{P}=0$ & $\mathbf{P}=0$ & $\mathbf{P}=0$ & $\mathbf{P}=50$ & $\mathbf{P}=100$ & $\mathbf{P}=100$ & \\
\hline & $\mathbf{I M}=0$ & $\mathbf{I M}=0$ & $\mathbf{I M}=0$ & $\mathbf{I M}=0$ & $\mathrm{IM}=26$ & $\mathrm{IM}=5$ & $\mathbf{I M}=10$ & \\
\hline & $\mathbf{A M}=0$ & $\mathbf{A M}=0$ & $\mathbf{A M}=0$ & $\mathbf{A M}=0$ & $\mathbf{A M}=13$ & $\mathrm{AM}=5$ & $\mathbf{A M}=10$ & \\
\hline Subtotal & $\begin{array}{c}\mathbf{N}=67 \\
\mathbf{P}=5,97 \\
\mathbf{I M}=5,25 \\
\mathbf{A M}=0,31\end{array}$ & $\begin{array}{c}\mathbf{N}=33 \\
\mathbf{P}=6,06 \\
\mathbf{I M}=16,5 \\
\mathbf{A M}=1\end{array}$ & $\begin{array}{c}\mathbf{N}=55 \\
\mathbf{P}=0 \\
\mathbf{I M}=0 \\
\mathbf{A M}=0\end{array}$ & $\begin{array}{c}\mathbf{N}=90 \\
\mathbf{P}=7,77 \\
\mathbf{I M}=5,42 \\
\mathbf{A M}=0,42\end{array}$ & $\begin{array}{c}\mathbf{N}=95 \\
\mathbf{P}=8,42 \\
\mathbf{I M}=11,25 \\
\mathbf{A M}=0,94\end{array}$ & $\begin{array}{c}\mathbf{N}=93 \\
\mathbf{P}=4,3 \\
\mathbf{I M}=19 \\
\mathbf{A M}=0,81\end{array}$ & $\begin{array}{c}\mathbf{N}=140 \\
\mathbf{P}=10,71 \\
\mathbf{I M}=8,93 \\
\mathbf{A M}=0,95\end{array}$ & $\begin{array}{c}\text { TOTAL } \\
\mathbf{5 7 3}\end{array}$ \\
\hline
\end{tabular}

$\mathbf{P}=$ Prevalencia $; \mathbf{I M}=$ Intensidad media $\mathbf{A M}=$ Abundancia media. 
Tabla 6. Parámetros ecológicos de Aelurostrongylus abstrusus en Lissachatina fulica por intervalo de talla y bimestre.

\begin{tabular}{|c|c|c|c|c|c|c|}
\hline $\begin{array}{c}\text { Intervalos de } \\
\text { tamaño }\end{array}$ & Feb-Mar 2014 & Oct-Nov 2014 & Feb-Mar 2015 & Jun-Jul 2015 & Oct-Nov 2015 & SubTotal \\
\hline \multirow{4}{*}{$\begin{array}{c}1 \\
{[0-3 \mathrm{~cm})}\end{array}$} & $\mathbf{n}=4$ & $\mathbf{n}=28$ & $\mathbf{n}=10$ & $n=43$ & $\mathbf{n}=2$ & \multirow{4}{*}{$\begin{array}{c}\mathbf{N}=87 \\
\mathbf{P}=2,3 \\
\mathbf{I M}=2 \\
\mathbf{A M}=0,04\end{array}$} \\
\hline & $\mathbf{P}=0$ & $\mathbf{P}=7,14$ & $\mathbf{P}=0$ & $\mathbf{P}=0$ & $\mathbf{P}=0$ & \\
\hline & $\mathbf{I} \mathbf{M}=0$ & $\mathbf{I M}=2$ & $\mathbf{I} \mathbf{M}=0$ & $\mathbf{I M}=0$ & $\mathbf{I M}=0$ & \\
\hline & $\mathbf{A M}=0$ & $\mathbf{A M}=0,1$ & $\mathbf{A M}=0$ & $\mathbf{A M}=0$ & $\mathbf{A M}=0$ & \\
\hline \multirow{4}{*}{$\begin{array}{c}2 \\
{[3-6 \mathrm{~cm})}\end{array}$} & $\mathbf{n}=2$ & $\mathbf{n}=42$ & $\mathbf{n}=21$ & $\mathbf{n}=29$ & $\mathbf{n}=110$ & \multirow{4}{*}{$\begin{array}{c}\mathbf{N}=204 \\
\mathbf{P}=2,45 \\
\mathbf{I M}=2,6 \\
\mathbf{A M}=0,06\end{array}$} \\
\hline & $\mathbf{P}=0$ & $\mathbf{P}=2,3$ & $\mathbf{P}=0$ & $\mathbf{P}=0$ & $\mathbf{P}=3,63$ & \\
\hline & $\mathbf{I M}=0$ & $\mathbf{I M}=6$ & $\mathbf{I M}=0$ & $\mathbf{I M}=0$ & $\mathbf{I M}=1,75$ & \\
\hline & $\mathbf{A M}=0$ & $\mathbf{A M}=0,1$ & $\mathbf{A M}=0$ & $\mathbf{A M}=0$ & $\mathbf{A M}=0,06$ & \\
\hline \multirow{4}{*}{$\begin{array}{c}3 \\
{[6-9 \mathrm{~cm})}\end{array}$} & $\mathbf{n}=10$ & $\mathbf{n}=20$ & $\mathbf{n}=49$ & $\mathbf{n}=18$ & $\mathbf{n}=23$ & \multirow{4}{*}{$\begin{array}{c}\mathbf{N}=120 \\
\mathbf{P}=9,16 \\
\mathbf{I M}=26,8 \\
\mathbf{A M}=17\end{array}$} \\
\hline & $\mathbf{P}=0$ & $\mathbf{P}=30$ & $\mathbf{P}=4$ & $\mathbf{P}=0$ & $\mathbf{P}=13$ & \\
\hline & $\mathbf{I M}=0$ & $\mathbf{I M}=15$ & $\mathbf{I M}=45$ & $\mathbf{I} \mathbf{M}=0$ & $\mathbf{I M}=2$ & \\
\hline & $\mathbf{A M}=0$ & $\mathbf{A M}=4,6$ & $\mathbf{A M}=1,83$ & $\mathbf{A M}=0$ & $\mathbf{A M}=0,26$ & \\
\hline \multirow{4}{*}{$\begin{array}{c}4 \\
{[9-12 \mathrm{~cm})}\end{array}$} & $\mathbf{n}=4$ & $\mathbf{n}=2$ & $\mathbf{n}=2$ & $\mathbf{n}=0$ & $\mathbf{n}=3$ & \multirow{4}{*}{$\begin{array}{c}\mathbf{N}=11 \\
\mathbf{P}=9 \\
\mathbf{I M}=1 \\
\mathbf{A M}=0,09\end{array}$} \\
\hline & $\mathbf{P}=0$ & $\mathbf{P}=0$ & $\mathbf{P}=0$ & $\mathbf{P}=0$ & $\mathbf{P}=33,3$ & \\
\hline & $\mathbf{I M}=0$ & $\mathbf{I} \mathbf{M}=0$ & $\mathbf{I} \mathbf{M}=0$ & $\mathbf{I} \mathbf{M}=0$ & $\mathbf{I M}=1$ & \\
\hline & $\mathbf{A M}=0$ & $\mathbf{A M}=0$ & $\mathbf{A M}=0$ & $\mathbf{A M}=0$ & $\mathbf{A} \mathbf{M}=0,33$ & \\
\hline Subtotal & $\begin{array}{c}\mathbf{N}=20 \\
\mathbf{P}=0 \\
\mathbf{I M}=0 \\
\mathbf{A M}=0\end{array}$ & $\begin{array}{c}\mathbf{N}=92 \\
\mathbf{P}=9,78 \\
\mathbf{I M}=11,3 \\
\mathbf{A M}=1,10\end{array}$ & $\begin{array}{c}\mathbf{N}=82 \\
\mathbf{P}=2,43 \\
\mathbf{I M}=45 \\
\mathbf{A M}=1,09\end{array}$ & $\begin{array}{c}\mathbf{N}=90 \\
\mathbf{P}=0 \\
\mathbf{I M}=0 \\
\mathbf{A M}=0\end{array}$ & $\begin{array}{c}\mathbf{N}=138 \\
\mathbf{P}=5,79 \\
\mathbf{I M}=1,75 \\
\mathbf{A M}=0,10\end{array}$ & $\begin{array}{c}\text { TOTAL } \\
422\end{array}$ \\
\hline
\end{tabular}

$\mathbf{P}=$ Prevalencia $\mathbf{I M}=$ Intensidad media; $\mathbf{A M}=$ Abundancia media. 


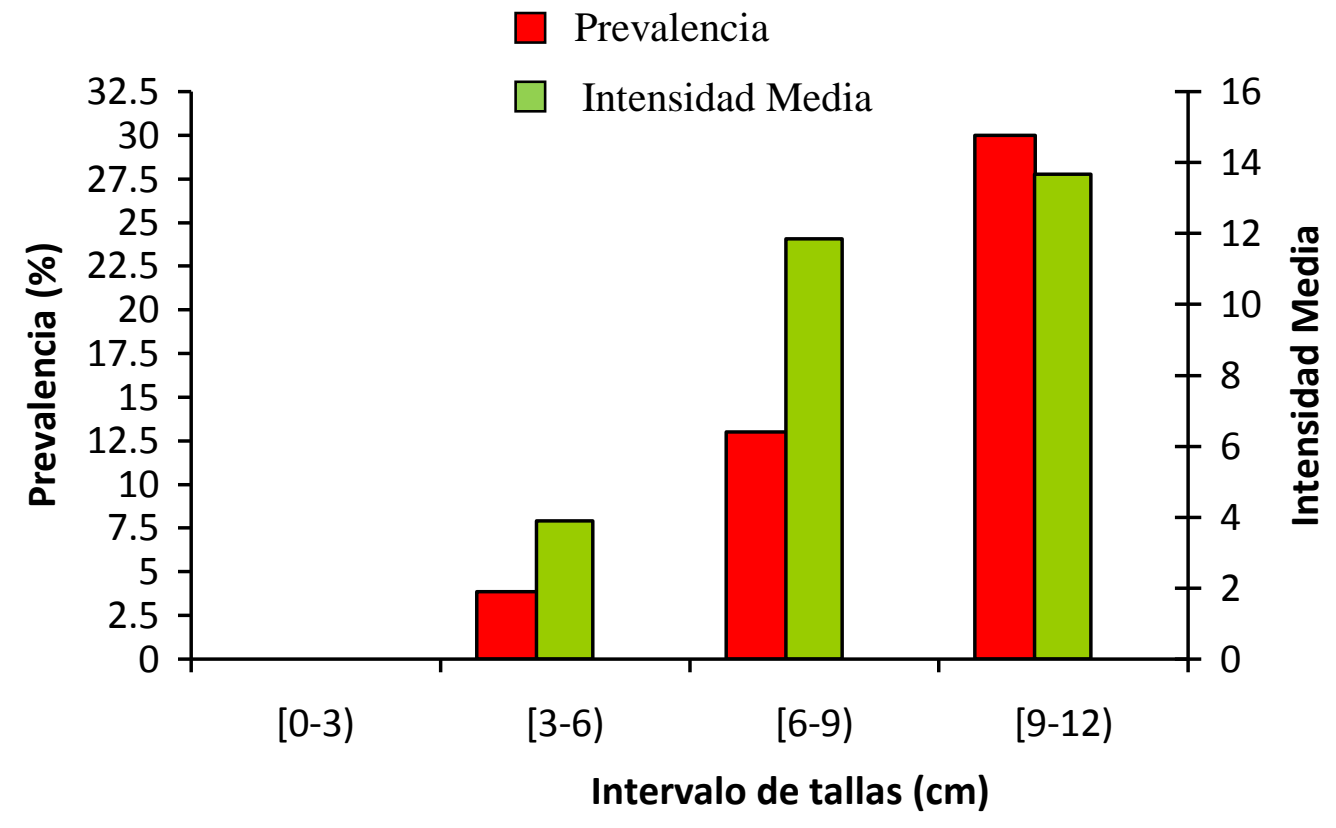

Figura 35. Intensidad media $v s$ prevalencia de Strongyluris sp. en relación a los intervalos de talla de Lissachatina fulica.

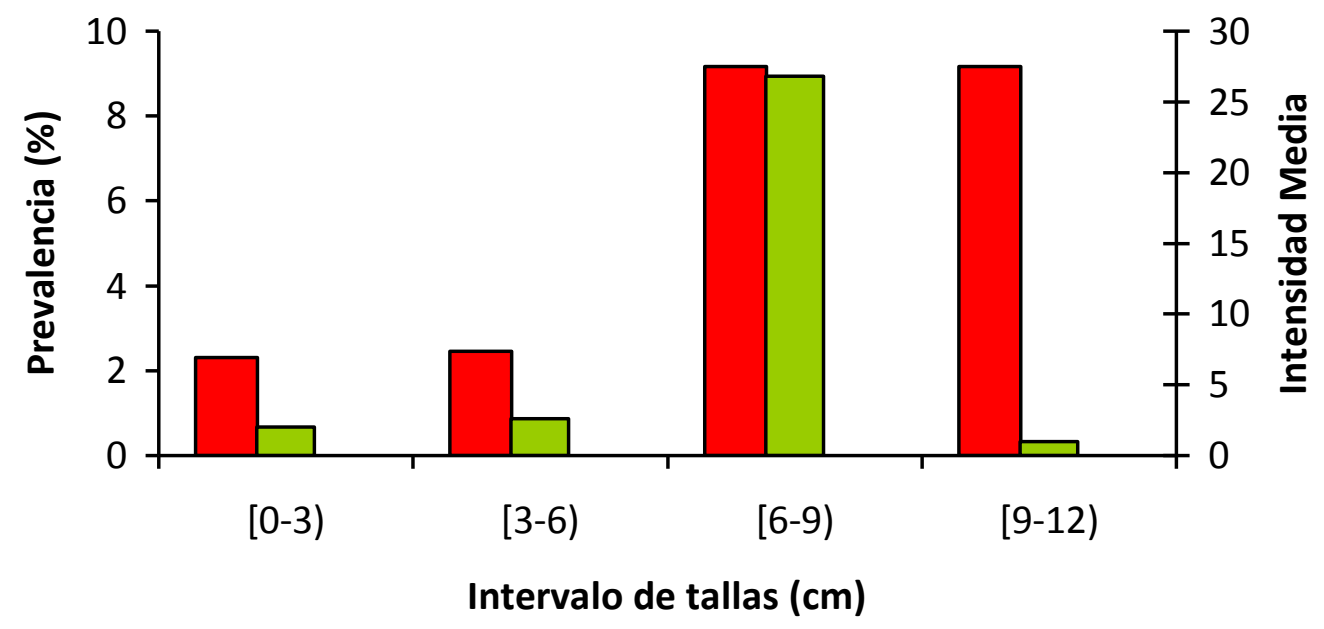

Figura 36. Intensidad media $v s$ prevalencia de Aelurostrongylus abstrusus en relación a los intervalos de talla de Lisschatina fulica.

\section{Phyllocaulis variegatus}

Para el caso de Brachylaima sp. los parámetros poblacionales fueron: $\mathrm{P}=20,9 \%$, $\mathrm{AM}=2,43$ y $\mathrm{IM}=11,64$. La tabla 7 y figura 37 muestran la prevalencia, intensidad media y abundancia media de Brachylaima sp. en relación a los intervalos de tallas de 
P. variegatus. Se observó una correlación positiva y significativa entre la prevalencia de infección, intensidad media y el tamaño del hospedador $(\boldsymbol{r} \boldsymbol{s}=\mathbf{0 , 5 1})$. La intensidad de infección varió de 0-21. La mayor intensidad media de infección se observó en la talla [6-9) $\mathrm{cm}$.

Los parámetros poblacionales para Strongyluris sp. fueron: $\mathrm{P}=3 \%, \mathrm{AM}=0.12 \mathrm{e}$ $\mathrm{IM}=4$. La tabla 8 y figura 38 muestra los parámetros ecológicos de Strongyluris sp. en relación a los intervalos de talla de $P$. variegatus. Se observó una correlación positiva y significativa entre la prevalencia, intensidad media de infección y el tamaño del hospedador $(\boldsymbol{r s}=\mathbf{0 , 9 9})$. La intensidad de infección varió de 0 a 5 . La mayor intensidad media de infección se observó en la talla [6-9) cm. 
Tabla 7. Parámetros ecológicos de Brachylaima sp. en Phyllocaulis variegatus por intervalo de talla y bimestres.

\begin{tabular}{|c|c|c|c|c|c|c|c|}
\hline $\begin{array}{c}\text { Intervalos de } \\
\text { tamaño }\end{array}$ & Oct-Nov 2013 & Feb-Mar 2014 & Jun-Jul 2014 & Oct-Nov 2014 & Feb-Mar 2015 & Oct-Nov 2015 & SubTotal \\
\hline \multirow{4}{*}{$\begin{array}{c}1 \\
{[0-3 \mathrm{~cm})}\end{array}$} & $\mathbf{n}=2$ & $\mathbf{n}=0$ & $\mathbf{n}=0$ & $\mathbf{n}=0$ & $\mathbf{n}=0$ & $\mathbf{n}=0$ & \multirow{4}{*}{$\begin{array}{c}\mathbf{N}=2 \\
\mathbf{P}=0 \\
\mathbf{I M}=0 \\
\mathbf{A M}=0\end{array}$} \\
\hline & $\mathbf{P}=0$ & $\mathbf{P}=0$ & $\mathbf{P}=0$ & $\mathbf{P}=0$ & $\mathbf{P}=0$ & $\mathbf{P}=0$ & \\
\hline & $\mathbf{I} \mathbf{M}=0$ & $\mathbf{I} \mathbf{M}=0$ & $\mathbf{I M}=0$ & $\mathbf{I} \mathbf{M}=0$ & $\mathbf{I} \mathbf{M}=0$ & $\mathbf{I M}=0$ & \\
\hline & $\mathbf{A M}=0$ & $\mathbf{A M}=0$ & $\mathbf{A M}=0$ & $\mathbf{A M}=0$ & $\mathbf{A M}=0$ & $\mathbf{A M}=0$ & \\
\hline \multirow{4}{*}{$\begin{array}{c}2 \\
{[3-6 \mathrm{~cm})}\end{array}$} & $\mathbf{n}=21$ & $\mathbf{n}=7$ & $\mathbf{n}=0$ & $\mathbf{n}=2$ & $\mathbf{n}=2$ & $\mathbf{n}=3$ & \multirow{4}{*}{$\begin{aligned} \mathbf{N} & =35 \\
\mathbf{P} & =20 \\
\mathbf{I M} & =8.85 \\
\mathbf{A M} & =1.77\end{aligned}$} \\
\hline & $\mathbf{P}=28,5$ & $\mathbf{P}=14,2$ & $\mathbf{P}=0$ & $\mathbf{P}=0$ & $\mathbf{P}=0$ & $\mathbf{P}=0$ & \\
\hline & $\mathbf{I M}=7,3$ & $\mathbf{I M}=18$ & $\mathbf{I M}=0$ & $\mathbf{I M}=0$ & $\mathbf{I M}=0$ & $\mathbf{I M}=0$ & \\
\hline & $\mathbf{A M}=2$ & $\mathbf{A M}=2,5$ & $\mathbf{A M}=0$ & $\mathbf{A M}=0$ & $\mathbf{A M}=0$ & $\mathbf{A M}=0$ & \\
\hline \multirow{4}{*}{$\begin{array}{c}3 \\
{[6-9 \mathrm{~cm})}\end{array}$} & $\mathbf{n}=7$ & $\mathbf{n}=7$ & $\mathbf{n}=1$ & $\mathbf{n}=1$ & $\mathbf{n}=2$ & $\mathbf{n}=8$ & \multirow{4}{*}{$\begin{aligned} \mathbf{N} & =26 \\
\mathbf{P} & =23 \\
\mathbf{I M} & =21,5 \\
\mathbf{A M} & =4,96\end{aligned}$} \\
\hline & $\mathbf{P}=0$ & $\mathbf{P}=14,2$ & $\mathbf{P}=100$ & $\mathbf{P}=0$ & $\mathbf{P}=0$ & $\mathbf{P}=37,5$ & \\
\hline & $\mathbf{I M}=0$ & $\mathbf{I M}=50$ & $\mathbf{I M}=40$ & $\mathbf{I} \mathbf{M}=0$ & $\mathbf{I M}=0$ & $\mathbf{I M}=3$ & \\
\hline & $\mathbf{A M}=0$ & $\mathbf{A M}=7,1$ & $\mathbf{A M}=40$ & $\mathbf{A M}=0$ & $\mathbf{A M}=0$ & $\mathbf{A M}=1,1$ & \\
\hline \multirow{4}{*}{$\begin{array}{c}4 \\
{[9-12 \mathrm{~cm})}\end{array}$} & $\mathbf{n}=0$ & $\mathbf{n}=2$ & $\mathbf{n}=0$ & $\mathbf{n}=1$ & $\mathbf{n}=0$ & $\mathbf{n}=1$ & \multirow{4}{*}{$\begin{array}{c}\mathbf{N}=\mathbf{4} \\
\mathbf{P}=25 \\
\mathbf{I M}=2 \\
\mathbf{A M}=0,5\end{array}$} \\
\hline & $\mathbf{P}=0$ & $\mathbf{P}=50$ & $\mathbf{P}=0$ & $\mathbf{P}=0$ & $\mathbf{P}=0$ & $\mathbf{P}=0$ & \\
\hline & $\mathbf{I M}=0$ & $\mathbf{I M}=2$ & $\mathbf{I} \mathbf{M}=0$ & $\mathbf{I M}=0$ & $\mathbf{I M}=0$ & $\mathbf{I M}=0$ & \\
\hline & $\mathbf{A M}=0$ & $\mathbf{A M}=1$ & $\mathbf{A M}=0$ & $\mathbf{A M}=0$ & $\mathbf{A M}=0$ & $\mathbf{A M}=0$ & \\
\hline Subtotal & $\begin{array}{c}\mathbf{N}=30 \\
\mathbf{P}=23,33 \\
\mathbf{I M}=6,28 \\
\mathbf{A M}=1,46\end{array}$ & $\begin{array}{c}\mathbf{N}=16 \\
\mathbf{P}=18,75 \\
\mathbf{I M}=23,33 \\
\mathbf{A M}=4,37\end{array}$ & $\begin{array}{c}\mathbf{N}=1 \\
\mathbf{P}=100 \\
\mathbf{I M}=40 \\
\mathbf{A M}=40\end{array}$ & $\begin{array}{c}\mathbf{N}=4 \\
\mathbf{P}=0 \\
\mathbf{I M}=0 \\
\mathbf{A M}=0\end{array}$ & $\begin{array}{c}\mathbf{N}=4 \\
\mathbf{P}=0 \\
\mathbf{I M}=0 \\
\mathbf{A M}=0\end{array}$ & $\begin{array}{c}\mathbf{N}=12 \\
\mathbf{P}=8,33 \\
\mathbf{I M}=5 \\
\mathbf{A M}=0,41\end{array}$ & $\begin{array}{c}\text { TOTAL } \\
67\end{array}$ \\
\hline
\end{tabular}

$\mathbf{P}=$ Prevalencia $; \mathbf{I M}=$ Intensidad media; $\mathbf{A M}=$ Abundancia media . 
Tabla 8. Parámetros ecológicos de Strongyluris sp. en Phyllocaulis variegatus por intervalo de talla y bimestres.

\begin{tabular}{|c|c|c|c|c|c|c|c|}
\hline $\begin{array}{c}\text { Intervalos de } \\
\text { tamaño }\end{array}$ & Oct-Nov 2013 & Feb-Mar 2014 & Jun-Jul 2014 & Oct-Nov 2014 & Feb-Mar 2015 & Oct-Nov 2015 & SubTotal \\
\hline \multirow{4}{*}{$\begin{array}{c}1 \\
{[0-3 \mathrm{~cm})}\end{array}$} & $\mathbf{n}=2$ & $\mathbf{n}=0$ & $\mathbf{n}=0$ & $\mathbf{n}=0$ & $\mathbf{n}=0$ & $\mathbf{n}=0$ & \multirow{4}{*}{$\begin{array}{c}\mathbf{N}=\mathbf{2} \\
\mathbf{P}=0 \\
\mathbf{I M}=0 \\
\mathbf{A M}=0\end{array}$} \\
\hline & $\mathbf{P}=0$ & $\mathbf{P}=0$ & $\mathbf{P}=0$ & $\mathbf{P}=0$ & $\mathbf{P}=0$ & $\mathbf{P}=0$ & \\
\hline & $\mathbf{I M}=0$ & $\mathbf{I} \mathbf{M}=0$ & $\mathbf{I M}=0$ & $\mathbf{I M}=0$ & $\mathbf{I} \mathbf{M}=0$ & $\mathbf{I M}=0$ & \\
\hline & $\mathbf{A M}=0$ & $\mathbf{A M}=0$ & $\mathbf{A M}=0$ & $\mathbf{A M}=0$ & $\mathbf{A M}=0$ & $\mathbf{A M}=0$ & \\
\hline \multirow{4}{*}{$\begin{array}{c}2 \\
{[3-6 \mathrm{~cm})}\end{array}$} & $\mathbf{n}=21$ & $\mathbf{n}=7$ & $\mathbf{n}=0$ & $\mathbf{n}=2$ & $\mathbf{n}=2$ & $\mathbf{n}=3$ & \multirow{4}{*}{$\begin{array}{c}\mathbf{N}=35 \\
\mathbf{P}=2.85 \\
\mathbf{I M}=3 \\
\mathbf{A M}=0.08\end{array}$} \\
\hline & $\mathbf{P}=4,16$ & $\mathbf{P}=0$ & $\mathbf{P}=0$ & $\mathbf{P}=0$ & $\mathbf{P}=0$ & $\mathbf{P}=0$ & \\
\hline & $\mathbf{I M}=3$ & $\mathbf{I M}=0$ & $\mathbf{I} \mathbf{M}=0$ & $\mathbf{I M}=0$ & $\mathbf{I} \mathbf{M}=0$ & $\mathbf{I} \mathbf{M}=0$ & \\
\hline & $\mathbf{A M}=0,1$ & $\mathbf{A M}=0$ & $\mathbf{A M}=0$ & $\mathbf{A M}=0$ & $\mathbf{A M}=0$ & $\mathbf{A M}=0$ & \\
\hline \multirow{4}{*}{$\begin{array}{c}3 \\
{[6-9 \mathrm{~cm})}\end{array}$} & $\mathbf{n}=7$ & $\mathbf{n}=7$ & $\mathbf{n}=1$ & $\mathbf{n}=1$ & $\mathbf{n}=2$ & $\mathbf{n}=8$ & \multirow{4}{*}{$\begin{array}{c}\mathbf{N}=26 \\
\mathbf{P}=3,84 \\
\mathbf{I M}=5 \\
\mathbf{A M}=0,19\end{array}$} \\
\hline & $\mathbf{P}=0$ & $\mathbf{P}=0$ & $\mathbf{P}=100$ & $\mathbf{P}=0$ & $\mathbf{P}=0$ & $\mathbf{P}=12,5$ & \\
\hline & $\mathbf{I} \mathbf{M}=0$ & $\mathbf{I} \mathbf{M}=0$ & $\mathbf{I M}=40$ & $\mathbf{I} \mathbf{M}=0$ & $\mathbf{I} \mathbf{M}=0$ & $\mathbf{I M}=5$ & \\
\hline & $\mathbf{A M}=0$ & $\mathbf{A M}=0$ & $\mathbf{A M}=40$ & $\mathbf{A M}=0$ & $\mathbf{A M}=0$ & $\mathbf{A M}=0,6$ & \\
\hline \multirow{4}{*}{$\begin{array}{c}4 \\
{[9-12 \mathrm{~cm})}\end{array}$} & $\mathbf{n}=0$ & $\mathbf{n}=2$ & $\mathbf{n}=0$ & $\mathbf{n}=1$ & $\mathbf{n}=0$ & $\mathbf{n}=1$ & \multirow{4}{*}{$\begin{array}{c}\mathbf{N}=4 \\
\mathbf{P}=0 \\
\mathbf{I M}=0 \\
\mathbf{A M}=0\end{array}$} \\
\hline & $\mathbf{P}=0$ & $\mathbf{P}=0$ & $\mathbf{P}=0$ & $\mathbf{P}=0$ & $\mathbf{P}=0$ & $\mathbf{P}=0$ & \\
\hline & $\mathbf{I} \mathbf{M}=0$ & $\mathbf{I} \mathbf{M}=0$ & $\mathbf{I} \mathbf{M}=0$ & $\mathbf{I} \mathbf{M}=0$ & $\mathbf{I M}=$ & $\mathbf{I M}=0$ & \\
\hline & $\mathbf{A M}=0$ & $\mathbf{A M}=0$ & $\mathbf{A M}=0$ & $\mathbf{A M}=0$ & $\mathbf{A M}=0$ & $\mathbf{A M}=0$ & \\
\hline Subtotal & $\begin{array}{c}\mathbf{N}=30 \\
\mathbf{P}=3,33 \\
\mathbf{I M}=3 \\
\mathbf{A M}=0,1\end{array}$ & $\begin{array}{c}\mathbf{N}=16 \\
\mathbf{P}=0 \\
\mathbf{I M}=0 \\
\mathbf{A M}=0\end{array}$ & $\begin{array}{c}\mathbf{N}=1 \\
\mathbf{P}=0 \\
\mathbf{I M}=0 \\
\mathbf{A M}=0\end{array}$ & $\begin{array}{c}\mathbf{N}=\mathbf{4} \\
\mathbf{P}=0 \\
\mathbf{I M}=0 \\
\mathbf{A M}=0\end{array}$ & $\begin{array}{c}\mathbf{N}=\mathbf{4} \\
\mathbf{P}=0 \\
\mathbf{I M}=0 \\
\mathbf{A M}=0\end{array}$ & $\begin{array}{c}\mathbf{N}=\mathbf{1 2} \\
\mathbf{P}=33,3 \\
\mathbf{I M}=1,25 \\
\mathbf{A M}=0,41\end{array}$ & $\begin{array}{c}\text { TOTAL } \\
67\end{array}$ \\
\hline
\end{tabular}

$\mathbf{P}=$ Prevalencia $\mathbf{I M}=$ Intensidad media; $\mathbf{A M}=$ Abundancia media . 
Prevalencia

Intensidad Media

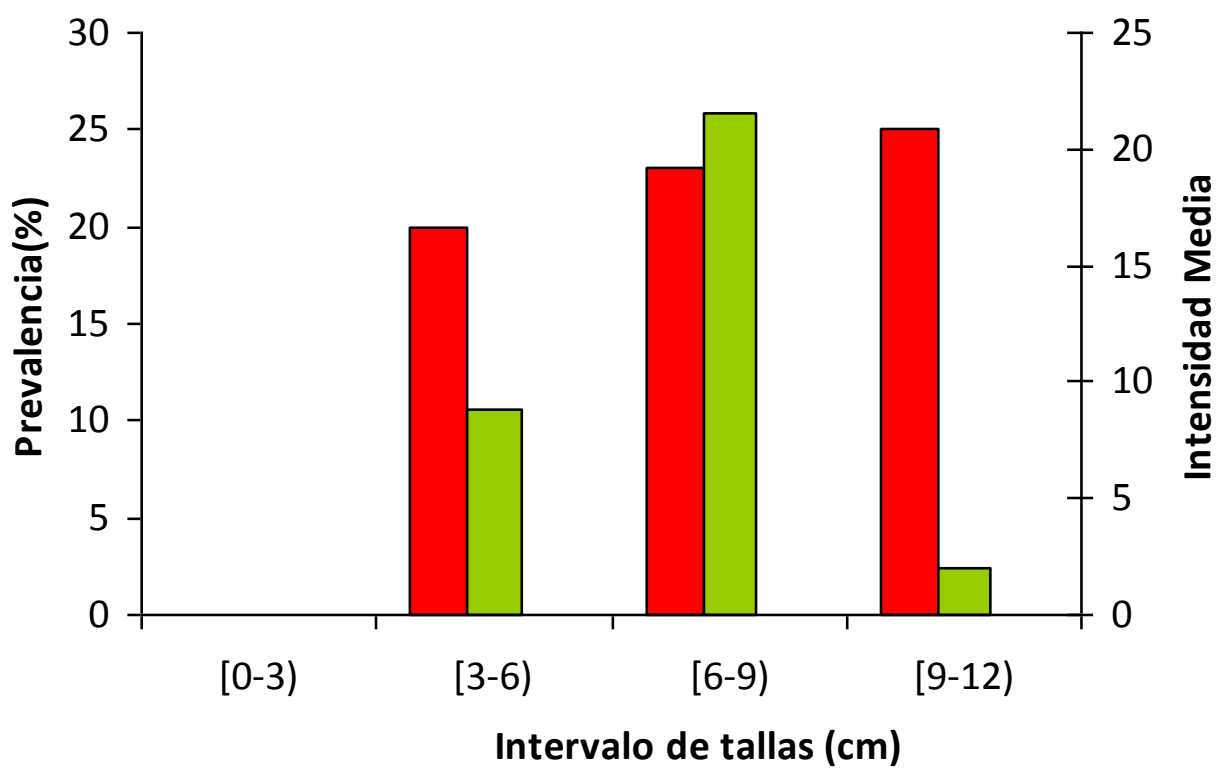

Figura 37. Intensidad media $v s$ prevalencia de Brachylaima sp. en relación a los intervalos de talla $P$. variegatus.

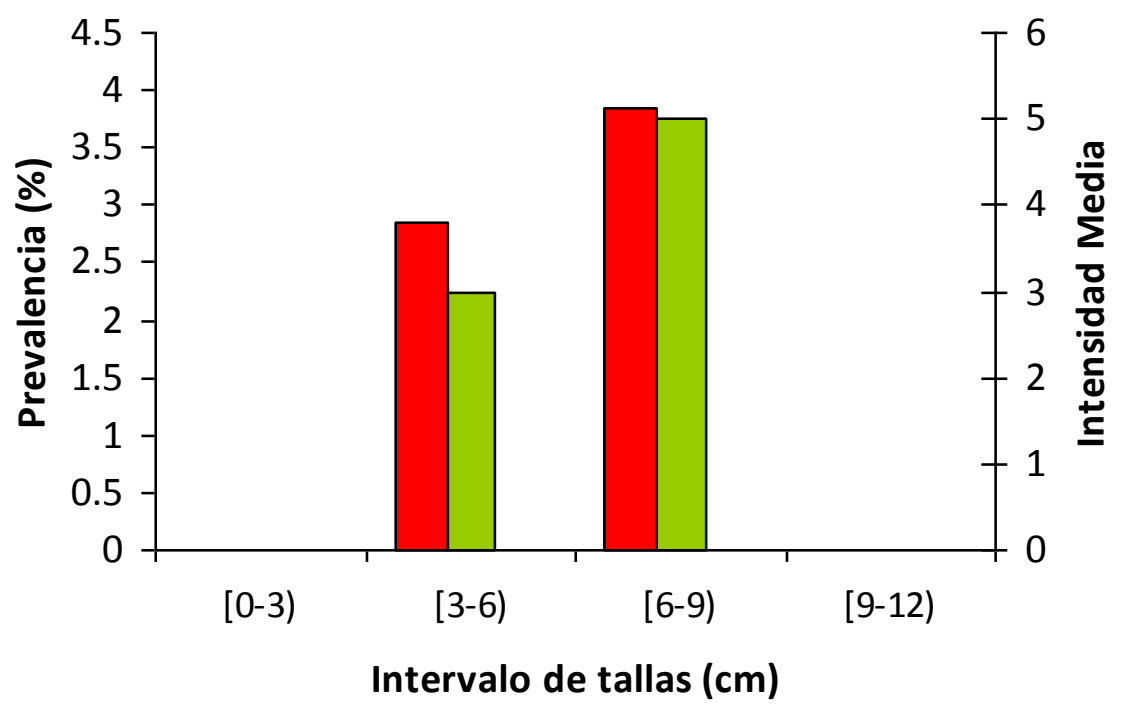

Figura 38. Intensidad media vs prevalencia de Strongyluris sp. en relación a los intervalos de talla de $P$. variegatus. 


\subsection{Influencia de los componentes temporal y ambiental en la distribución parasitaria}

\section{Lissacathina fulica}

La figura 39 muestra la prevalencia e intensidad media de Strongyluris sp. en relación a cada bimestre. No se observó correlación entre la prevalencia, intensidad media de infección y los bimestres $(\boldsymbol{r} \boldsymbol{s}=\mathbf{0 , 2 6})$. La intensidad de infección varió de 0 a 19. La mayor intensidad media de infección se observó en el bimestre Junio-Julio 2015, seguida de febrero-marzo 2014.

Para el caso de A. abstrusus no se observó correlación entre la prevalencia, intensidad media y los bimestres ( $r \mathbf{s}=\mathbf{0 , 0 6}$ ) (Figura 40). En el caso de la intensidad media, esta varió de 0 a 45, siendo febrero-marzo 2015 el bimestre con mayor intensidad media de infección.

Prevalencia

Intensidad Media

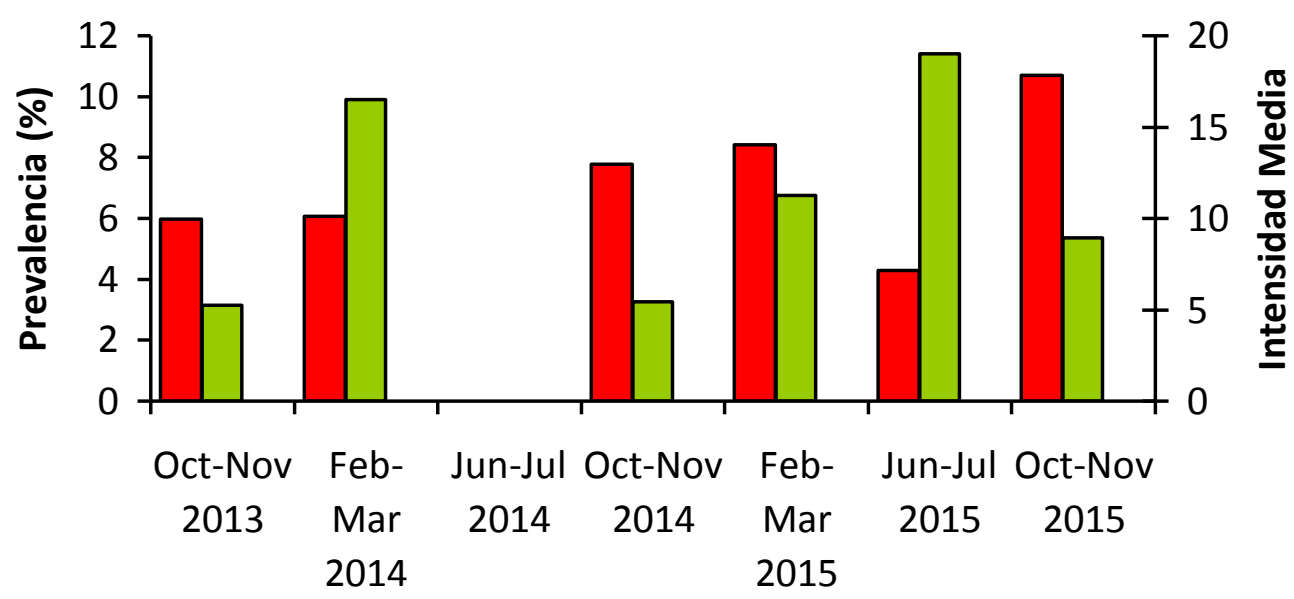

Bimestre

Figura 39. Intensidad media y prevalencia de Strongyluris sp. en L. fulica. 


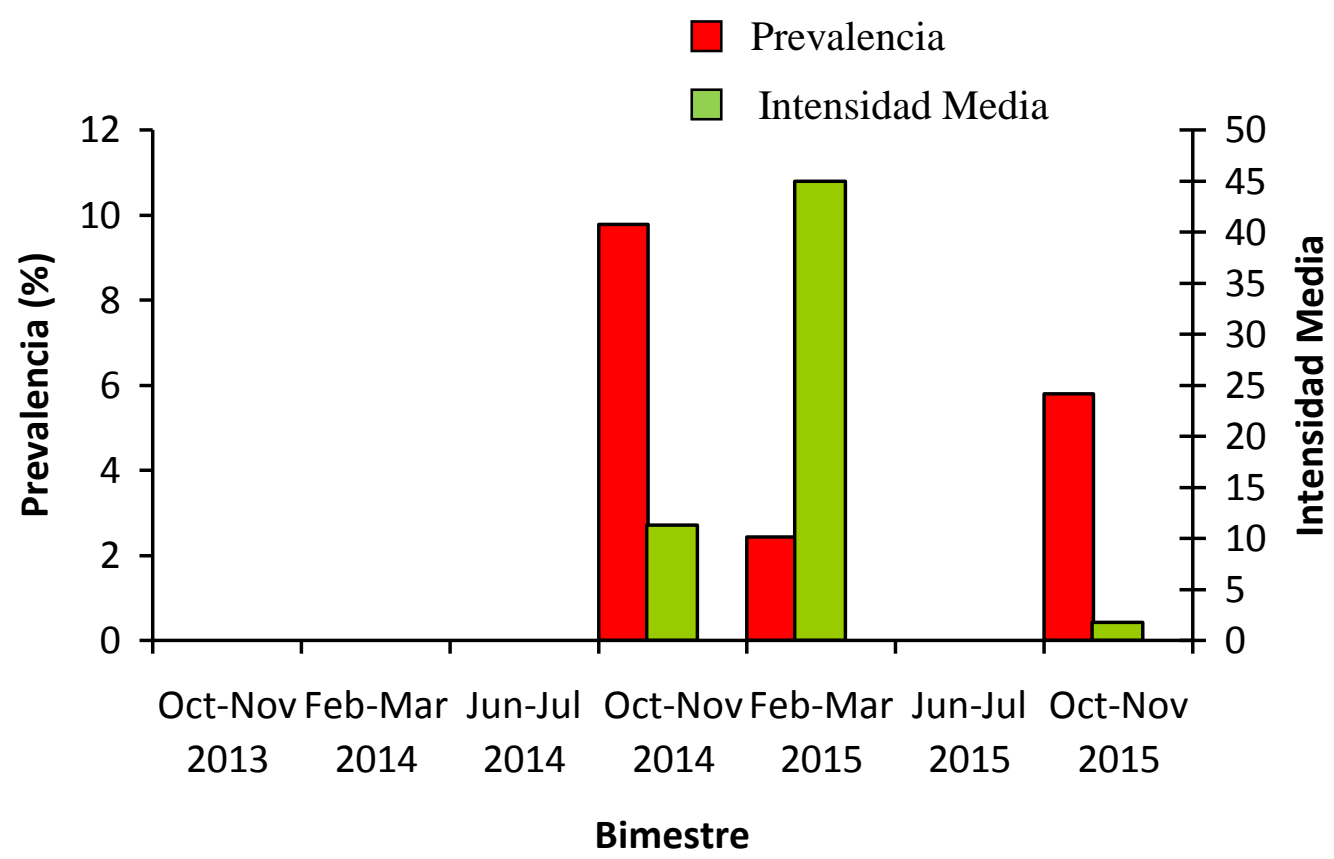

Figura 40. Intensidad media y prevalencia de Aelurostrongylus abstrusus en L. fulica.

\section{Phyllocaulis variegatus}

En la figura 41 se observan la prevalencia e intensidad media de Brachylaima sp. en relación a cada bimestre. La intensidad media varió de 0 a 40, siendo mayor en el bimestre junio-julio 2014. Se observó una correlación positiva entre la prevalencia, la intensidad media y los bimestres $(\boldsymbol{r s}=\mathbf{0 , 9 9})$.

En el caso de Strongyluris sp. la intensidad media varió de 0 a 3, el bimestre en el que se observó la mayor intensidad media fue octubre-noviembre 2015 (Figura 42). Se observó una correlación positiva aunque poco significativa entre la prevalencia, la intensidad media y los bimestres $(\boldsymbol{r s}=\mathbf{0 , 3 1})$. 


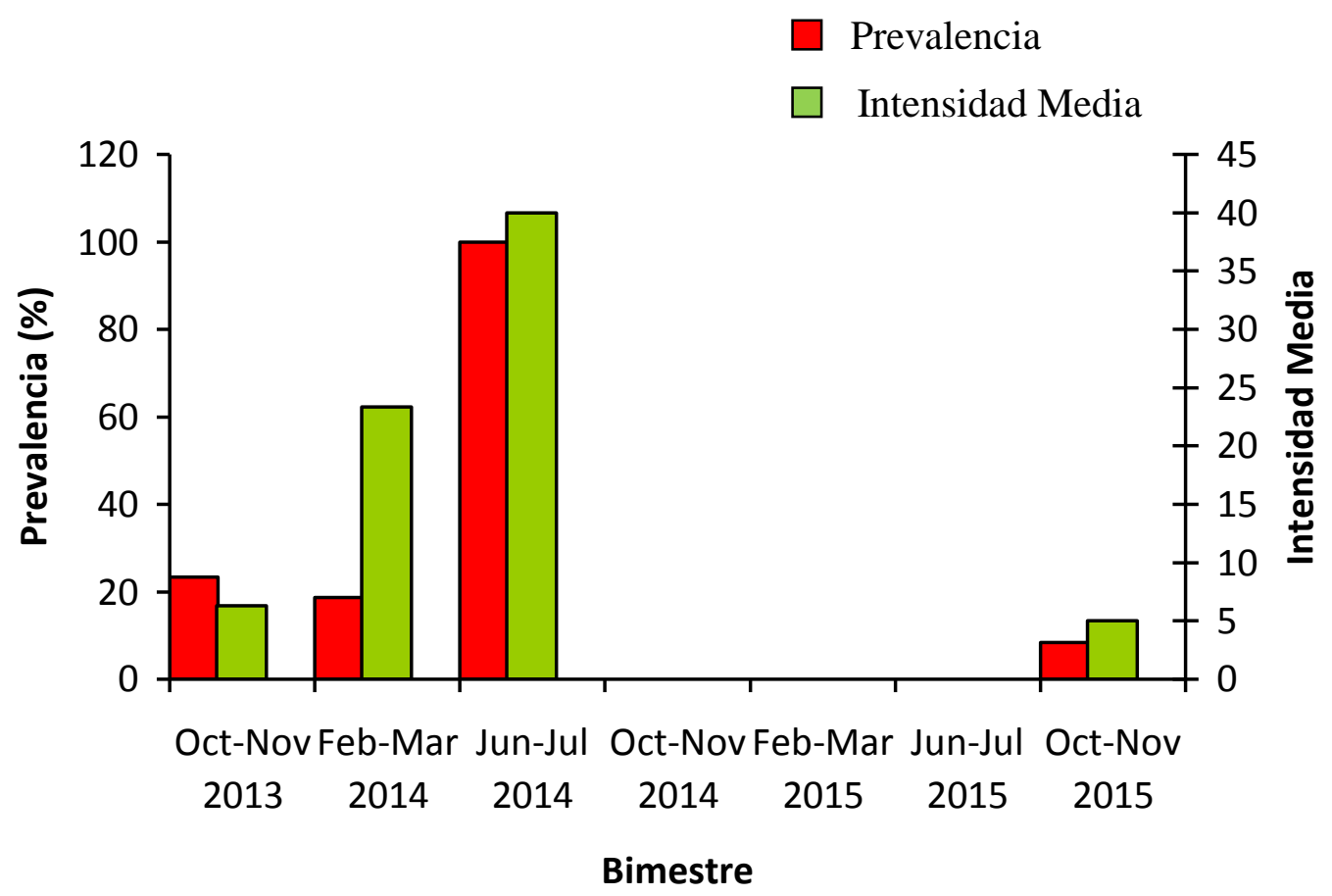

Figura 41. Intensidad media y prevalencia de Brachylaima sp. en P. variegatus.

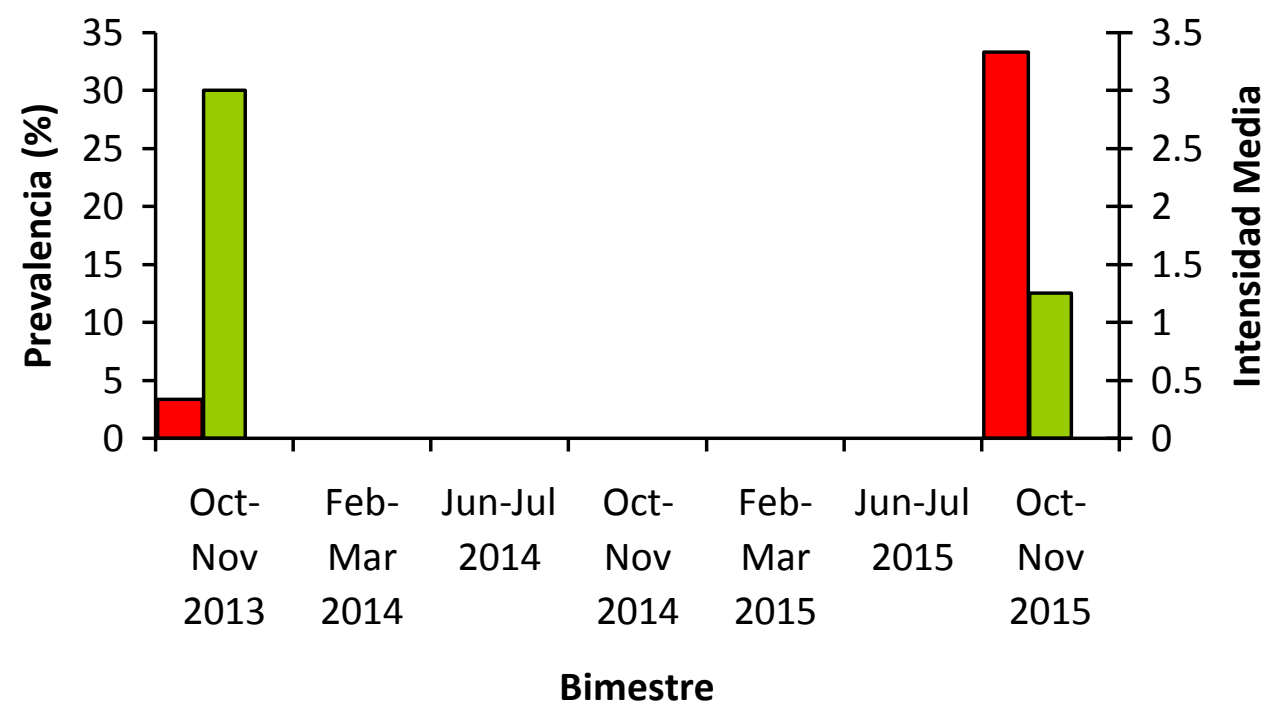

Figura 42. Intensidad media y prevalencia de Strongyluris sp. en P. variegatus. 


\section{DISCUSIÓN}

\section{Relación entre prevalencia e intensidad media y el tamaño del hospedador}

Diversos estudios mostraron que la intensidad de infección puede estar condicionada por el tamaño del hospedador, dado que los hospedadores de mayor tamaño proveen más recursos (por ejemplo, espacio y disponibilidad de nutrientes) para el crecimiento de los parásitos (Poulin \& Morand, 2004). En el presente trabajo se observó un incremento de la prevalencia de Strongyluris sp. con el incremento del tamaño de L. fulica. Además, los caracoles de mayor tamaño albergaron más parásitos que los caracoles más pequeños. Para el caso de A. abstrusus también se observó un aumento de la prevalencia e intensidad media de infección con el correspondiente incremento del tamaño de Lissachatina fulica.

Similares resultados fueron observados para Brachylaima sp. y Strongyluris sp. en $P$. variegatus, en los cuales la prevalencia e intensidad media mostraron estrecha relación con el tamaño del hospedador. Las babosas de mayor tamaño $(6-9 \mathrm{~cm})$ presentaron mayor número de parásitos.

En este sentido, los resultados de este estudio muestran un incremento de la prevalencia e intensidad de infección por especie parasita analizada, con el correspondiente incremento del tamaño de los hospedadores.

\section{Influencia de los componentes temporal y ambiental en la distribución parasitaria}

Los endoparásitos están expuestos indirectamente a los efectos de las condiciones externas por el periodo de tiempo en el que permanecen dentro del hospedador. Factores extrínsecos como la temperatura pueden tener un impacto en ellos, modificando la fisiología de los hospedadores, y causando un efecto indirecto sobre los parásitos. Esta situación es más perjudicial cuando el hospedador no posee una regulación interna (efecto buffer) de las condiciones externas, como es el caso de los hospedadores invertebrados (Poulin, 2007).

Los resultados obtenidos para Strongyluris sp. y A. abstrusus en L. fulica, muestran que no existiría una asociación entre las variables ambientales y la distribución parasitaria. Teniendo en cuenta que $L$ fulica logra adaptarse a las altas temperaturas del ambiente mediante períodos de estivación, favoreciendo su 
homeostasis, no existiría una influencia directa del ambiente sobre la distribución parasitaria.

Resultados similares pudieron observarse para Strongyluris sp. y Brachylaima sp. en $P$. variegatus, en los cuales no se observó relación entre las variables ambientales y los parásitos asociados. Phyllocaulis variegatus a diferencia de L. fulica presenta mecanismos de adaptaciones más sencillas tales como ocultarse en sitios húmedos, hábitos nocturnos, envolverse en segregación mucosa que le permitiría tolerar las altas temperaturas del ambiente y no afectar la distribución parasitaria. 


\section{Conclusiones}

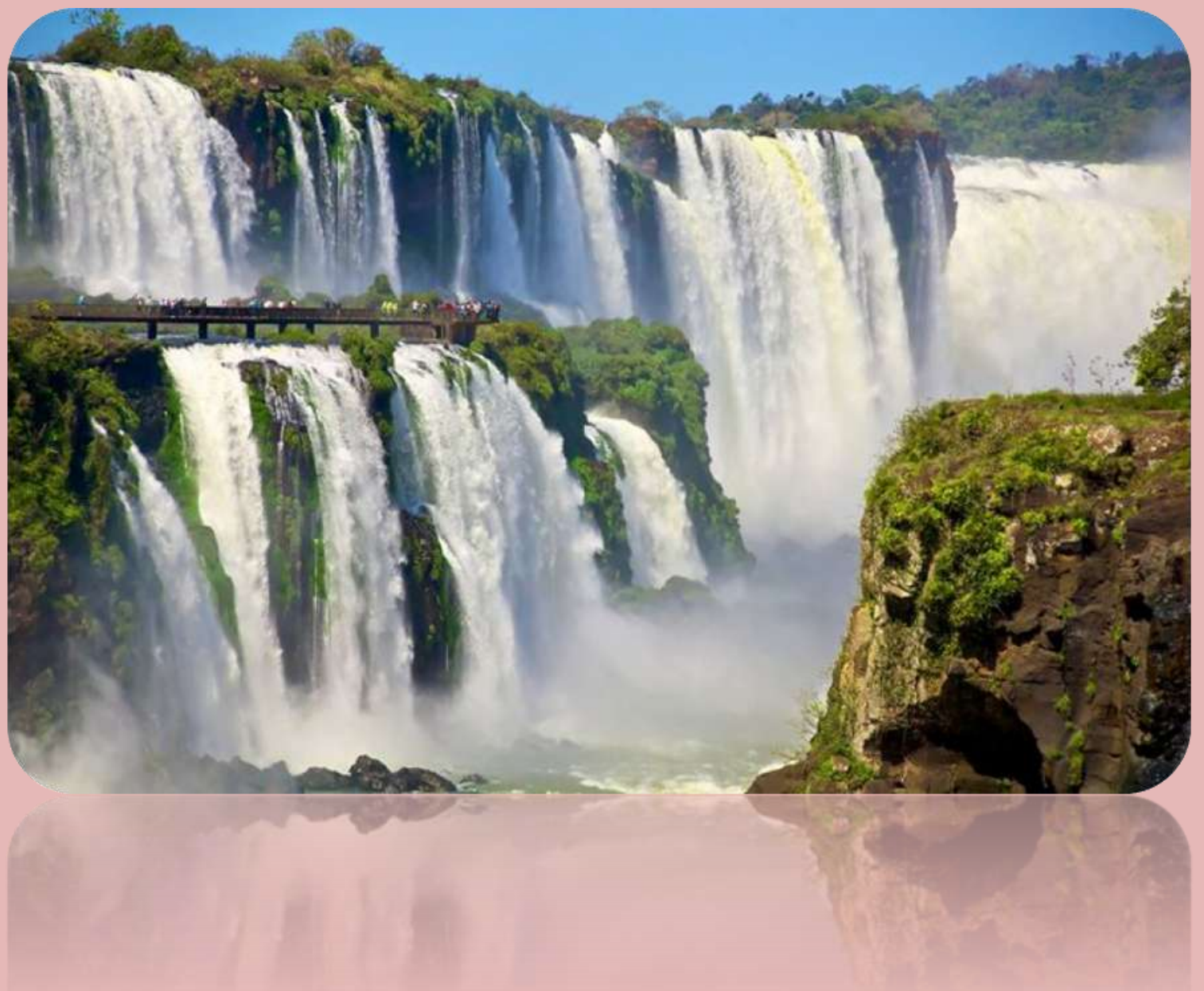




\section{CONCLUSIONES}

En este trabajo se evaluó la distribución, dispersión y parasitofauna del caracol gigante africano Lissachatina fulica (Achatinidae) en la ciudad de Puerto Iguazú, Misiones, desde su introducción hasta la actualidad, y de las babosas nativas Phyllocaulis variegatus y Latipes erinaceus (Veronicellidae), con el fin de determinar su rol en la transmisión de helmintos parásitos, con especial énfasis en los nematodes Metastrongylidae. El muestreo se llevó a cabo principalmente en el área urbana, aunque también se analizaron zonas no pobladas. Los resultados obtenidos de esta investigación permiten alcanzar las siguientes conclusiones:

\section{En relación a los hospedadores:}

- $\quad$ La distribución espacial de L. fulica en la ciudad de Puerto Iguazú, desde su introducción (2010) hasta la actualidad se incrementó, encontrándose durante este estudio nuevas áreas focos, distantes $3 \mathrm{~km}$ del área foco inicial. La propagación puede atribuirse a varias causas: a) influencia antrópica (remoción y traslado de tierra), b) utilización de ejemplares para pesca, c) ausencia de enemigos naturales, d) alta tasa de reproducción, e) plasticidad para adaptarse a condiciones ambientales desfavorables.

- La distribución espacial de las babosas de la familia Veronicellidae, se redujo a lo largo de los años de muestreo. El descenso y/o ausencia del número de babosas pueden atribuirse a: a) comportamiento depredador de L. fulica sobre los Veronicelidos, b) uso de molusquicidas ya que son consideradas plaga para las huertas orgánicas, c) dificultad para su observación durante los horarios de muestreo por sus hábitos nocturnos.

- Los factores ambientales influyen en el tamaño corporal de L. fulica, observándose una significativa asociación entre las tallas más pequeñas $[0-3 \mathrm{~cm}$ ) y escasas precipitaciones-bajas temperaturas (período de hibernación), y entre las tallas más grandes $\left[6-12 \mathrm{~cm}\right.$ ) con temperaturas máximas promedio 24-21 $\mathrm{C}^{\circ}, \mathrm{y}$ precipitaciones continuas (períodos postestivación).

- En relación a las babosas Veronicellidae las tallas intermedias [3-9 cm) fueron las más abundantes en el bimestre octubre-noviembre con el incremento de las lluvias y 
el descenso de las temperaturas, aspecto importante para organismos desnudos que respiran a través de su tegumento.

- Los mapas predictivos de distribución de nicho ecológico, predicen que L. fulica en el 2050 se localizará por debajo de la línea del Ecuador (áreas coloreadas con color rojo y naranja), dispersándose a nuevas áreas que en la actualidad no poseen las características ambientales para su desarrollo. (Este, Oeste y Sur de Sudamérica, incluida la totalidad de Argentina). Sin embargo teniendo en cuenta el cambio climático global y la biología de L. fulica, no es posible afirmar que su distribución pueda alcanzar los límites predichos a partir del análisis de distribución de nichos ecológicos elaborado.

En relación a los parásitos:

- Se hallaron tres especies de helmintos parásitos en los hospedadores analizados: Brachylaima sp. (Digenea: Brachylaimidae), Strongyluris sp. (Nematoda: Heterakidae) y Aleurostrongylus abstrusus (Nematoda: Angiostrongylidae)

- La baja prevalencia e intensidad media de Strongyluris sp.y Aelurostrongylus abstrusus en L. fulica estaría relacionada con su reciente introducción en Puerto Iguazú.

- $\quad$ Entre las dos especies de Veronicelidos consideradas en este trabajo, solo $P$. variegatus se halló parasitado por Strongyluris sp y Brachylaima sp.

- La relación entre carga de parásitos y el tamaño de los hospedadores fue significativamente positiva, a mayor biomasa disponible mayor recurso para los parásitos.

- Las variables ambientales no influyen sobre la distribución de las especies parásitas. Probablemente los hospedadores desarrollaron estrategias que permitieron ajustar su fisiología, impidiendo que las condiciones externas influyan sobre su parasitofauna.

- No se observó asociación entre las variables ambientales y la distribución de Strongyluris sp. y A. abstrusus en $L$. fulica, en respuesta a la capacidad que muestra $L$ fulica en adaptarse a las altas temperaturas mediante periodos de estivación. 
Sobre la base de los resultados alcanzados y el progreso de esta línea de investigación se espera:

1) continuar con el monitoreo de L. fulica y su parasitofauna, teniendo en cuenta la cercanía a zonas con registros Angiostrongyliasis (enfermedad zoonótica).

2) ampliar el estudio a otros moluscos terrestres (e.g. Bulimulus sp., Bradybaena similaris) y dulceacuícolas (e.g. Pomacea sp) que puedan intervenir en el ciclo de vida de nematodes metastrongylidos de importancia sanitaria.

3) estudiar los ciclos de vida de los nematodes Metastrongyloidea mediante infestaciones experimentales para lograr identificar qué hospedadores intermediarios y definitivos son más susceptibles, identificando además las patologías asociadas a las diferentes especies. 

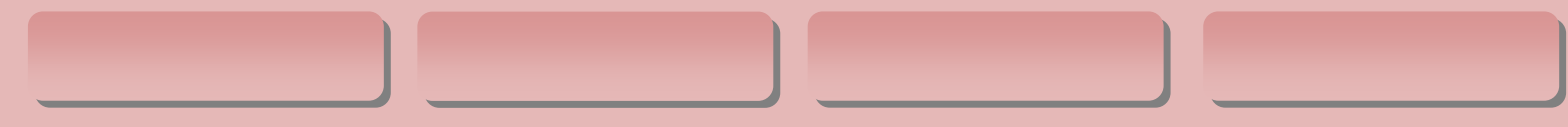

\section{Anexos}

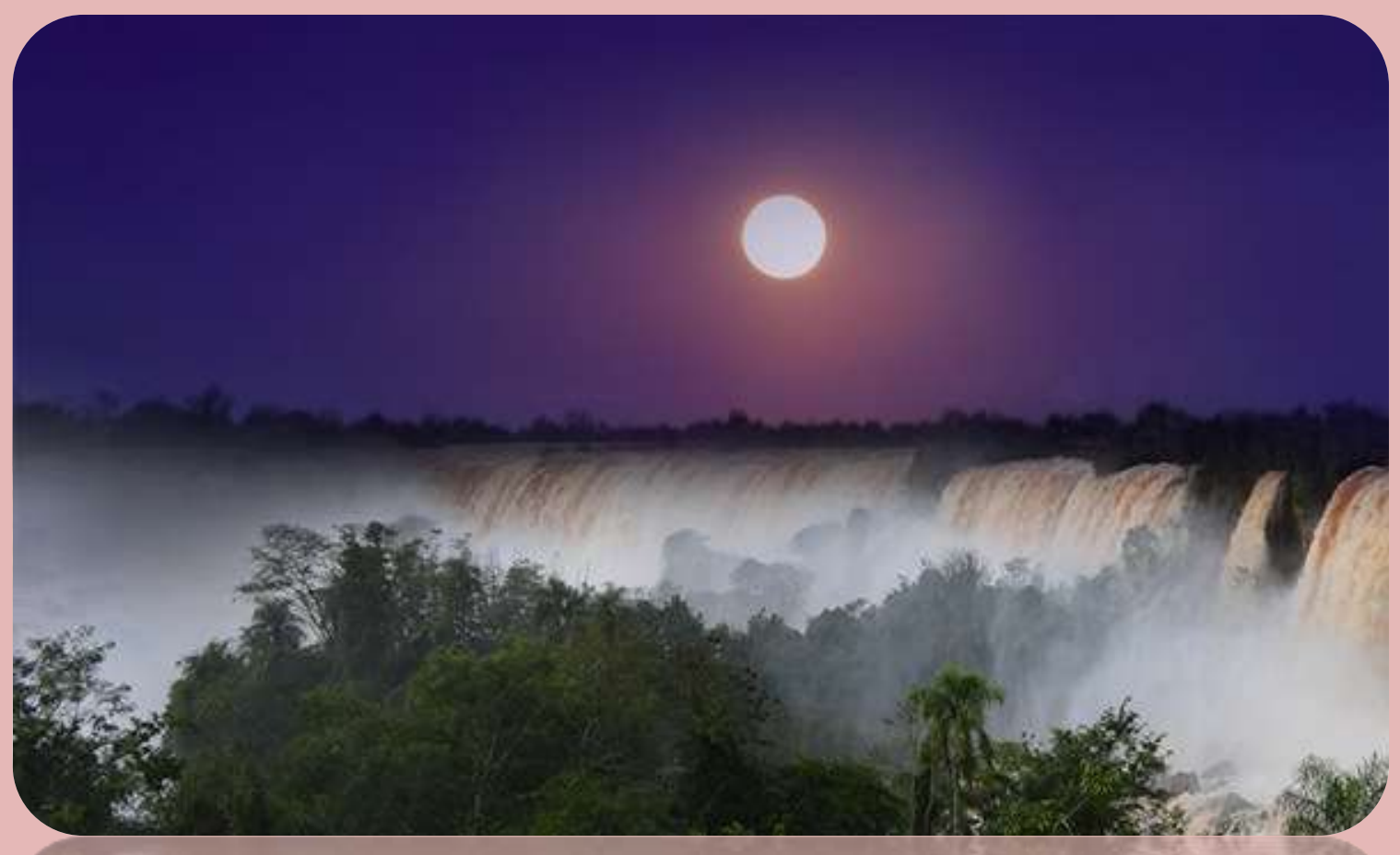




\section{Angiostrongylus cantonensis}

El ciclo de vida comienza cuando la hembra ovipone dentro de las arteriolas pulmonares de su HD, (Rattus rattus, Rattus norvergicus) donde eclosionan las larvas de primer estadio (L1), que luego pasan al interior de los alvéolos. Las larvas migran hacia la faringe para posteriormente ser deglutidas, pasando por el tracto gastrointestinal y ser finalmente eliminadas con las heces. Los caracoles terrestres que actúan como hospedadores intermediarios pueden infectarse por ingestión o penetración de las L1. Una vez dentro del HI la L1 muda dos veces dando lugar a la larva infectante (L3). Los HD se infectan al ingerir los HI parasitados con L3. Estas larvas penetran la pared intestinal, luego entran al torrente sanguíneo y unas horas después de haber sido ingeridas, llegan a la circulación pulmonar del corazón y se dispersan a varios otros órganos por la circulación de la sangre. Muchas llegan al cerebro y mudan nuevamente, dando lugar a la L4. La quinta muda se produce en el espacio subaracnoideo, donde la L5 migra a las arterias pulmonares para allí alcanzar su madurez sexual (Maldonado Jr et al., 2012).

El hombre es un hospedador accidental dentro del ciclo de $A$. cantonensis, ya que se infecta al consumir caracoles o babosa crudas, o a través del consumo de hospedadores paraténico tales como cangrejos o camarones de agua dulce. Otra forma de contagio es a través del manipuleo de los HI, ya que las larvas son eliminadas por las secreciones mucosas de los gasterópodos, al no tener hábitos higiénicos adecuados, las L3 son ingeridas por el hombre. La L3 puede llegar al cerebro y alojarse en las meninges. Debido a que el hombre no es el hospedador adecuado causa en él una enfermedad llamada meningoencefalitis eosinofílica (ME), que afecta al sistema nervioso central. Normalmente, la infección no mata a la víctima, pero el parasitismo puede ser lo suficientemente grave como para despertar una excesiva respuesta inmunológica en el hombre, que puede causar la muerte (Acha \& Szyfres, 2003; Caldeira et al., 2007, Maldonado Jr et al., 2012).

\section{Angiostrongylus costaricensis}

En el ciclo de vida de $A$. costaricensis los adultos se encuentran en las arterias mesentéricas del HD que son roedores tales como Sigmodon hispidus, Rattus rattus, Rattus norvergicus, Akodon montensis (Robles et al, 2016). Una vez allí la hembra ovipone y los huevos son transportados por el torrente sanguíneo, causando embolias en 
las arteriolas y capilares de la pared intestinal. Los huevos eclosionan liberando las L1 que penetran en la luz intestinal y salen al exterior con las heces. La L1 es ingerida o penetra activamente en el tejido de los HI tales como Phyllocaulis soleiformis (d'Orbigny), Phyllocaulis variegatus (Semper), Saranisula plebeia (Fischer, 1868), (Kaminsky et al., 1995; Ohlweiler et al., 2010). Una vez allí la L1 muda dos veces, hasta llegar a L3. Los HD se infectan consumiendo los HI o con comida contaminada con las secreciones mucosas de los HI. La L3 migra hacia la región ileocecal y penetra la pared del intestino para entrar en los vasos linfáticos, donde mudan dos veces más antes de migrar hacia las arterias mesentéricas, donde maduran sexualmente.

El hombre es un hospedador accidental dentro del ciclo de A. costaricensis, ya que se infecta al consumir al HI, o a través del consumo de comida mal lavada y contaminada. Otra forma de contagio es a través del manipuleo de los HI, ya que las larvas son eliminadas con las secreciones mucosas de los gasterópodos, y si no se tienen hábitos higiénicos adecuados, las L3 pueden ser ingeridas por el hombre. En los seres humanos, las L3 se encuentran en las vías mesentéricas, produciendo así una gran inflamación en la zona abdominal como consecuencia de las lesiones, o ulceraciones. La angiostrongyliasis abdominal no es una enfermedad mortal, pero puede llegar a ocasionar diferentes complicaciones en el ciego, el colon ascendente, el íleon y el apéndice cecal (Kamisnky et al., 1995, Maldonaldo Jr et al., 2012).

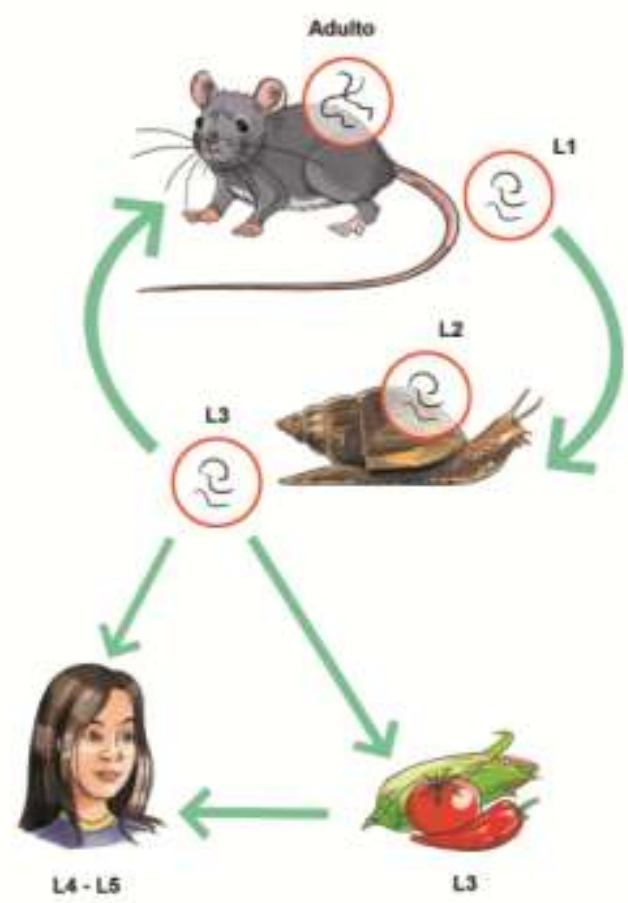

Ciclo de vida de Angiostrongylus cantonensis y Angiostrongylus costaricensis 


\section{Angiostrongylus vasorum y Aelurostrongylus abstrusus}

Ambos metastrongylidos de importancia veterinaria tienen un ciclo heteroxeno que incluye un HD que puede ser un cánido doméstico o silvestre en el primer caso, y félidos domésticos o silvestres en el último. El HI puede ser una babosa o un caracol terrestre tales como L. fulica, Saranisula marginata, Bradybaena similaris, Subulina octona (Silva Lucas, 2013) El HD ingiere al HI que porta la larva infectiva L3, la cual penetra la pared intestinal para alcanzar las arterias mesentéricas donde muda dos veces hasta llegar a L5. Esta larva viaja por el torrente sanguíneo hasta llegar al ventrículo derecho del corazón o a las arterias pulmonares donde alcanza la madurez sexual. La hembra deposita sus huevos en las ramificaciones de la arteria pulmonar y allí emerge la L1 que migra a los alvéolos, bronquiolos y bronquios, dirigiéndose a la tráquea para ser deglutida y llegar así al tubo digestivo y ser eliminada con las heces. La L1 llega al HI porque es consumida o por penetración activa en el tejido.

Esta enfermedad que produce obstrucciones respiratorias en félidos y cánidos.

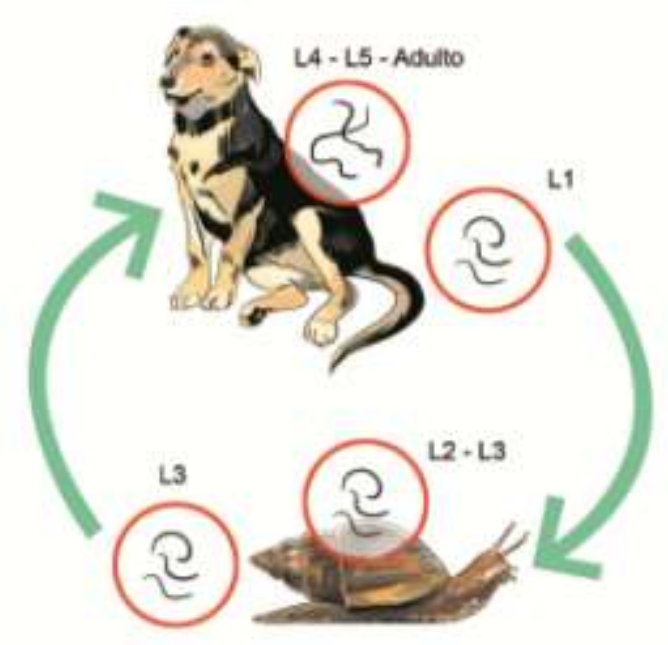

Ciclo de vida de Angiostrongylus vasorum 


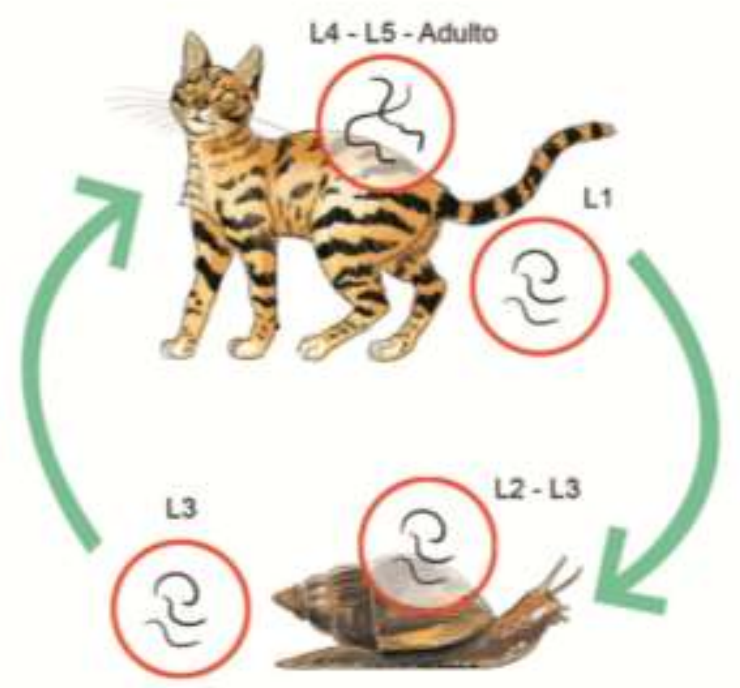

Ciclo de vida de Aelurostrongylus abstrusus 
ANEXO II. Registros de enfermedad, hospedadores definitivos e intermediarios de Angiostrongylus cantonensis.

\begin{tabular}{|c|c|c|c|c|c|c|}
\hline $\begin{array}{c}\text { Primer } \\
\text { hallazgo del } \\
\text { parásito adulto }\end{array}$ & Hospedador definitivo & $\begin{array}{c}\text { Hospedador } \\
\text { intermediario }\end{array}$ & $\begin{array}{l}\text { Primer registro } \\
\text { de enfermedad }\end{array}$ & $\begin{array}{c}\text { Hospedador accidental/ } \\
\text { paraténico }\end{array}$ & Sitio geográfico & Literatura consultada \\
\hline 1935 & $\begin{array}{l}\text { Rattus norvergicus } \\
\text { Rattus rattus }\end{array}$ & & & & Canton, China & Alicata, 1991 \\
\hline 1945 & $\begin{array}{l}\text { Rattus norvergicus } \\
\text { Rattus rattus }\end{array}$ & $\begin{array}{l}\text { Lissachatina fulica } \\
\text { Pomacea canaliculata }\end{array}$ & 1945 & $\begin{array}{l}\text { Sapos/Ranas/Peces }{ }^{1} \\
\text { Homo sapiens }\end{array}$ & Taiwán, China & Alicata, 1991 \\
\hline 1955 & $\begin{array}{l}\text { Rattus norvergicus } \\
\text { Rattus rattus }\end{array}$ & Deroceras laeve & 1980 & Homo sapiens & Australia* & Cross, 2007 \\
\hline 1960 & $\begin{array}{l}\text { Rattus rattus } \\
\text { Rattus norvegicus }\end{array}$ & $\begin{array}{l}\text { Lissachatina fulica } \\
\text { Veronicella cubensis } \\
\text { Parmarion martensis }\end{array}$ & 1960 & Homo sapiens & Hawaii* & Alicata, 1991 \\
\hline 1961 & Rattus norvergicus & Lissachatina fulica & 1984 & Homo sapiens & Tahiti* & Alicata, 1991 \\
\hline 1964 & Rattus norvergicus & $\frac{\text { Lissachatina fulica }}{\text { Pomacea canaliculata }}$ & 1979 & Homo sapiens & Islas Ryukyu, Japón & Alicata, 1991 \\
\hline 1965 & Rattus norvergicus & $\underline{\text { Lissachatina fulica }}$ & 1965 & Homo sapiens & Filipinas* & Alicata, 1991 \\
\hline 1968 & Rattus norvergicus & $\begin{array}{l}\text { Lissachatina fulica } \\
\text { Pomacea canaliculata }\end{array}$ & 1968 & Homo sapiens & Islas Hokkaido, Japón & Alicata, 1991 \\
\hline 1976 & Rattus norvergicus & Lanistes carinatus & 1996 & Homo sapiens & Egipto* & Archer et al. 2011 \\
\hline 1977 & Rattus norvergicus & $\begin{array}{l}\text { Lissachatina fulica } \\
\text { Bradybaena similaris } \\
\text { Rumina decollata } \\
\text { Subulina octona } \\
\text { Succinea } \mathrm{sp} . \\
\text { Veronicella cubensis } \\
\text { Pomacea } \mathrm{sp} \\
\end{array}$ & 1981 & Homo sapiens & Cuba* & Aguiar et al. 1981 \\
\hline 1978 & Rattus exulans & Lissachatina fulica & 1978 & Homo sapiens & Indonesia* & Alicata, 1991 \\
\hline 1980 & $\begin{array}{l}\text { Sigmodon hispidus } \\
\text { Rattus rattus }\end{array}$ & Vaginula plebeius & 1980 & Homo sapiens & Costa Rica* & Nuñez \& Mirambell, 1981 \\
\hline 1980 & & & 1980 & Homo sapiens & Costa de Marfil* & Nozais et al.1980 \\
\hline 1980 & $\begin{array}{l}\text { Rattus norvergicus } \\
\text { Rattus rattus }\end{array}$ & $\underline{\text { Lissachatina fulica }}$ & 1980 & Homo sapiens & Nueva Zelanda* & Cross, 2007 \\
\hline
\end{tabular}




\begin{tabular}{|c|c|c|c|c|c|c|}
\hline 1984 & $\begin{array}{l}\text { Rattus rattus } \\
\text { Rattus norvegicus }\end{array}$ & $\begin{array}{l}\text { Subulina octona } \\
\text { Aquebana belutina }\end{array}$ & 1986 & Homo sapiens & Puerto Rico* & Anderson et al. 1986 \\
\hline 1984 & $\begin{array}{l}\text { Rattus norvergicus } \\
\text { Rattus rattus }\end{array}$ & $\begin{array}{l}\text { Lissachatina fulica } \\
\text { Pomacea canaliculata }\end{array}$ & 1984 & Homo sapiens & Hong Kong, China & Alicata, 1991 \\
\hline 1984 & $\begin{array}{l}\text { Rattus norvergicus } \\
\text { Rattus rattus }\end{array}$ & $\underline{\text { Lissachatina fulica }}$ & 1984 & $\begin{array}{l}\text { Sapos/Ranas }{ }^{1} \\
\text { Homo sapiens }\end{array}$ & Nueva Caledonia* & Alicata, 1991 \\
\hline 1984 & $\begin{array}{l}\text { Rattus norvergicus } \\
\text { Rattus rattus }\end{array}$ & $\underline{\text { Lissachatina fulica }}$ & 1984 & Homo sapiens & Vanatau* & Alicata, 1991 \\
\hline 1984 & $\begin{array}{l}\text { Rattus norvergicus } \\
\text { Rattus rattus }\end{array}$ & $\underline{\text { Lissachatina fulica }}$ & 1984 & Homo sapiens & Vietnam, China & Alicata, 1991 \\
\hline 1984 & $\begin{array}{l}\text { Rattus norvergicus } \\
\text { Rattus rattus }\end{array}$ & $\underline{\text { Lissachatina fulica }}$ & 1984 & Homo sapiens & Samoa* & Alicata, 1991 \\
\hline 1984 & $\begin{array}{l}\text { Rattus rattus } \\
\text { Rattus exulans }\end{array}$ & $\underline{\text { Lissachatina fulica }}$ & 1984 & Homo sapiens & Fiji* & Alicata, 1991 \\
\hline 1984 & $\begin{array}{l}\text { Rattus norvergicus } \\
\text { Rattus rattus }\end{array}$ & Lissachatina fulica & 1984 & Homo sapiens & Nueva Guinea* & Alicata, 1991 \\
\hline 1987 & $\begin{array}{l}\text { Rattus rattus } \\
\text { Rattus norvegicus }\end{array}$ & Subulina octona & 1990 & Hylobates lyr & Nassau, Bahamas & Vargas et al. 1992 \\
\hline 1988 & $\begin{array}{l}\text { Rattus norvergicus } \\
\text { Podomys floridanus } \\
\text { Didelphis virginiana } \\
\text { Varecia variegata }\end{array}$ & Moluscos no identificados & 1992 & $\begin{array}{l}\text { Alouatta caraya } \\
\text { Lemur variegatus } \\
\text { Cercophitecus talapon } \\
\text { Homo sapiens }\end{array}$ & Luisiana, USA & $\begin{array}{l}\text { Capmbell \& Little } 1988 \\
\text { Cross et al. } 2004\end{array}$ \\
\hline 1992 & Rattus rattus & Subulina octona & 1992 & Homo sapiens & Republica Dominicana* & Vargas et al. 1992 \\
\hline 1992 & $\begin{array}{l}\text { Rattus norvergicus } \\
\text { Rattus rattus }\end{array}$ & $\underline{\text { Lissachatina fulica }}$ & 1992 & $\begin{array}{l}\text { Lagartijas }{ }^{1} \\
\text { Homo sapiens }\end{array}$ & India* & Cross, 2007 \\
\hline 1992 & $\begin{array}{l}\text { Rattus norvergicus } \\
\text { Rattus rattus }\end{array}$ & $\underline{\text { Lissachatina fulica }}$ & 1992 & Homo sapiens & Malasia* & Cross, 2007 \\
\hline 1994 & $\begin{array}{l}\text { Rattus rattus } \\
\text { Rattus norvegicus }\end{array}$ & Thelidomus asper & 1994 & Homo sapiens & Jamaica* & Cross, 2004 \\
\hline 1995 & $\begin{array}{l}\text { Rattus norvergicus } \\
\text { Rattus rattus }\end{array}$ & $\begin{array}{l}\text { Lissachatina fulica } \\
\text { Pomacea canaliculata } \\
\text { Pila } s p\end{array}$ & 1995 & $\begin{array}{l}\text { Lagartijas }^{1} \\
\text { Homo sapiens }\end{array}$ & Tailandia* & Cross, 2007 \\
\hline 1995 & $\begin{array}{l}\text { Rattus norvergicus } \\
\text { Rattus rattus }\end{array}$ & $\underline{\text { Lissachatina fulica }}$ & 1995 & $\begin{array}{l}\text { Lagartijas }{ }^{1} \\
\text { Homo sapiens }\end{array}$ & Sry Lanka* & Cross, 2007 \\
\hline 2002 & $\begin{array}{l}\text { Rattus rattus } \\
\text { Rattus norvergicus }\end{array}$ & Subulina octona & & & Puerto Principe, Haiti & Raccurt et al. 2003 \\
\hline
\end{tabular}




\begin{tabular}{|c|c|c|c|c|c|c|}
\hline 2003 & $\begin{array}{l}\text { Rattus rattus } \\
\text { Rattus norvergicus }\end{array}$ & $\begin{array}{l}\text { Lissachatina fulica } \\
\text { Zachrysia provisoria } \\
\text { Bradybaena similaris } \\
\text { Alcadia striata }\end{array}$ & 2003 & Hylobates lyr & Florida, USA & $\begin{array}{l}\text { Cross et al. } 2004 \\
\text { Duffy et al. } 2004\end{array}$ \\
\hline 2004 & & & 2004 & & Nigeria** & Ping Wang et al. 2008 \\
\hline 2006 & & & 2006 & & Alemania** & Ping Wang et al. 2008 \\
\hline 2007 & $\begin{array}{l}\text { Rattus rattus } \\
\text { Rattus norvegicus }\end{array}$ & $\begin{array}{l}\text { Lissachatina fulica } \\
\text { Bradybaena silimaris } \\
\text { Saranisula marginata } \\
\text { Subulina octona }\end{array}$ & 2007 & Homo sapiens & Espiritu Santo, Brasil & Caldeira et al. 2007 \\
\hline 2008 & Rattus norvergicus & $\begin{array}{l}\text { Lissachatina fulica } \\
\text { Pomacea } \text { sp. }\end{array}$ & 2008 & Homo sapiens & Guayas, Ecuador & $\begin{array}{l}\text { Martini Robles \& Dorta } \\
\text { Contreras, } 2016\end{array}$ \\
\hline \multirow[t]{2}{*}{2008} & & & 2008 & & Bélgica** $^{*}$ & Ping Wang et al. 2008 \\
\hline & & $\begin{array}{l}\text { Lissachatina fulica } \\
\text { Subulina octona } \\
\text { Pomacea lineata }\end{array}$ & 2009 & Homo sapiens & Pernambuco, Brasil & Thiengo et al. 2010 \\
\hline \multirow[t]{2}{*}{2010} & Rattus norvergicus & $\begin{array}{l}\text { Lissachatina fulica } \\
\text { Saranisula marginata }\end{array}$ & 2007 & Homo sapiens & Rio de Janeiro, Brasil & $\begin{array}{l}\text { Simoes et al. } 2011 \\
\text { Morasutti et al. } 2014\end{array}$ \\
\hline & & $\begin{array}{l}\text { Lissachatina fulica } \\
\text { Bradybaena silimaris } \\
\text { Saranisula linguaeformis } \\
\text { Saranisula marginata } \\
\text { Subulina octona }\end{array}$ & 2010 & Homo sapiens & São Paulo, Brasil & Espirito Santo et al. 2013 \\
\hline 2010 & $\begin{array}{l}\text { Rattus rattus } \\
\text { Rattus norvegicus }\end{array}$ & $\begin{array}{l}\text { Lissachatina fulica } \\
\text { Bradybaena silimaris }\end{array}$ & 2010 & Homo sapiens & Santa Catarina, Brasil & Ohlweiler et al. 2010 \\
\hline 2010 & Rattus rattus & & & & Tenerife, España & Foronda et al. 2010 \\
\hline 2013 & $\begin{array}{l}\text { Rattus rattus } \\
\text { Rattus norvegicus }\end{array}$ & $\begin{array}{l}\text { Lissachatina fulica } \\
\text { Saranisula marginata } \\
\text { Subulina octona }\end{array}$ & & & Pará, Brasil & Morasutti et al. 2014 \\
\hline 2013 & Rattus norvergicus & $\begin{array}{l}\text { Lissachatina fulica } \\
\text { Bradybaena silimaris }\end{array}$ & & Homo sapiens & Rio Grande do Sul, Brasil & $\begin{array}{l}\text { Cognato et al. } 2013 \\
\text { Morasutti et al. } 2014\end{array}$ \\
\hline 2013 & $\begin{array}{l}\text { Rattus rattus } \\
\text { Rattus norvegicus }\end{array}$ & & & Homo sapiens & Guadalupe* & Celine Dard et al. 2017 \\
\hline
\end{tabular}

Sapos/Ranas/Peces/Lagartijas ${ }^{1}$ : Hospedadores paraténicos

*: La capital del país fue citada, cuando la localidad exacta no fue obtenida.

**: Enfermedad que contrajeron viajeros al viajar a países con registros de meningoencefalitis eosinofílica. 
ANEXO III. Registros de enfermedad, hospedadores definitivos e intermediarios de Angiostrongylus costaricensis.

\begin{tabular}{|c|c|c|c|c|c|c|}
\hline $\begin{array}{c}\text { Primer } \\
\text { hallazgo del } \\
\text { parásito adulto }\end{array}$ & Hospedador definitivo & Hospedador intermediario & $\begin{array}{l}\text { Primer registro } \\
\text { de enfermedad }\end{array}$ & Hospedador accidental & Sitio geográfico & Literatura consultada \\
\hline 1971 & $\begin{array}{l}\text { Rattus norvergicus } \\
\text { Sigmodon hispidus } \\
\text { Rattus rattus } \\
\text { Nasua narica } \\
\text { Canis familiaris } \\
\end{array}$ & Saranisula plebeius & 1971 & Homo sapiens & Costa Rica* & $\begin{array}{l}\text { Morera \& Céspedes, } 1971 \\
\text { Monge et al. } 1978 \\
\text { Alfaro-Alarcón et al. } 2015\end{array}$ \\
\hline 1971 & $\begin{array}{l}\text { Sigmodon hispidus } \\
\text { Didelphis virginiana } \\
\text { Procyon lotor } \\
\text { Symphalangus syndactylus }\end{array}$ & & 1971 & Aotus nancymaae & Florida, USA & Uberlaker \& Hall, 1979 \\
\hline \multirow[t]{4}{*}{1973} & $\begin{array}{l}\text { Sigmodon hispidus } \\
\text { Rattus rattus } \\
\text { Liomys adspersus } \\
\text { Oligoryzomys fulvescens } \\
\text { Zygodontomys revicaudas }\end{array}$ & Saranisula plebeius & 1973 & Homo sapiens & Panamá* & Kaminsky, 1996 \\
\hline & & & 1975 & Homo sapiens & São Paulo, Brasil & Zilliota et al. 1975 \\
\hline & & & 1980 & Homo sapiens & Brasília, Brasil & Agostini et al. 1984 \\
\hline & & & 1982 & Homo sapiens & Paraná, Brasil & Ayala, 1987 \\
\hline 1979 & Sigmodon hispidus & & 1994 & Homo sapiens & Texas, USA & Miller et al. 2006 \\
\hline 1981 & $\begin{array}{l}\text { Sigmodon hispidus } \\
\text { Melanomys caliginosus }\end{array}$ & Veronicella occidentalis & 1981 & Homo sapiens & Colombia* & Malek, 1981 \\
\hline \multirow[t]{2}{*}{1982} & Saguinus mystax & & 1982 & & Peru* & Sly et al. 1982 \\
\hline & & & 1982 & Homo sapiens & Tucumán, Argentina & Demo \& Pessat, 1986 \\
\hline 1983 & $\begin{array}{l}\text { Rattus rattus } \\
\text { Rattus norvergicus }\end{array}$ & Veronicella occidentalis & 1983 & Homo sapiens & Ecuador* & Kaminsky, 1996 \\
\hline 1985 & Proechimys sp. & Veronicella occidentalis & 1985 & Homo sapiens & Venezuela* & Kaminsky, 1996 \\
\hline 1990 & $\begin{array}{l}\text { Oligoryzomys nigripes } \\
\text { Sooretamys angouya }\end{array}$ & $\begin{array}{l}\text { Belocaulis angustipes } \\
\text { Bradybaena similaris } \\
\text { Limacus flavus }\end{array}$ & 1983 & Homo sapiens & Rio Grande do Sul, Brasil & $\begin{array}{l}\text { Agostini et al. } 1984 \\
\text { Graeff Texeira et al. } 1990\end{array}$ \\
\hline
\end{tabular}




\begin{tabular}{|c|c|c|c|c|c|c|}
\hline & & $\begin{array}{l}\text { Phyllocaulis soleiformis } \\
\text { Phyllocaulis variegatus }\end{array}$ & & & & \\
\hline & & $\begin{array}{l}\text { Phyllocaulis variegatus } \\
\text { Saranisula linguaeformis } \\
\text { Deroceras laeve }\end{array}$ & 1987 & Homo sapiens & Santa Catarina, Brasil & Ayala, 1987 \\
\hline & & & 1991 & Homo sapiens & Minas Gerais, Brasil & Rocha et al. 1991 \\
\hline 1991 & Sigmodon hispidus & $\begin{array}{l}\text { Saranisula plebeius } \\
\text { Veronicella occidentalis }\end{array}$ & 1991 & Homo sapiens & Nicaragua* & Duarte et al. 1991 \\
\hline 1992 & Sigmodon hispidus & $\begin{array}{l}\text { Saranisula plebeius } \\
\text { Veronicella occidentalis }\end{array}$ & 1992 & Homo sapiens & El Salvador* & Kaminsky, 1996 \\
\hline 1992 & $\begin{array}{l}\text { Rattus rattus } \\
\text { Rattus norvegicus }\end{array}$ & & 1992 & Homo sapiens & Isla de Guadalupe* & Kaminsky, 1996 \\
\hline 1992 & $\begin{array}{l}\text { Rattus rattus } \\
\text { Rattus norvergicus }\end{array}$ & & 1992 & Homo sapiens & Republica Dominicana* & Maldonado Jr et al. 2012 \\
\hline 1992 & $\begin{array}{l}\text { Rattus rattus } \\
\text { Rattus norvergicus }\end{array}$ & & 1992 & Homo sapiens & Puerto Rico* & Maldonado Jr et al. 2012 \\
\hline \multirow[t]{2}{*}{1994} & Sigmodon hispidus & $\begin{array}{l}\text { Saranisula plebeius } \\
\text { Veronicella occidentalis }\end{array}$ & 1994 & Homo sapiens & Guatemala* & Kaminsky, 1996 \\
\hline & & & 1995 & Homo sapiens & Espiritu Santo, Brasil & Pena et al. 1995 \\
\hline 1996 & $\begin{array}{l}\text { Sigmodon hispidus } \\
\text { Peromyscus spp. } \\
\text { Mus musculus }\end{array}$ & $\begin{array}{l}\text { Saranisula plebeius } \\
\text { Veronicella occidentalis }\end{array}$ & 1972 & Homo sapiens & $\begin{array}{l}\text { Valle de Yegüare, } \\
\text { Honduras }\end{array}$ & $\begin{array}{l}\text { Zuñiga et al. } 1983 \\
\text { Kaminsky } 1996\end{array}$ \\
\hline 2008 & Akodon montensis & & & & Misiones, Argentina & Robles et al. 2016 \\
\hline
\end{tabular}

*: La capital del país fue citada, cuando la localidad exacta no fue obtenida. 
Anexo IV. Medias bimestrales de la las variables ambientales.

\begin{tabular}{|c|c|c|c|c|c|}
\hline Bimestre & $\begin{array}{c}\text { Precipitaciones } \\
\text { media } \\
\text { bimestral }\end{array}$ & $\begin{array}{c}\text { Temperatura } \\
\text { máxima } \\
\text { media } \\
\text { bimestral }\end{array}$ & $\begin{array}{c}\text { Temperatura } \\
\text { mínima } \\
\text { media } \\
\text { bimestral }\end{array}$ & $\begin{array}{c}\text { Humedad } \\
\text { máxima } \\
\text { media } \\
\text { bimestral }\end{array}$ & $\begin{array}{c}\text { Humedad } \\
\text { mínima } \\
\text { media } \\
\text { bimestral }\end{array}$ \\
\hline $\begin{array}{c}\text { Oct-Nov } \\
\mathbf{2 0 1 3}\end{array}$ & 15,8 & 29,3 & 17,5 & 94,3 & 60,1 \\
\hline $\begin{array}{c}\text { Feb-mar } \\
\text { 2014 }\end{array}$ & 5,61 & 31,69 & 19,61 & 94,76 & 59,05 \\
\hline $\begin{array}{c}\text { Jun-Jul } \\
\mathbf{2 0 1 4}\end{array}$ & 9,13 & 21,92 & 12,5 & 99,63 & 75,19 \\
\hline $\begin{array}{c}\text { Oct-Nov } \\
\mathbf{2 0 1 4}\end{array}$ & 4,16 & 31,13 & 18,5 & 94,3 & 54,05 \\
\hline $\begin{array}{c}\text { Feb-mar } \\
\mathbf{2 0 1 5}\end{array}$ & 8,5 & 31 & 20,3 & 98,67 & 61,22 \\
\hline $\begin{array}{c}\text { Jun-Jul } \\
\text { 2015 }\end{array}$ & 5,23 & 24,21 & 13,38 & 99,83 & 65 \\
\hline $\begin{array}{c}\text { Oct-Nov } \\
\text { 2015 }\end{array}$ & 9,95 & 27,87 & 19,26 & 99 & 70 \\
\hline
\end{tabular}


ANEXO V. Puntos de presencia de Lissachatina fulica en Sudamérica.

\begin{tabular}{|c|c|c|}
\hline ID & Sitio Geográfico & Literatura consultada \\
\hline 1 & Bahia, Brasil & Thiengo et al. 2007 \\
\hline 2 & Espírito Santo; Brasil & Thiengo et al. 2007 \\
\hline 3 & Goiás, Brasil & Thiengo et al. 2007 \\
\hline 4 & Minas Gerais, Brasil & Thiengo et al. 2007 \\
\hline 5 & Belo Horizonte, Brasil & Thiengo et al. 2007 \\
\hline 6 & Paraná, Brasil & Thiengo et al. 2007 \\
\hline 7 & Pernambuco, Brasil & Thiengo et al. 2007 \\
\hline 8 & Rio de Janeiro; Brasil & Thiengo et al. 2007 \\
\hline 9 & Santa Catarina, Brasil & Thiengo et al. 2007 \\
\hline 10 & São Paulo, Brasil & Thiengo et al. 2007 \\
\hline 11 & Puerto Iguazú, Misiones & Gutiérrez Gregoric et al. 2011 \\
\hline 12 & Corrientes Capital, Corrientes & Gutiérrez Gregoric et al. 2013 \\
\hline 13 & Puerto Suarez, Bolivia & Correoso \& Coelho, 2009 \\
\hline 14 & Perú & Correoso \& Coelho, 2009 \\
\hline 15 & Provincia de Misiones, Paraguay & Ciomperlik et al. 2013 \\
\hline 16 & Dep de Amazonas, Colombia & Linares et al. 2013 \\
\hline 17 & Dep. de Cauca, Colombia & Linares et al. 2013 \\
\hline 18 & Dep. de Cauca, Colombia & Linares et al. 2013 \\
\hline 19 & Dep. de Caqueta, Colombia & Linares et al. 2013 \\
\hline 20 & Dep. de Sucre, Colombia & Linares et al. 2013 \\
\hline 21 & Dep. de Boyaca, Colombia & Linares et al. 2013 \\
\hline 22 & Dep. de Casanare, Colombia & Linares et al. 2013 \\
\hline 23 & Dep. de Tolima, Colombia & Linares et al. 2013 \\
\hline 24 & Dep. de Huila, Colombia & Linares et al. 2013 \\
\hline 25 & Dep. de Santander, Colombia & Linares et al. 2013 \\
\hline 26 & Dep. de Meta, Colombia & Linares et al. 2013 \\
\hline 27 & Dep. de Nariño, Colombia & Linares et al. 2013 \\
\hline 28 & Prov. de Galápagos, Ecuador & Miquel \& Herrera, 2014 \\
\hline 29 & Prov. de Galápagos, Ecuador & Miquel \& Herrera, 2014 \\
\hline 30 & Prov. de Manabí, Ecuador & Correoso \& Coelho, 2009 \\
\hline 31 & Prov. de Chimborazo, Ecuador & Correoso \& Coelho, 2009 \\
\hline 32 & Prov. de Cotopaxi, Ecuador & Correoso \& Coelho, 2009 \\
\hline 33 & Prov. de Cañar, Ecuador & Correoso \& Coelho, 2009 \\
\hline 34 & Prov. de Pichincha, Ecuador & Correoso \& Coelho, 2009 \\
\hline 35 & Prov. de Esmeraldas, Ecuador & Correoso \& Coelho, 2009 \\
\hline 36 & Caracas, Venezuela & Sistema Venezolano de información sobre \\
\hline 37 & Guanare, Venezuela & la diversidad biológica, 2012 \\
\hline 38 & Delta Amacuro, Venezuela & Sistema Venezolano de información sobre \\
\hline 39 & Apure, Venezuela & la diversidad biológica, 2012 \\
\hline
\end{tabular}




\section{BIBLIOGRAFÍA}

Acha, P., Szyfres, B. 2003. Angiostrongyliasis, intestinal angiostrongyliasis, angiostrongyliasis (Parastrongylus cantonensis). Zoonoses and Communicable Diseases Man and Animals Volumen 3. Pan American Health Organization, Scientific and Tech Publications, Washington, DC: 225-231 pp.

Agostini, A.A., Marcolan, A.M., Lisot, J.M., Lisot, J.U. 1984. Angiostrongilíase abdominal, estudo anátomo patológico de quatro casos observados no Rio Grande do Sul, Brasil. Mem Inst Oswaldo Cruz., 79: 443-445.

Aguiar, P.H., Morera, J., Pascual, J. 1981. First record of Angiostrongylus cantonensis in Cuba. Am J Trop Med Hyg., 30: 963-965.

Albuquerque, F.S., Peso-Aguiar, M.C., Albuquerque, M.J.T, Gálvez, L. 2007. Do climate variables and human density affect Achatina fulica (Bowdich) (Gastropoda: Pulmonata) shell length, total weight and condition factor? Braz J Biol., 69: 879-885.

Alfaro-Alarcón, A., Veneziano, V., Galiero, G., Cerrone, A., Gutierrez, N., Chinchilla, A., Annoscia, G., Colella, V., Dantas-Torres, F., Otranto, D., Santoro, M. 2015. First report of a naturally patent infection of Angiostrongylus costaricensis in a dog. Vet Parasitol., 212: 431-434.

Alicata, J.E. 1991. The discovery of Angiostrongylus cantonensis as a cause of human eosinophilic meningitis. Parasitol Today., 7: 151-153.

Anderson, R.M., May, R.M. 1979. Population biology of infectious diseases: Part I. Nature, 280: 361-367.

Anderson, R.C., Chabaud, A.G., Wilmott, S. 2010. Keys to the Nematode Parasite of Vertebrates. Archival Volume. CABI International, Wallingford, UK: 200 pp.

Archer, C.E., Appleton, C.C., Mukaratirwa, S., Hope, K.J. 2011. The rat lung-worm Angiostrongylus cantonensis: A first report in South Africa. S Afr Med J., 101: 174-175.

Ash, L. 1970. Diagnostic morphology of the third stage larvae of Angiostrongylus cantonensis, Angiostrongylus vasorum, Aelurostrongylus abstrusus, and Anafi laroides rostratus (Nematoda: Metastrongyloidea). J Parasitol., 56: 249-253.

Ayala, M.A. 1987. Angiostrongiloidíase abdominal: seis casos observados no Paraná e em Santa Catarina, Brasil. Mem Inst Oswaldo Cruz., 82: 29-36.

Barger, M. A., Hnida, J.A. 2008. Survey of trematodes from terrestrial gastropods and small mammals in southeastern Nebraska. Comp Parasitol., 75: 308-314. 
Barreto-Lima, A.F., Alves dos Anjos, L. 2014. Occurrence of Strongyluris oscari (Nematoda; Heterakidae) in Enyalius bilineatus (Squamata: Leiosaurinae) from the Brazilian Atlantic Forest. Herpetol Notes., 7: 455-456.

Barutzki, D., Schaper, R. 2013. Occurrence and regional distribution of Aelurostrongylus abstrusus in cats in Germany. Parasitol Res., 112: 855-861.

Bellard, C., Bertelsmeier, C., Leadley, P., Thuiller, W., Courchamp, F. 2012. Impacts of climate change on the future of biodiversity. Ecol Lett., 15: 365-377.

Borda, E., Rea, M.J., Mosqueda, L., Benitez, O. 2006. Hospedadores intermediarios y definitivos de Schistosoma mansoni en la provincia de Corrientes, Argentina. Comunicaciones Científicas y Tecnológicas. Universidad Nacional del Nordeste. Resumen M-127.

Borrero, F.J., Breure, A.S., Christensen, C., Correoso, M., Mogollón Ávila, V. 2009. Into the Andes: Three new introductions of Lissachatina fulica (Gastropoda, Achatinidae) and its potential distribution in South America. Tentacle., 17: 6-8.

Bush, A.O., Lafferty, K.D., Lotz, J.M., Shostakll, A.W. 1997. Parasitology meets ecology on its own terms: Margolis et al. revisited. J Parasitol., 83: 575-583.

Bush, A.O., Fernández, J.C., Esch, G.W., Seed, J.R. 2011. Parasitism: The Diversity and Ecology of Animal Parasites. Cambridge University Press, Cambridge, U.K: 566 p.

Butcher, A.R., Groove, D.I. 2001. Description of the life-cycle stages of Brachylaima cribbi n. sp. (Digenea: Brachylaimidae) derived from eggs recovered from human feces in Australia. Syst Parasitol., 49: 211-221.

Caldeira, R.L., Mendonça, C.L., Goveia, C.O., Lenzi, H.L., Graeff-Teixeira, C., Lima, W.S., Mota, E.M., Pecora, I.L., Medeiros, A.M., Carvalho, O.S. 2007. First record of molluscs naturally infected with Angiostrongylus cantonensis (Chen, 1935) (Nematoda: Metastrongylidae) in Brazil. Mem Inst Oswaldo Cruz., 102: 887-889.

Campbell, B.G., Little, M.D. 1988. The finding of Angiostrongylus cantonesis in rats in New Orleans. Am J Trop Med Hyg.; 38: 568-573.

Cardillo, N., Clemente, A., Pasqualetti, M., Borrás, P., Rosa, A., Ribicich, M. 2014.

First report of Aelurostrongylus abstrusus in domestic land snail Rumina decollata, in the Autonomous city of Buenos Aires. InVet., 16: 15-22.

Carvalho da Silva, E., Omena, E.P. 2014. Population dynamics and reproductive biology of Achatina fulica Bowdich, 1822 (Mollusca, Gastropoda) in Salvador Bahia. Biota Neotrop., 14: 1-11.

Carvalho, O.S., Teles, H.M.S., Mota, E.M., Mendonca C.L., Lenzi, H.L. 2003. Potentiality of Achatina fulica Bowdich, 1822 (Mollusca: Gastropoda) as 
intermediate host of the Angiostrongylus costaricensis Morera \& Céspedes 1971. Rev Soc Bras Med Trop., 36: 743-745.

Céspedes, R., Salas, J., Mekbel, S., Troper, L., Müllner, F., Morera, P. 1967. Granulomas entéricos y linfáticos con intensa eosinofilia tisular producida por un estrongilídeo (Strongylata). Acta Med Costa Rica., 10: 235-255.

Cognato, B.B., Morassutti, A.L., da Silva, A.A., Graeff-Teixeira, C. 2013. First report of Angiostrongylus cantonensis in Porto Alegre, Rio Grande does Sul, southern Brazil. Rev Soc Bras Med Trop., 46: 664-665.

Correoso Rodríguez, M. 2006. Estrategia preliminar para evaluar y erradicar Achatina fulica (Gastropoda: Achatinaceae) en Ecuador. Boletín Técnico IASA, Serie Zoológica., 2: $45-52$.

Correoso Rodríguez, M., Coello, M. 2009. Modelación y distribución de Lissachatina fulica (Gastropoda: Achatinidae) en Ecuador. Potenciales impactos ambientales y sanitarios. Rev Geoespacial., 6: 79-90.

Cross, J. 2004. Angiostrongylus (Parastrongylus) cantonensis in the western hemisphere. Southeast Asian J Trop Med Public Health., 35: 107-111.

Cross, J. 2007. The spread of Angiostrongyliasis: The globetrotting rat lungworm. Southeast Asian J Trop Med Public Health., 38: 43-46.

Demo, O.J., Pessat, A.O. 1986. Angiostrongyliasis abdominal. Primer caso humano encontrado en la Argentina. Prensa Med Arg., 73: 732-738.

Diaz, J.I., Lorenti E., Valente, R., Capasso, S., Gutiérrez Gregoric, D.E, Navone, G.T. Estado actual del conocimiento de las parasitosis del molusco invasor Achatina fulica en la Argentina su importancia como posible vector de angiostrongiliasis. 2013. Moluscos de interés sanitario en la Argentina. Instituto Nacional de Medicina Tropical-Msal, Puerto Iguazú: 40-53 pp.

Di Bitetti, M.S., Placci, G., Dietz, L.A. 2003. A biodiversity vision for the Upper Paraná Atlantic Forest ecoregion. Washington, D.C. USA: 154 p.

Dorta Contreras, A. J., Núñez Fernández, F.A., Pérez Martín, O., Lastre González, M., Magraner Tarrau, M.E., Bu Coifiu Fanego, R., Noris García, E., Interián Morales, M.T., Martínez Delgado, J.F., Sánchez Zulueta, E. 2007. Peculiaridades de la meningoencefalitis por Angiostrongylus cantonensis en América. Rev Neurol., 45: $755-763$.

Duarte, Z., Moreira, P., Vuong, P. 1991. Abdominal angiostrongyliasis in Nicaragua: a clinico-pathological study on a series of twelve case reports. Ann Parasitol Hum Comp., 66: 259-262. 
Duarte, F.H., Vieira, F.M., Louzada, G.L., Bessa, E.C.A., Souzalima, S. 2007. Occurrence Angiostrongylus vasorum (Baillet, 1866) (Nematoda, Angiostrongylidae) in Cerdocyon thous Linnaeus, 1766 (Carnivora, Canidae) in Minas Gerais State Brazil. Arq Bras Med Vet Zootec., 59: 1086-1088.

Duffy, M.S., Miller, C.L., Kinsella, J.M,, Lahunta, A. 2004. Parastrongylus cantonensis in a nonhuman primate, Florida. Emerg Infect Dis., 10: 2207-2210.

Esch, G.W., Barger, M.A., Fellis K.J. 2002. The Transmission of Digenetic Trematodes: Style, Elegance, Complexity. Integ. and Comp. Biol., 42:304-312.

Espírito Santo, M.C., Pinto, P.L., da Mota, D.J., Gryschek, R.C. 2013. The first case of Angiostrongylus cantonensis eosinophilic meningitis diagnosed in the city of São Paulo, Brazil. Rev Inst Med Trop Sao Paulo., 55: 129-132.

Fischer, M.L., Simião, M.S., Colley, E., Costa, L.C. M.; Rúbio, G. 2005. Panorama do caramujo gigante africano Achatina fulica Bowdich, 1822 no Estado do Paraná: o provável ponto de entrada da espécie invasora no Brasil. I Simpósio Brasileiro sobre espécies exóticas invasoras, Brasília.

Fischer, M.L., Costa, L.C.M. 2010. O caramujo Gigante Africano Achatina fulica no Brasil. Universitaria Champagnat, Curitiba, Brasil: 13-99 pp.

Foronda, P., López-Gonzáleza, M., Miquel, J., Torres, J., Segovia, M., Abreu-Acosta, N., Casanova, J.C., Valladares, B., Mas-Coma, S., Bargues, M.D., Feliu, C. 2010. Finding of Parastrongylus cantonensis (Chen, 1935) in Rattus rattus in Tenerife, Canary Islands (Spain). Acta Trop., 114: 123-127.

Franco-Acuña, D.O., Pinheiro, J., Torres, E.J.L, Lanfredi, R.M., Brandolini, S.V. 2009. Nematode cysts and larvae found in Achatina fulica Bowdich, 1822. J Invertebr Pathol., 100: 106-110.

Gibson, D.I., Jones, A., Bray, R.A. 2001. Key to the trematoda (Vol. 1) Wallingford: CABI Publishing.

Graeff-Teixeira, C., Ávila-Pires, F.D., Machado, R.C.C, Camillo-Coura, L., Lenzi, H.L. 1990. Identificação de roedores silvestres como hospedeiros do Angiostrongylus costaricensis no sul do Brasil. Rev Inst Med Trop São Paulo., 32: 147-150.

Gutiérrez Gregoric, D.E., Nuñez, V., Vogler, R.E., Rumi, A. 2011. Invasion of the Argentinean Paranense rainforest by giant African snail, Achatina fulica. Am Malacol Bull., 29: 135-137.

Gutiérrez Gregoric, D.E., Beltramino, A., Vogler, R.E., Rumi, A. 2013. Expansión del rango de distribución de Achatina fulica (Gastropoda) y su concordancia con modelos predictivos. Amici Molluscarum., 21: 17-21. 
Haseeb, M.A., Fried, B. 1997. Modes of transmissions of trematode infections and their control. Trematode Biology. Florida, USA: 466 pp.

Hassol, S.J. 2004. Impacts of a warming Arctic, Arctic climate impact assessment. New York, Cambridge University Press, USA.

Jefferies, R., Vrhovec, M.G., Wallner, N., Catalan, D.R. 2010. Aelurostrongylus abstrusus and Troglostrongylus sp. (Nematoda: Metastrongyloidea) infections in cats inhabiting Ibiza, Spain. Vet Parasitol., 173: 344-348.

Kaminsky, R.G., Caballero, R., Andrews, K. 1995. Presencia de Angiostrongylus costaricensis en Honduras y sus relaciones agro-ecológicas y humanas. Parasitología al Día., 19: 81-90.

Kaminsky, R.G. 1996. Situación actual de Angiostrongylus costaricensis y la infección en humanos y animales en las Américas. Rev Med Hondureña; 64: 139-147.

Koch, J., Willesen, J.L. 2009. Canine pulmonary angiostrongylosis: An update. Vet J., 179: $348-359$.

Linares, E., Avendaño, J., Martínez, A. Rojas, A. 2013. El caracol gigante africano, un visitante indeseado. Mini guía de campo Instituto de Ciencias Naturales. Universidad Nacional de Colombia.

Lowe, S., Browne, M., Boudjelas, S., De Poorter, M. 2004. Cien de las especies Exóticas Invasoras más dañinas del mundo. Una selección del Global Invasive Species Database. Nueva Zelanda: 11 pp.

Lunaschi, L.I., Drago, F.B. 2012. Digenean parasites of Cariama cristata (Aves, Gruiformes) from Formosa Province, Argentina, with the description of a new species of the genus Strigea. Acta Parasitol., 57: 26-33.

Maciel-Mata, C.A., Manríquez-Morán, N., Octavio-Aguilar, P., Sánchez-Rojas, G. 2015. El área de distribución de las especies: Revisión del concepto. Acta Universitaria, 25: 3-19.

Mack, R.N., Simberloff, D., Lonsdale, W.M., Evans, H., Clout, M., Bazzaz, F. 2000. Invasiones Biológicas: Causas, Epidemiología, Consecuencias globales y Control. Top Ecol., 5: 1-22.

Maldonado Jr, A, Simões, R., Thiengo, S.C. Angiostrongyliasis in the Americas. 2012. Zoonosis. Tech, Rijeka, 303-320 p.

Marquardt, W., Demaree, R., Grieve, R. 2000. Parasitology, vector and biology. San Diego, California. 
Martin-Alonso, A., Abreu-Yanes, E., Feliu, C., Mas-Coma S, Bargues, M.D., Valladares, B., Foronda, P. 2015. Intermediate hosts of Angiostrongylus cantonensis in Tenerife, Spain. Plos One., 10: 1-10.

Martínez-Escarbassiere, R., Martínez, E.O., Castillo, O. 2008. Distribución geográfica de Achatina (Lissachatina) fulica (Bowdich, 1882) (GastropodaStylommatophora-Achatinidae) en Venezuela. Mem Fund La Salle de Ciencias Naturales., 169: 93-106.

Martínez, F.A. 1986. Helmintofauna de los mamíferos silvestres. Trematodes. Vet Arg., 3: 544-551.

Martini Robles, L., Dorta-Contreras, A.J. 2016. Angiostrongylus cantonensis. Emergencia en América. Ecuador: 1-289 pp.

Matinella, L., Morales, G., Sierra, C., Silva, I., Pino, L. 2009. Achatina fulica: Un caracol de interés para la salud pública. Revista Inia Hoy., 6: 200-210.

May, R.M., Anderson, R.M. 1979. Population biology of infectious diseases: Part II. Nature., 280: 455-461.

Mead, A.R. 1961. The giant African snail; a problem in economic malacology. University of Chicago Press: 257 pp.

Meyer III, W.M., Hayes, K.A., Meyer, A.L. 2008. Giant African snail, Achatina fulica, as a snail predator. Amer Malac Bull., 24: 117-119.

Ministerio de Turismo de Misiones. 2013.

Miquel, S.E., Herrera, H.W. 2014. Catalogue of terrestrial gastropods from Galápagos (except Bulimulidae and Succineidae) with description of a new species of Ambrosiella odhner (Achatinellidae) (Mollusca: Gastropoda). Arch Molluskenkund., 143: 107-133.

Miyazaki, H. 1991. Helminthic Zoonoses. International Foundation of Japan: 347-355 pp.

Monge, E., Arroyo, R., Solano, E. 1978. A new definitive natural host of Angiostrongylus costaricensis (Morera and Céspedes 1971). J Parasitol., 64: 34.

Morassutti, A.L., Thiengo, S.C., Fernandez, M., Sawanyawisuth, K., Graeff-Teixeira, C. 2014. Eosinophilic meningitis caused by Angiostrongylus cantonensis: an emergent disease in Brazil. Mem Inst Oswaldo Cruz., 109: 399-407.

Morera, P. 1967. Granulomas entéricos v linfáticos con intensa eosinofilia tisular producidos por un estrongilideo (Strongylata: Railiet y Henry, 1913). Aspecto parasitológico (Nota Previa). Acta Med Costa Rica., 10: 257-263. 
Morera P, Céspedes R. 1971. Angiostrongylus costaricensis n. sp. (Nematoda: Metastrongyloidea), a new lungworm occurring in man in Costa Rica. Rev Biol Trop., 18: 173-185.

Nozais, J.P., Moreau, J., Morlier, G., Kouame, J., Doucet, J. 1980. Premier cas de méningite à éosinophiles en Côte-d'Ivoire avec présence d'un Parastrongylus sp. dans le liquide céphalorachidien. Bull Soc Pathol Exot Filiales., 73: 179-182.

Núñez, R., Mirambell, F. 1981. Angiostrongilosis abdominal un caso de conducta conservadora. Rev Med Hosp Nac Niños de Costa Rica., 16: 109-116.

Ohlweiler, P.F., Yoshika Takahashi, F., Guimaraes, M., Rodrigues Gomes, S., Kawano, T. 2010. Familia Achatinidae. Manual de Gastropodes limnicos e terrestres do Estado de San Pablo asociados as helmintose. Porto Alegre Brazil Press: 223 pp.

Ortiz, Z., Esandi, M.E., Bortman, M. 2004. Epidemiología básica y vigilancia de la Salud. Ministerio de Salud-Banco Mundial.

Pena, G.P.M, Andrade de Filho, J.S., Assis, S.C. 1995. Angiostrongylus costaricensis: First record of its occurrence in the state of Espiritu Santo, Brazil, and a review of its geographic distribution. Rev Inst Med Trop São Paulo., 37: 369-374.

Phillips, S. J., Anderson, R. P., Schapire, R. E. 2006. Maximum entropy modeling of species geographic distributions. Ecol Modell., 190: 231-259.

Ping Wang, Q., Hua Lai, D., Quan Zhu, X., Guang Chen, X., Rong Lun, Z. 2008 Human Angiostrongyliasis. Lancet Infect. Dis., 8: 621-630.

Poulin R., Morand, S. 2004. Parasite Biodiversity. Washington, USA.

Poulin R. 2007. Evolutionary ecology of parasites. University Press, Princeton.

Prepelitchi, L. 2009. Ecoepidemiología de Fasciola hepatica (Trematoda, Digenea) en el norte de la provincia de Corrientes destacando aspectos ecológicos de Lymnaea columella (Pulmonata, Lymnaeidae) y su rol como hospedador intermediario. Tesis doctoral. Facultad de Ciencias Exactas y Naturales, Universidad de Buenos Aires, Buenos Aires.

Pritchard, M.H., Kruse, G.O. 1982. The collection and preservation of animal parasites. University of Nebraska Press, Nebraska,USA: 141 pp.

Rabinovich, J.E. 1980. Introducción a la ecología de poblaciones animales. Continental S.A, México.

Raccurt, C., Blaise, J., Durette-Desset, M. 2003. Présence d'Angiostrongylus cantonensis en Haiti. Trop Med Int Health., 8: 423-426. 
Raut, S.K., Barker, G.M. 2002. Achatina fulica Bowdich and other Achatinidae as pests in tropical agriculture. Molluscs as crop pests. CABI Publishing, Wallingford: 55-114 pp.

Robles, M.R., Kinsella, J.M., Galliari, C., Navone, G.T. 2016. New host, geographic records, and histopathologic studies of Angiostrongylus spp (Nematoda: Angiostrongylidae) in rodents from Argentina with updated summary of records from rodent hosts and host specificity assessment. Mem Inst Oswaldo Cruz., 111: $181-191$.

Rocha, A., Sobrinho, J.M., Salomão, E.C. 1991. Angiostrongilíase abdominal. Primeiro relato de caso autóctone de Minas Gerais. Rev Soc Bras Med Trop.; 24: 265-268.

Rodrigues Gomes, S. 2007. Filogenia morfológica de Veronicellidae, filogenia molecular de Phyllocaulis Colosi e descrição de uma nova espécie para a família (Mollusca, Gastropoda, Pulmonata). Tesis doctoral. Universidade Federal do Rio Grande do Sul, Rio Grande do Sul.

Rumi, A., Gutiérrez Gregoric, D.E., Núñez, V., Darrigran, G. 2008. Moluscos de agua dulce de Argentina. Rev Biol Trop., 56: 77-111.

Sabina Molina, D., Espinosa Brito, A., Nieto Cabrera, R., Chávez Troya, O., Romero Cabrera, A., Díaz Torralbas, A. 2009. Brote epidémico de meningoencefalitis eosinofílica en una comunidad rural. Rev Cubana Med Trop., 61: 75-81.

Santa Cruz, A.C. 2006. Ecto y endoparásitos de Didelphis alvibentris Temmnick, del NEA (Marsupialia, Didelphidae). Tesis doctoral. Facultad de Ciencias Veterinarias, Universidad Nacional del Nordeste. Corrientes.

Santin, R.A., Miquel, S.E. 2015. Veronicellidae in Argentina: taxonomy, morphology and distribution. Arch Molluskenkund., 144: 105-123.

Sarma, R. R., Munsi, M., Ananthram, A. N. 2015. Effect of climate change on invasion risk of giant African snail (Achatina fulica Férussac, 1821: Achatinidae) in India. PloS One.,10: 1-16.

Scheldeman, X., van Zonneveld, M. 2011. Manual de Capacitación en Análisis Espacial de Diversidad y Distribución de Plantas. Bioversity International, Roma, Italia. $186 \mathrm{pp}$.

Silva Lucas, A.C. 2013. Susceptibilidade e comportamento de Achatina fulica infectada com Angiostrongylus vasorum. Tesina de grado en Universidade Federal de Minas Gerais.

Simões, R.O., Souza, J.G.R., Maldonado Jr, A., Luque, J.L. 2011. Variation in the helminth community structure of three sympatric sigmodontine rodents from the coastal Atlantic Forest of Rio de Janeiro, Brazil. J Helminthol., 85: 171-178. 
Sistema Venezolano de información sobre la diversidad biológica. El caracol gigante africano Achatina fulica: una especie exótica invasora. 2012. Ministerio del poder popular para el ambiente de Venezuela.

Skelley, P.E., Dixon, W.N., Hodges, G. 2010. Giant african land snail and giant South American snails: Field recognition. Florida Department of Agriculture and Consumer Services. Gainesville, Florida.

Sly, D.L., Toft, J.D., Gardiner, C.H., London, W.T. 1982. Spontaneous occurrence of Angiostrongylus costaricensis in marmosets (Saguinus mystax). Lab Anim Sci., 32: $286-289$.

Smith, K.F., Dobson, A.P., McKenzie, F.E., Real, L.A., Smith, D.L., Wilson, M.L. 2005. Ecological theory to enhance infectious disease control and public health policy. Front Ecol Environ., 3: 29-37.

Spratt, D.M. 2015. Species of Angiostrongylus (Nematoda: Metastrongyloidea) in wildlife: a review. Int J Parasitol Parasites. Wildl., 4: 178-189.

Srivastava, P.D. 1992. Problems of Land Snail Pests in Agriculture: A Study of the Giant African Snail. Concept Publishing Company, New Delhi, India.

Teles, H.M.S., Fontes, L.R. 2002 Implicação és da introdução e dispersão de Achatina fulica Bowdich, 1822 no Brasil. Boletim Inst Adolfo Lutz., 12: 3-5.

Thiengo, S.C., Amato, S.1995. Phyllocaulis variegatus (Mollusca: Veronicellidae) a new Intermediate Host for Brachylaima sp (Digenea: Brachylaimatidae). Mem Inst Oswaldo Cruz., 90: 621-622.

Thiengo, S.C., Faraco, A.F., Salgado, N.C., Cowie, R.H., Fernandez, M.A. 2007. Rapid spread of an invasive snail in South America: the giant African snail Achatina fulica. Biol Invasions., 9: 693-702.

Thiengo, S.C., Fernandez, M.A., Torres, E.J., Coelho, P.M., Lanfredi, R.M. 2008. First record of a nematode metastrongyloidea (Aelurostrongylus abstrusus larvae) in Achatina (Lissachatina) fulica (Mollusca, achatinidae) in brazilian. J. Invertebr. Pathol., 98, 34-39.

Thiengo, S. C., Maldonado, A., Mota, E. M., Torres, E.J., Caldeira, R,. Carvalho, O.S., Oliveira, A.P.M., Simões, R.O., Fernandez, M.A., Lanfredi. R. M. 2010. The giant African snail Achatina fulica as natural intermediate host of Angiostrongylus cantonensis in Pernambuco, northeast Brazil. Acta Trop., 115: 194-199.

Traversa, D., Di Cesare, A., Conboy, G. 2010. Canine and feline cardiopulmonary parasitic nematodes in Europe: emerging and underestimated. Parasit Vectors., 3: 1-22. 
Ubelaker, J.E, Hall, N.M. 1979. First report of Angiostrongylus costaricensis Morera and Céspedes, 1971 in the United States. J Parasitol., 65: 307.

Valente, R., Diaz, J.I., Salomón, O.D., Navone, G.T. 2016a. The role of Phyllocaulis variegatus (Mollusca: Veronicellidae) on the transmission of digenean parasites. Rev Mex Biodivers., 87: 255-257.

Valente, R., Diaz, J.I., Salomón, O.D., Navone, G.T. 2016b. Nematodes from Achatina fulica Bowdich, 1822 (Mollusca: Gastropoda) in Argentina. Helminthol., 53: 109112.

Valente R, Diaz, J.I., Salomon, O.D., Navone, G.T. 2017. Natural infection of the feline lungworm Aelurostrongylus abstrusus in the invasive snail Achatina fulica from Argentina. Vet Parasitol., 235: 17-19.

Valente, R., Robles, M.R., Navone, G.T., Diaz, J.I. 2018. Angiostrongylus spp. in the Americas: geographical and chronological distribution of definitive hosts versus disease reports. Mem Inst Oswaldo Cruz., 113: 1-10.

Vargas, M., Gómez-Pérez, J., Malek, E. 1992. First record of Angiostrongylus cantonensis (Chen 1935) (Nematoda: Metastrongylidae) in the Dominican Republic. Ann Trop Med Parasitol., 43: 253-255.

Velásquez-Trujillo, L.E. 2012. Cartilla: Diferencias entre el caracol invasor Achatina fulica y el caracol nativo Megalobulimus oblongus. Medellín: Unidad de Malacología Médica y Trematodos/Programa de Estudio y Control de Enfermedades Tropicales.

Virgillito, M. 2012. Panorama de los Gasterópodos terrestres exóticos en la Argentina (Gasterópoda Pulmonata Stylommatophora). Tesina de Licenciatura. Universidad de Buenos Aires.

Vogler, R.E., Beltramino, A.A., Sede, M.M., Gutiérrez Gregoric, D.E., Núñez, V., Rumi, A. 2013. The giant African snail, Achatina fulica (Gastropoda: Achatinidae): Using bioclimatic models to identify South American areas susceptible to invasion Amer Malac Bull. 31: 39-50.

Wallace, G., Rosen, L., 1969. Techniques for recovering and identifying larvae of Angiostrongylus cantonensis from molluscs. Malacol., 7, 427-438.

Wisnivesky, C. 2003. Ecología y epidemiología de las infecciones parasitarias. Libro Universitario Regional, Costa Rica.

Wittenberg, R., Cock, M.J. 2001. Especies Exóticas Invasoras: Una guía sobre las mejores prácticas de prevención y gestión. CABI Internacional, Wallingford, Oxon, Reino Unido: 1-5 pp. 
Yamaguti, S. 1975. A synoptical review of life histories of digenetic trematodes of vertebrates. Keigau Publishing, Tokyo.

Zanol, J., Fernandez, M.A., Martins de Oliveira, A.P. Moraes Russo, C.A., Thiengo, S.C. 2010. O caramujo exótico invasor Achatina fulica (Stylommatophora, Mollusca) no Estado do Rio de Janeiro (Brasil): situação actual. Biota Neotrop., 10: $447-451$.

Ziliotto, J.R., Kunzle, J.F., Fernandes, L., Prates-Campos, J.C., Britto-Costa, R. 1975: Angiostrongiliase: Apresentação de um provável caso. Rev Inst Med Trop S Paulo, 17: 312-318.

Zunino, M., Zullini, A. 2003. Biogeografía: la dimensión espacial de la evolución. Fondo de Cultura Económica, México.

Zuñiga, S., Cardona, V., Alvarado, D. 1983. Angiostrongilosis abdominal. Rev Med Hondur., 51:184-192. 